Becker, K., Sakai, H., et al., 1989

Proceedings of the Ocean Drilling Program, Scientific Results, Vol. 111

\title{
25. STABLE ISOTOPE HISTORY OF THE PLEISTOCENE AT ODP SITE $677^{1}$
}

\author{
N. J. Shackleton and M. A. Hall ${ }^{2}$
}

\begin{abstract}
Oxygen and carbon isotope ratio measurements are presented for Globigerinoides ruber and for benthic species (mainly Uvigerina spp.) in the Pleistocene and uppermost Pliocene section of ODP Hole 677A in the Panama Basin. This provides the best available continuous Pleistocene stable isotope records from any location, fully justifying the recoring of DSDP Site 504. Oxygen isotope stage 22 (age about $0.85 \mathrm{Ma}$ ) was of similar magnitude to the most extensive glacials of the Brunhes and constitutes a logical base for the middle Pleistocene. Oxygen isotope stages as defined by Ruddiman et al. (1986) and by Raymo et al. (in press) back to stage 104 are recognized. Although the internationally agreed base of the Quaternary at or near stage 62 (about $1.6 \mathrm{Ma}$ ) is not marked by a major isotopic event, it does approximate the base of a regime characterized by highly regular $41,000-y r$ climate cycles.

The records at Site 677 are ideal for time-series analyses and will permit a new attempt to develop a chronology for the early Pleistocene based on tuning to the orbital frequencies. The carbon isotope records also appear to contain considerable variance at orbital frequencies throughout the sequence analyzed.
\end{abstract}

\section{INTRODUCTION}

Deep Sea Drilling Project (DSDP) Site 504 (Shipboard Scientific Parties, 1983) was one of the first DSDP Sites to be cored using the then newly developed hydraulic piston corer (HPC) (Prell, Gardner, et al., 1982). Shackleton and Hall (1983) analyzed a large number of samples for oxygen isotope ratio using the planktonic species Globigerinoides ruber and indicated the important variability associated with the "Milankovitch frequencies," cycles with periods of about $22,000 \mathrm{yr}$ (precession), $41,000 \mathrm{yr}$ (tilt), and 100,000 yr (eccentricity) in the lower Pleistocene. However, the sequence recovered at Site 504 had serious limitations from the point of view of time-series analysis. In particular, the site was only cored once with a $4.5-\mathrm{m}$ corer, so there are numerous gaps in the recovered sequence. Because the accumulation rate is close to $4 \mathrm{~cm} / 1000 \mathrm{yr}$, the gaps are at an interval of roughly $100,000 \mathrm{yr}$, and because the distance between the bottom of one core and the top of the next is never known accurately, this made formal time-series analysis difficult.

Ocean Drilling Program (ODP) Leg 111 drilled at nearby Site 677 , permitting a new oxygen isotope record to be obtained. Holes $677 \mathrm{~A}\left(18^{\circ} 12.138^{\prime} \mathrm{N}, 83^{\circ} 44.220^{\prime} \mathrm{W}\right.$; $3461.2 \mathrm{~m}$ water depth) and $677 \mathrm{~B}\left(1^{\circ} 12.142^{\prime} \mathrm{N}, 83^{\circ} 44.220^{\prime} \mathrm{W} ; 3461.2 \mathrm{~m}\right.$ water depth) were cored with a depth offset intended to cover intercore gaps in the first hole with recovery in the second hole. Unfortunately the offset in depth was only about $1.5 \mathrm{~m}$, which is small compared with the uncertainty in the actual depth at which each core was recovered, seriously reducing the value of this exercise. Alexandrovich and Hays (this volume) have assessed the relative positions of cores in Holes 677A and 677B. At this time we have examined only a limited amount of material from Hole 677B.

\section{METHODS}

Samples were disaggregated by shaking in distilled water and washed through a $150-\mu \mathrm{m}$ sieve. The coarse fraction was dried and weighed. The fine fraction was settled for $24 \mathrm{hr}$, the water was siphoned off to about 1 $\mathrm{cm}$ above the sediment, and the residue dried in an oven at $50^{\circ} \mathrm{C}$ and weighed. Table 1 gives the percent coarse fraction based on these data. In a number of samples significant quantities of pyrite were noted in the

\footnotetext{
${ }^{1}$ Becker, K., Sakai, H., et al., 1989. Proc. ODP, Sci. Results, 111: College Station, TX (Ocean Drilling Program).

2 Godwin Laboratory for Quaternary Research, University of Cambridge, Free School Lane, Cambridge CB2 3RS, U.K.
}

coarse fraction; the weight of the $>150-\mu \mathrm{m}$ fraction may not always be dominated by foraminifers.

Globigerinoides ruber for isotopic analysis were picked from the $300-355-\mu \mathrm{m}$ fraction, and Uvigerina spp. or other benthic species were picked where possible from the $>250-\mu \mathrm{m}$ fraction. The selected specimens were crushed under analytical grade methanol and cleaned ultrasonically; further chemical cleaning was achieved using hydrogen peroxide instead of by vacuum roasting as was used by Shackleton and Hall (1983). In most sediments we were unable to detect any effect on isotopic composition depending on which of the two methods was used, but we have occasionally found cores in which foraminifers yielded an impure $\mathrm{CO}_{2}$ gas if vacuum roasted whereas the use of hydrogen peroxide appears to eliminate this problem. Stable isotope measurements were made using a VG Isogas 903 triple-collector mass spectrometer except for those run after October 1988 (samples A 88/2048 onward), which were analyzed on a VG Isogas SIRA 10 triple-collector mass spectrometer.

\section{RESULTS}

All isotopic measurements are tabulated in Tables 2 and 3, expressed as per mil deviations from the PDB standard (Epstein et al., 1953). Figure 1 shows oxygen isotope measurements as a function of adjusted (see "Correlations" section) depth in meters below seafloor. Oxygen isotope stages 1 to 23 (Emiliani, 1955; Shackleton and Opdyke, 1973) are easily recognized. Note that we have adopted the redefinition of stages 22 and 23 suggested by Ruddiman et al. (1986).

\section{Intercore Patches}

A necessary preliminary to developing a time series from an HPC-cored site is to assess the true extent of the depth relationship between the bottom of each core and the top of the next one. This is conveniently achieved by developing a composite depth section (Ruddiman et al., in press) in which a logically consistent set of depth adjustments is made to successively deeper cores in each of the two holes such that a continuous depth section can be constructed by patching together appropriate intervals from each hole. In the case of Site 677, Alexandrovich and Hays (this volume) have also taken account of data from DSDP Hole 504. We used their adjustments as a starting point and made additional small depth adjustments permitted by the fact that our sampling interval is $10 \mathrm{~cm}$, compared with their $50 \mathrm{~cm}$, so that we can make more accurate estimates of the intercore relationships where we have overlapping isotope data. Table 4 presents the adjustments to each core as given by Alexandrovich and Hays (this volume) and as here modified. 
Table 1. Percent Table 1 (continued). coarse fraction $(>150 \mu \mathrm{m})$ in Holes $677 \mathrm{~A}$ and $677 \mathrm{~B}$.

\begin{tabular}{|c|c|}
\hline $\begin{array}{l}\text { Depth }^{a} \\
\text { (mbsf) }\end{array}$ & $\begin{array}{c}>150-\mu \mathrm{m} \\
\text { fraction } \\
(\%)\end{array}$ \\
\hline \multicolumn{2}{|l|}{ Hole $677 \mathrm{~A}$} \\
\hline 0.40 & 13.22 \\
\hline 0.50 & 19.26 \\
\hline 0.60 & 16.91 \\
\hline 0.68 & 13.19 \\
\hline 0.80 & 10.40 \\
\hline 0.90 & 7.30 \\
\hline 0.98 & 10.74 \\
\hline 1.10 & 10.13 \\
\hline 1.20 & 9.84 \\
\hline 1.30 & 9.50 \\
\hline 1.40 & 11.78 \\
\hline 1.50 & 13.46 \\
\hline 1.60 & 9.78 \\
\hline 1.70 & 11.03 \\
\hline 1.80 & 10.29 \\
\hline 1.90 & 8.74 \\
\hline 2.00 & 7.50 \\
\hline 2.10 & 9.55 \\
\hline 2.20 & 14.29 \\
\hline 2.30 & 9.76 \\
\hline 2.40 & 8.77 \\
\hline 2.47 & 7.93 \\
\hline 2.58 & 6.35 \\
\hline 2.70 & 12.90 \\
\hline 2.80 & 8.89 \\
\hline 2.90 & 13.60 \\
\hline 3.00 & 14.78 \\
\hline 3.10 & 13.54 \\
\hline 3.20 & 10.69 \\
\hline 3.30 & 9.83 \\
\hline 3.40 & 5.92 \\
\hline 3.50 & 3.84 \\
\hline 3.60 & 5.56 \\
\hline 3.70 & 3.93 \\
\hline 3.80 & 3.29 \\
\hline 3.90 & 2.40 \\
\hline 3.97 & 2.48 \\
\hline 4.08 & 2.76 \\
\hline 4.20 & 3.23 \\
\hline 4.30 & 4.84 \\
\hline 4.60 & 3.58 \\
\hline 4.70 & 2.12 \\
\hline 4.80 & 3.71 \\
\hline 4.90 & 2.49 \\
\hline 4.98 & 3.06 \\
\hline 5.13 & 9.33 \\
\hline 5.20 & 7.30 \\
\hline 5.30 & 4.82 \\
\hline 5.40 & 6.78 \\
\hline 5.60 & 3.77 \\
\hline 5.70 & 4.76 \\
\hline 5.80 & 2.88 \\
\hline 5.90 & 4.44 \\
\hline 6.00 & 5.01 \\
\hline 6.10 & 6.25 \\
\hline 6.59 & 2.42 \\
\hline 6.68 & 1.89 \\
\hline 6.80 & 2.29 \\
\hline 6.92 & 1.07 \\
\hline 7.18 & 2.67 \\
\hline 7.29 & 1.82 \\
\hline 7.49 & 2.52 \\
\hline 7.59 & 2.75 \\
\hline 7.70 & 2.34 \\
\hline 7.80 & 2.58 \\
\hline 7.90 & 2.16 \\
\hline 8.01 & 1.10 \\
\hline 8.11 & 1.59 \\
\hline 8.20 & 2.17 \\
\hline 8.30 & 1.13 \\
\hline
\end{tabular}

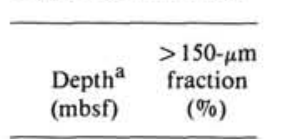

Hole 677A (Cont.)

$\begin{array}{ll}8.43 & 1.06 \\ 8.60 & 0.67 \\ 8.68 & 0.85 \\ 8.80 & 1.04 \\ 8.90 & 2.35\end{array}$

$\begin{array}{ll}8.90 & 2.35 \\ 8.99 & 3.54\end{array}$

$\begin{array}{ll}9.08 & 2.44\end{array}$

$9.20 \quad 2.12$

$\begin{array}{ll}9.29 & 2.00 \\ 9.39 & 3.01\end{array}$

$\begin{array}{ll}9.39 & 3.01 \\ 9.49 & 2.77\end{array}$

$\begin{array}{ll}9.59 & 2.40\end{array}$

$\begin{array}{ll}9.69 & 2.95 \\ 9.78 & 3.64\end{array}$

$\begin{array}{ll}9.92 & 1.84\end{array}$

$\begin{array}{ll}10.00 & 1.15 \\ 10.10 & 0.93\end{array}$

$\begin{array}{ll}10.10 & 0.93 \\ 10.18 & 1.34\end{array}$

$\begin{array}{ll}10.29 & 0.92\end{array}$

$\begin{array}{ll}10.39 & 1.11 \\ 10.49 & 1.41\end{array}$

$\begin{array}{ll}10.49 & 1.41 \\ 10.59 & 1.98\end{array}$

$10.70 \quad 2.15$

$\begin{array}{ll}10.77 & 2.60 \\ 10.89 & 1.74\end{array}$

$10.99 \quad 1.65$

$\begin{array}{ll}11.11 & 1.15 \\ 11.17 & 1.35\end{array}$

$\begin{array}{ll}11.17 & 1.35 \\ 11.29 & 1.11\end{array}$

$11.40 \quad 1.67$

$\begin{array}{ll}11.49 & 1.75 \\ 11.60 & 1.06\end{array}$

$\begin{array}{ll}11.60 & 1.06 \\ 11.68 & 1.46\end{array}$

$\begin{array}{ll}11.79 & 1.46 \\ 11.89 & 1.29\end{array}$

$\begin{array}{ll}11.89 & 1.29 \\ 11.99 & 1.23\end{array}$

$12.09 \quad 2.13$

$\begin{array}{ll}12.20 & 4.47 \\ 12.29 & 4.55\end{array}$

$\begin{array}{ll}12.29 & 4.55 \\ 12.39 & 3.15\end{array}$

12.49

12.59
12.69

$\begin{array}{ll}12.69 & 3.27\end{array}$

$12.79 \quad 3.28$

$\begin{array}{ll}12.92 & 2.88 \\ 13.01 & 2.94\end{array}$

$\begin{array}{ll}13.01 & 2.94 \\ 13.10 & 2.55\end{array}$

$13.18 \quad 2.74$

$13.30 \quad 3.66$

$\begin{array}{ll}13.40 & 2.93 \\ 13.52 & 2.25\end{array}$

13.60

$\begin{array}{ll}13.70 & 1.65 \\ 13.80 & 1.39\end{array}$

$\begin{array}{ll}13.80 & 1.39 \\ 13.89 & 3.15\end{array}$

14.00

14.10

14.20

14.42

14.50
14.60

14.60

$14.68 \quad 2.02$

$\begin{array}{ll}14.80 & 2.95\end{array}$

$14.89 \quad 2.21$

$14.99 \quad 3.68$

$15.09 \quad 2.05$

$\begin{array}{ll}15.20 & 3.25 \\ 15.29 & 3.87\end{array}$

$\begin{array}{ll}15.39 & 5.23\end{array}$

$15.49 \quad 5.40$

$\begin{array}{ll}15.49 & 5.40 \\ 15.59 & 5.06\end{array}$

15.68
Table 1 (continued).
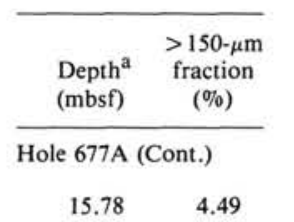

$\begin{array}{ll}15.78 & 4.49 \\ 15.80 & 3.73 \\ 15.90 & 2.96 \\ 16.00 & 4.36 \\ 16.10 & 3.02 \\ 16.20 & 3.51 \\ 16.30 & 2.61 \\ 16.37 & 2.85 \\ 16.50 & 3.48\end{array}$

$\begin{array}{ll}16.50 & 3.48 \\ 16.60 & 4.03\end{array}$

$16.68 \quad 2.69$

$16.80 \quad 2.97$

$\begin{array}{ll}16.86 & 3.12 \\ 16.90 & 2.42\end{array}$

$\begin{array}{ll}16.90 & 2.42 \\ 17.00 & 2.88\end{array}$

$17.10 \quad 1.38$

$\begin{array}{ll}17.20 & 1.93 \\ 17.30 & 1.63\end{array}$

$\begin{array}{ll}17.40 & 2.29\end{array}$

$\begin{array}{ll}17.50 & 1.78 \\ 17.60 & 1.78\end{array}$

$\begin{array}{ll}17.60 & 1.78 \\ 17.70 & 2.70\end{array}$

$17.80 \quad 2.51$

$\begin{array}{ll}17.87 & 2.96 \\ 18.00 & 3.73\end{array}$

$\begin{array}{ll}18.00 & 3.73 \\ 18.10 & 3.18\end{array}$

$18.18 \quad 3.47$

$\begin{array}{ll}18.30 & 3.99\end{array}$

$\begin{array}{ll}18.40 & 3.98\end{array}$

$18.50 \quad 4.59$

$18.60 \quad 3.06$

$\begin{array}{ll}18.70 & 2.69 \\ 18.80 & 2.97\end{array}$

$18.90 \quad 2.94$

$19.00 \quad 5.85$

$19.10 \quad 6.20$

$19.20 \quad 5.64$

19.29

19.37

19.48

19.59

19.67

19.79
19.90

19.90
20.00

20.10

20.20

20.30

20.40

20.50

20.60

20.70

20.80

20.87

21.00

21.09

21.20
21.30

21.30
21.70

21.80

21.90

22.00

22.10

22.20

22.30

22.37

22.50

22.60

22.67

22.80

23.00

23.10
5.52

5.05
5.29

3.60

2.44

3.14

2.86

2.66
1.80

1.48

1.35

1.68
3.07

1.62

1.14

1.35

2.10
4.22

2.35

1.86

2.47

1.63

2.05
1.54

1.51

2.63

2.60

3.58
6.42

7.23

4.65

3.47
4.56

4.41

4.92

4.21
Table 1 (continued).

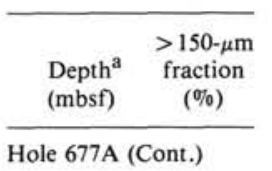

$\begin{array}{ll}23.20 & 4.69 \\ 23.30 & 3.30 \\ 23.40 & 4.61 \\ 23.50 & 4.53 \\ 23.60 & 4.30\end{array}$

$\begin{array}{ll}23.70 & 4.78\end{array}$

$\begin{array}{ll}23.80 & 1.64 \\ 23.87 & 2.68\end{array}$

$24.00 \quad 1.94$

$24.09 \quad 1.90$

$\begin{array}{ll}24.18 & 2.57\end{array}$

$\begin{array}{ll}24.29 & 1.77 \\ 24.39 & 1.72\end{array}$

$\begin{array}{ll}24.59 & 2.05\end{array}$

$24.70 \quad 2.24$

$24.80 \quad 1.73$

24.90

$25.00 \quad 1.35$

25.09

25.19

25.29
25.28

25.28
25.39

25.49

25.59
25.66

26.02

26.10
26.20

26.30

26.37

26.50
26.60

26.71
26.80

26.80
26.90

27.00

27.10
27.19

27.19
27.30

27.41

27.49

27.56
27.68

27.80

27.90

27.99

28.10

28.20
28.30

28.30
28.40

28.50

28.60
28.70

28.77

28.87

28.99

29.09

29.18

29.29
29.40

29.40
29.50

29.60

29.70
29.80

29.80
29.90

30.00

30.10

30.20

30.40

30.50

3.06
2.61
Table 1 (continued).

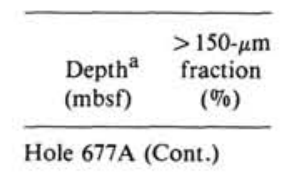

$$
30.59 \quad 3.10
$$

$\begin{array}{ll}30.59 & 3.10 \\ 30.68 & 2.38 \\ 30.80 & 4.65 \\ 30.90 & 5.41 \\ 31.00 & 5.17\end{array}$

$\begin{array}{ll}31.10 & 6.58\end{array}$

$\begin{array}{ll}31.20 & 10.31\end{array}$

$\begin{array}{ll}31.30 & 6.82\end{array}$

$\begin{array}{ll}31.50 & 4.47\end{array}$

$31.60 \quad 4.74$

$31.70 \quad 4.37$

$\begin{array}{ll}31.80 & 4.91 \\ 31.92 & 5.62\end{array}$

$\begin{array}{ll}31.92 & 5.62 \\ 32.00 & 4.21\end{array}$

$\begin{array}{ll}32.10 & 5.03\end{array}$

$\begin{array}{ll}32.18 & 3.38 \\ 32.30 & 2.50\end{array}$

$\begin{array}{ll}32.30 & 2.50 \\ 32.40 & 3.92\end{array}$

$\begin{array}{ll}32.50 & 5.37\end{array}$

$32.60 \quad 6.82$

32.70

32.80

32.90

33.00

33.10
33.30

33.40

33.40
33.60

33.60
33.68

33.68
33.80

33.80
33.90

34.00

34.10

34.80
34.90

34.90
35.00

35.10
35.50

35.50
35.60

35.60
35.70

35.90
36.00

36.00
36.10

36.21

36.30

36.40
36.50

36.50

36.60
36.70

36.70
36.80

36.87

37.00

37.10
37.18

37.18
37.28
37.40

37.40
37.50

37.50
37.60

37.71

37.80

37.90

38.00
38.10

38.10
38.20

38.31

38.42

38.50

38.60
38.68

38.68

38.77
38.90

5.79
6.87

7.86

5.67

3.16

3.10

2.06

2.40

1.28

1.55

1.37

1.27

3.41
4.29

2.73 
Table 1 (continued).

\begin{tabular}{lc}
\hline $\begin{array}{l}\text { Depth } \\
\text { (mbsf) }\end{array}$ & $\begin{array}{c}>150-\mu \mathrm{m} \\
\text { fraction } \\
(\%)\end{array}$ \\
\hline
\end{tabular}

Hole 677A (Cont.)

\begin{tabular}{|c|c|}
\hline 46.68 & 4.13 \\
\hline 46.80 & 2.78 \\
\hline 46.90 & 2.55 \\
\hline 47.00 & 2.70 \\
\hline 47.10 & 1.91 \\
\hline 47.21 & 5.01 \\
\hline 47.30 & 5.81 \\
\hline 47.40 & 5.98 \\
\hline 47.50 & 7.77 \\
\hline 47.60 & 4.27 \\
\hline 47.70 & 7.49 \\
\hline 47.81 & 7.96 \\
\hline 47.90 & 9.29 \\
\hline 48.00 & 6.79 \\
\hline 48.10 & 6.93 \\
\hline 48.20 & 5.78 \\
\hline 48.30 & 1.68 \\
\hline 48.80 & 2.02 \\
\hline 48.90 & 1.12 \\
\hline 49.00 & 1.19 \\
\hline 49.08 & 1.52 \\
\hline 49.19 & 1.88 \\
\hline 49.30 & 2.11 \\
\hline 49.39 & 2.56 \\
\hline 49.49 & 3.72 \\
\hline 49.59 & 4.96 \\
\hline 49.66 & 4.29 \\
\hline 49.80 & 3.43 \\
\hline 49.90 & 2.71 \\
\hline 50.00 & 2.76 \\
\hline 50.10 & 2.17 \\
\hline 50.21 & 2.40 \\
\hline 50.29 & 2.32 \\
\hline 50.40 & 1.69 \\
\hline 50.50 & 1.83 \\
\hline 50.60 & 1.36 \\
\hline 50.70 & 1.37 \\
\hline 50.80 & 1.85 \\
\hline 50.90 & 1.07 \\
\hline 50.99 & 2.49 \\
\hline 51.10 & 3.20 \\
\hline 51.17 & 3.70 \\
\hline 51.30 & 2.50 \\
\hline 51.40 & 2.86 \\
\hline 51.50 & 1.86 \\
\hline 51.60 & 2.52 \\
\hline 51.80 & 1.44 \\
\hline 51.90 & 2.05 \\
\hline 52.00 & 1.04 \\
\hline 52.10 & 1.21 \\
\hline 52.20 & 1.31 \\
\hline 52.30 & 0.90 \\
\hline 52.40 & 0.81 \\
\hline 52.50 & 1.74 \\
\hline 52.61 & 2.04 \\
\hline 52.67 & 1.89 \\
\hline 52.80 & 2.80 \\
\hline 52.90 & 4.16 \\
\hline 53.00 & 7.09 \\
\hline 53.10 & 3.85 \\
\hline 53.30 & 4.76 \\
\hline 53.40 & 2.39 \\
\hline 53.48 & 2.61 \\
\hline 53.60 & 1.76 \\
\hline 53.70 & 1.27 \\
\hline 53.80 & 1.98 \\
\hline 53.80 & 2.57 \\
\hline 53.90 & 2.60 \\
\hline 54.00 & 3.05 \\
\hline 54.10 & 5.55 \\
\hline 54.20 & 4.64 \\
\hline 54.30 & 4.59 \\
\hline 54.40 & 5.88 \\
\hline
\end{tabular}

Table 1 (continued).

\begin{tabular}{cc}
\hline $\begin{array}{l}\text { Depth } \\
\text { (mbsf) }\end{array}$ & $\begin{array}{c}>150-\mu \mathrm{m} \\
\text { fraction } \\
(\%)\end{array}$ \\
Hole 677A (Cont.)
\end{tabular}

Table 1 (continued).

\begin{tabular}{cc}
$\begin{array}{l}\text { Depth } \\
(m b s)\end{array}$ & $\begin{array}{c}>150-\mu \mathrm{m} \\
\text { fraction } \\
(\%)\end{array}$ \\
\hline Hole 677A (Cont.)
\end{tabular}

Table 1 (continued).

\begin{tabular}{cc}
$\begin{array}{c}\text { Depth } \\
\text { (mbsf) }\end{array}$ & $\begin{array}{c}>150-\mu \mathrm{m} \\
\text { fraction } \\
(\%)\end{array}$ \\
\hline Hole 677A (Cont.)
\end{tabular}

$\begin{array}{ll}54.50 & 4.60 \\ 54.60 & 4.29 \\ 54.68 & 5.20 \\ 54.80 & 5.62 \\ 54.90 & 5.59 \\ 55.00 & 4.25 \\ 55.10 & 3.34 \\ 55.20 & 4.01 \\ 55.30 & 4.68 \\ 55.40 & 3.06 \\ 55.50 & 2.78 \\ 55.60 & 1.86 \\ 55.70 & 2.68 \\ 55.80 & 2.66\end{array}$

$\begin{array}{ll}61.80 & 1.23 \\ 61.90 & 1.50 \\ 62.00 & 1.71 \\ 62.10 & 1.76 \\ 62.18 & 2.04 \\ 62.30 & 1.08 \\ 62.40 & 1.53 \\ 62.50 & 1.35 \\ 62.60 & 1.80 \\ 62.70 & 2.47 \\ 62.80 & 1.50 \\ 62.90 & 2.65 \\ 63.00 & 1.59 \\ 63.10 & 1.97 \\ 63.18 & 1.44 \\ 63.30 & 2.10 \\ 63.40 & 1.77\end{array}$

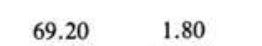

$\begin{array}{ll}69.20 & 1.80 \\ 69.30 & 0.56 \\ 69.40 & 0.78 \\ 69.50 & 1.19 \\ 69.60 & 1.71\end{array}$

Table 1 (continued).

Table 1 (continued).

\begin{tabular}{cc}
$\begin{array}{c}\text { Depth } \\
(m b s)\end{array}$ & $\begin{array}{c}>150-\mu \mathrm{m} \\
\text { fraction } \\
(\%)\end{array}$ \\
\hline Hole 677A (Cont.)
\end{tabular}

\begin{tabular}{cc}
$\begin{array}{l}\text { Depth } \\
\text { (mbsf) }\end{array}$ & $\begin{array}{c}>150-\mu \mathrm{m} \\
\text { fraction } \\
(\%)\end{array}$ \\
\hline Hole 677A (Cont.)
\end{tabular}

76.60
76.70
76.81
77.20
77.30
77.39
77.50
77.60
77.70
77.78
77.90
78.00
78.10
78.18
78.30
78.40
78.50

1.03
1.48
0.60

$\begin{array}{ll}84.68 & 1.10 \\ 84.79 & 1.27\end{array}$

$\begin{array}{ll}84.79 & 1.27 \\ 84.89 & 1.56\end{array}$

$\begin{array}{ll}85.00 & 1.56 \\ 85.10 & 2.10\end{array}$

$\begin{array}{llllll}69.60 & 1.71 & 3.01 & 85.10 & 2.76 \\ 69.70 & 2.52 & 77.39 & 2.39 & 85.20 & 3.82\end{array}$

$\begin{array}{llllll}69.80 & 1.55 & 77.50 & 2.18 & 85.30 & 3.11\end{array}$

$\begin{array}{llllll}69.90 & 1.06 & 77.60 & 2.92 & 85.39 & 3.51\end{array}$

$\begin{array}{llllll}70.00 & 1.53 & 77.70 & 1.72 & 85.50 & 3.83 \\ 70.10 & 0.87 & 77.78 & 2.17 & 85.60 & 1.36\end{array}$

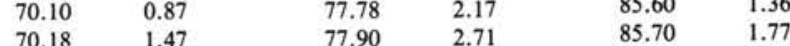

$\begin{array}{llllll}70.18 & 1.47 & 77.90 & 2.71 & 85.70 & 1.77 \\ 70.30 & 0.92 & 78.00 & 2.22 & 85.79 & 0.78 \\ 70.40 & 1.75 & 78.10 & 2.70 & 85.90 & 1.09\end{array}$

$\begin{array}{llllll}70.40 & 1.75 & 78.10 & 2.70 & 85.90 & 1.09 \\ 70.50 & 3.25 & 78.18 & 1.99 & 86.00 & 3.56\end{array}$

\begin{tabular}{llllll}
70.50 & 3.25 & 78.18 & 1.99 & 86.00 & 3.56 \\
70.60 & 2.66 & 78.30 & 2.78 & 86.10 & 2.25 \\
\hline
\end{tabular}

$\begin{array}{llllll}70.70 & 2.41 & 78.40 & 2.06 & 86.18 & 2.50 \\ 70.80 & 2.37 & 78.50 & 1.79 & 86.30 & 1.46\end{array}$

$\begin{array}{llllll}70.80 & 2.37 & 78.50 & 1.79 & 86.30 & 1.46 \\ 70.90 & 1.19 & 78.60 & 2.16 & 86.40 & 1.14\end{array}$

$\begin{array}{llllll}70.90 & 1.19 & 78.60 & 2.16 & 86.40 & 1.14 \\ 71.00 & 1.06 & 78.70 & 2.30 & 86.50 & 0.98\end{array}$

$\begin{array}{llllll}71.10 & 1.45 & 78.80 & 2.14 & 86.60 & 0.43 \\ 71.20 & 1.18 & 78.89 & 1.70 & 86.70 & 1.49\end{array}$

$\begin{array}{llllll}71.20 & 1.18 & 78.89 & 1.70 & 86.70 & 1.49 \\ 71.30 & 0.81 & 79.00 & 2.63 & 86.80 & 1.60\end{array}$

\begin{tabular}{llllll}
71.30 & 0.81 & 79.00 & 2.63 & 86.80 & 1.60 \\
71.40 & 1.70 & 79.10 & 2.89 & 86.89 & 2.41 \\
\hline
\end{tabular}

$\begin{array}{llllll}71.50 & 1.71 & 79.20 & 2.06 & 87.00 & 1.25 \\ 71.60 & 1.48 & 79.30 & 1.71 & 87.10 & 1.60\end{array}$

$\begin{array}{llllll}71.60 & 1.48 & 79.30 & 1.71 & 87.10 & 1.60 \\ 71.68 & 2.45 & 79.40 & 1.47 & 87.20 & 0.83\end{array}$

$\begin{array}{llllll}71.80 & 2.05 & 79.50 & 1.92 & 87.29 & 1.84 \\ 71.90 & 0.78 & 79.60 & 1.55 & 87.39 & 1.55\end{array}$

$\begin{array}{llllll}71.90 & 0.78 & 79.60 & 1.55 & 87.39 & 1.55 \\ 72.00 & 1.22 & 79.68 & 2.45 & 87.50 & 1.50\end{array}$

$\begin{array}{llllll}72.00 & 1.22 & 79.68 & 2.45 & 87.50 & 1.50 \\ 72.10 & 0.46 & 79.80 & 1.86 & 87.60 & 0.55\end{array}$

$\begin{array}{llllll}72.10 & 0.46 & 79.80 & 1.86 & 87.60 & 0.55 \\ 72.20 & 0.79 & 79.90 & 1.68 & 87.68 & 0.86 \\ 72.30 & 1.17 & 80.01 & 1.53 & 87.80 & 1.68\end{array}$

$\begin{array}{llllll}72.20 & 0.79 & 79.90 & 1.68 & 87.80 & 1.68 \\ 72.30 & 1.17 & 80.01 & 1.53 & 87.80\end{array}$

$\begin{array}{llllll}72.30 & 1.17 & 80.01 & 1.53 & 87.80 & 1.68 \\ 72.40 & 1.32 & 80.10 & 2.53 & 87.90 & 1.34 \\ 72.50 & 1.31 & 80.20 & 1.62 & 88.00 & 1.18\end{array}$

$\begin{array}{llllll}72.50 & 1.31 & 80.20 & 1.62 & 88.00 & 1.18 \\ 72.70 & 1.62 & 80.29 & 2.12 & 88.10 & 1.35\end{array}$

$\begin{array}{llllll}72.70 & 1.62 & 80.29 & 2.12 & 88.10 & 1.35 \\ 72.80 & 1.03 & 80.39 & 1.83 & 88.20 & 0.89\end{array}$

$\begin{array}{llllll}72.80 & 1.03 & 80.39 & 1.83 & 88.20 & 0.89 \\ 72.89 & 0.99 & 80.49 & 2.63 & 88.30 & 1.45 \\ 73.00 & 0.85 & 80.59 & 2.12 & 88.39 & 2.55\end{array}$

$\begin{array}{llllll}73.00 & 0.85 & 80.59 & 2.12 & 88.39 & 2.55\end{array}$

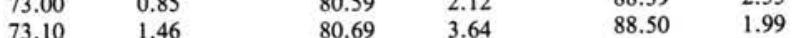

$\begin{array}{llllll}73.10 & 1.46 & 80.69 & 3.64 & 88.50 & 1.99 \\ 73.20 & 1.84 & 80.79 & 2.60 & 88.60 & 1.70\end{array}$

$\begin{array}{llllll}73.28 & 1.71 & 80.90 & 3.46 & 88.70 & 1.42 \\ 73.40 & 2.29 & 81.00 & 4.14 & 88.79 & 1.65\end{array}$

$\begin{array}{llllll}73.40 & 2.29 & 81.00 & 4.14 & 88.79 & 1.65 \\ 73.49 & 2.71 & 81.10 & 3.52 & 88.90 & 2.18\end{array}$

$\begin{array}{llllll}73.49 & 2.71 & 81.10 & 3.52 & 88.90 & 2.18 \\ 73.59 & 2.78 & 81.18 & 3.47 & 89.00 & 2.03\end{array}$

$\begin{array}{llllll}73.68 & 2.68 & 81.30 & 4.50 & 89.10 & 2.23\end{array}$

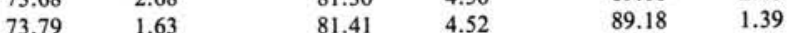

$\begin{array}{llllll}73.79 & 1.63 & 81.41 & 4.52 & 89.18 & 1.39 \\ 73.89 & 1.21 & 81.50 & 4.11 & 89.30 & 1.66\end{array}$

$\begin{array}{llllll}74.00 & 1.25 & 81.60 & 5.29 & 89.40 & 2.28 \\ 74.09 & 1.58 & 82.20 & 3.24 & 89.50 & 1.90\end{array}$

$\begin{array}{llllll}74.09 & 1.58 & 82.20 & 3.24 & 89.50 & 1.90\end{array}$

$\begin{array}{llllll}74.20 & 1.73 & 82.30 & 2.88 & 89.60 & 1.12 \\ 74.29 & 1.68 & 82.39 & 2.31 & 89.70 & 1.07\end{array}$

$\begin{array}{llllll}74.29 & 1.68 & 82.39 & 2.31 & 89.70 & 1.07 \\ 74.37 & 1.84 & 82.50 & 2.80 & 89.80 & 1.59\end{array}$

$\begin{array}{llllll}74.37 & 1.84 & 82.50 & 2.80 & 89.80 & 1.59 \\ 74.48 & 1.09 & 82.60 & 1.43 & 89.89 & 1.55\end{array}$

$\begin{array}{llllll}74.48 & 1.09 & 82.60 & 1.43 & 89.89 & 1.55 \\ 74.59 & 1.22 & 82.70 & 1.02 & 90.00 & 2.80\end{array}$

$\begin{array}{llllll}74.69 & 0.89 & 82.80 & 2.00 & 90.10 & 0.91\end{array}$

$\begin{array}{llllll}74.78 & 1.40 & 82.90 & 0.39 & 90.20 & 0.89\end{array}$

$\begin{array}{llllll}74.78 & 1.40 & 82.90 & 0.39 & 9.29 & 1.49\end{array}$

$\begin{array}{llllll}74.99 & 1.33 & 83.10 & 0.60 & 90.40 & 1.66\end{array}$

$\begin{array}{llllll}75.09 & 2.28 & 83.18 & 0.81 & 90.50 & 0.87\end{array}$

$\begin{array}{llllll}75.18 & 1.64 & 83.30 & 0.96 & 90.60 & 1.60 \\ 75.30 & 2.63 & 83.40 & 0.73 & 90.67 & 1.93\end{array}$

$\begin{array}{llllll}75.30 & 2.63 & 83.40 & 0.73 & 90.67 & 1.93 \\ 75.40 & 2.09 & 83.50 & 0.89 & 90.80 & 2.75\end{array}$

$\begin{array}{llllll}75.50 & 2.68 & 83.60 & 1.08 & 90.90 & 1.69\end{array}$

$\begin{array}{llllll}75.50 & 2.68 & 83.60 & 1.08 & 91.00 & 1.35\end{array}$

$\begin{array}{llllll}75.70 & 2.08 & 83.70 & 2.28 & 91.10 & 2.01\end{array}$

$\begin{array}{llllll}75.80 & 0.97 & 83.89 & 0.67 & 91.20 & 3.02 \\ 75.89 & 1.31 & 84.00 & 0.64 & 91.30 & 2.53\end{array}$

$\begin{array}{llllll}75.89 & 1.31 & 84.00 & 0.64 & 91.30 & 2.53 \\ 76.00 & 1.09 & 84.10 & 0.57 & 91.39 & 1.65\end{array}$

$\begin{array}{llllll}76.00 & 1.09 & 84.10 & 0.57 & 91.39 & 1.65 \\ 76.10 & 0.40 & 84.20 & 1.03 & 91.50 & 1.38\end{array}$

$\begin{array}{llllll}76.20 & 1.34 & 84.29 & 1.48 & 91.60 & 1.90\end{array}$

$\begin{array}{llllll}76.20 & 1.34 & 84.29 & 1.48 & 91.60 & 1.90 \\ 76.27 & 1.92 & 84.40 & 1.12 & 91.70 & 0.99\end{array}$

$\begin{array}{llllll}76.27 & 1.92 & 84.40 & 1.12 & 91.70 & 0.99 \\ 76.40 & 0.86 & 84.50 & 1.10 & 91.72 & 1.97 \\ 76.50 & 1.02 & 84.60 & 0.88 & 91.80 & 1.73\end{array}$ 
Table 1 (continued).

\begin{tabular}{lc}
\hline $\begin{array}{l}\text { Depth } \\
\text { (mbsf) }\end{array}$ & $\begin{array}{c}>150-\mu \mathrm{m} \\
\text { fraction } \\
(\%)\end{array}$ \\
\hline
\end{tabular}

Hole 677A (Cont.)

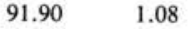

$92.00 \quad 1.08$

$\begin{array}{ll}92.10 & 1.67 \\ 92.20 & 1.29\end{array}$

$92.20 \quad 1.86$

$92.30 \quad 1.61$

$\begin{array}{ll}92.40 & 2.55\end{array}$

$92.50 \quad 2.58$

$92.60 \quad 2.80$

$92.68 \quad 2.56$

$92.80 \quad 2.76$

$92.90 \quad 2.08$

$\begin{array}{ll}93.00 & 1.85 \\ 93.10 & 1.69\end{array}$

$93.20 \quad 2.55$

$93.30 \quad 2.97$

$\begin{array}{ll}93.40 & 1.67\end{array}$

$93.50 \quad 2.23$

$93.60 \quad 4.19$

$93.70 \quad 3.10$

$93.80 \quad 1.92$

$93.90 \quad 1.42$

$94.00 \quad 1.70$

$94.10 \quad 1.89$

$\begin{array}{ll}94.18 & 2.77\end{array}$

$94.30 \quad 3.32$

$94.40 \quad 1.83$

$\begin{array}{ll}94.50 & 3.28\end{array}$

$94.60 \quad 3.35$

$94.70 \quad 2.27$

$94.80 \quad 1.90$

$94.90 \quad 1.60$

$\begin{array}{ll}95.00 & 1.87 \\ 95.10 & 2.56\end{array}$

$\begin{array}{ll}95.10 & 2.56 \\ 95.20 & 2.28\end{array}$

$95.30 \quad 1.92$

$95.40 \quad 1.46$

$95.50 \quad 1.80$

$95.60 \quad 1.99$

$\begin{array}{ll}95.68 & 2.27\end{array}$

$95.80 \quad 2.25$

$95.90 \quad 2.42$

$96.00 \quad 2.55$

$96.10 \quad 2.66$

$96.20 \quad 2.67$

$96.30 \quad 2.31$

$96.40 \quad 2.82$

$96.50 \quad 1.80$

$96.60 \quad 2.35$

$96.70 \quad 1.63$

$96.80 \quad 2.68$

$96.90 \quad 2.65$

$97.00 \quad 2.01$

$97.10 \quad 2.29$

$97.18 \quad 2.56$

$97.30 \quad 1.96$

$97.40 \quad 1.28$

$97.50 \quad 1.92$

$97.60 \quad 2.62$

$\begin{array}{ll}97.70 & 2.73\end{array}$

$\begin{array}{ll}97.80 & 3.50 \\ 97.90 & 3.49\end{array}$

$98.00 \quad 4.22$

$98.10 \quad 3.29$

$98.20 \quad 2.62$

$98.30 \quad 1.27$

$98.41 \quad 1.39$

$98.50 \quad 0.79$

$98.61 \quad 1.36$

$98.68 \quad 2.00$

$98.80 \quad 2.23$

$98.90 \quad 2.08$

$99.00 \quad 1.51$

$99.10 \quad 1.24$
Table 1 (continued).

Table 1 (continued).

Table 1 (continued).

\begin{tabular}{cc}
$\begin{array}{l}\text { Depth } \\
\text { (mbsf) }\end{array}$ & $\begin{array}{c}>150-\mu \mathrm{m} \\
\text { fraction } \\
(\%)\end{array}$ \\
\hline Hole 677A (Cont.)
\end{tabular}

Hole 677A (Cont.)

$\begin{array}{ll}99.20 & 1.95 \\ 99.30 & 1.28 \\ 99.40 & 2.88 \\ 99.50 & 1.80 \\ 99.60 & 2.80 \\ 99.70 & 2.31 \\ 99.80 & 2.89\end{array}$

$\begin{array}{ll}99.80 & 2.89\end{array}$

$99.91 \quad 2.28$

$100.00 \quad 3.39$

$\begin{array}{ll}100.11 & 3.08 \\ 100.18 & 3.56\end{array}$

$\begin{array}{ll}100.30 & 2.01\end{array}$

$100.40 \quad 3.10$

$100.50 \quad 2.69$

$100.60 \quad 3.28$

$100.70 \quad 4.01$

$100.80 \quad 6.14$

$100.90 \quad 4.25$

$101.00 \quad 4.29$

$101.10 \quad 1.93$

$101.20 \quad 2.26$

$101.30 \quad 2.06$

$101.20 \quad 2.82$

$101.30 \quad 4.07$

$101.40 \quad 3.67$

$\begin{array}{ll}101.50 & 4.56\end{array}$

$101.60 \quad 3.33$

$101.70 \quad 2.59$

$\begin{array}{ll}101.80 & 2.59\end{array}$

$101.91 \quad 1.93$

$102.00 \quad 1.46$

$\begin{array}{ll}102.10 & 1.37\end{array}$

$102.18 \quad 0.62$

$102.30 \quad 0.47$

$102.40 \quad 1.36$

$102.50 \quad 1.21$

$102.60 \quad 1.06$

$102.70 \quad 0.79$

$102.80 \quad 0.84$

$\begin{array}{ll}102.90 & 0.77\end{array}$

$103.00 \quad 0.72$

$103.10 \quad 0.69$

$103.20 \quad 1.22$

$103.30 \quad 0.63$

$103.38 \quad 1.63$

$103.50 \quad 1.13$

$103.60 \quad 1.17$

$\begin{array}{ll}103.68 & 1.09\end{array}$

$\begin{array}{ll}103.80 & 1.09\end{array}$

$103.90 \quad 0.97$

$104.00 \quad 1.42$

$104.10 \quad 1.28$

$104.20 \quad 1.27$

$104.30 \quad 1.41$

$104.40 \quad 1.09$

$104.49 \quad 0.96$

$\begin{array}{ll}104.60 & 0.78\end{array}$

$104.70 \quad 0.82$

$\begin{array}{ll}104.80 & 1.07\end{array}$

$104.87 \quad 1.10$

$105.00 \quad 1.07$

$105.10 \quad 2.37$

$105.18 \quad 1.43$

$105.30 \quad 1.67$

$105.40 \quad 1.32$

$105.50 \quad 1.34$

$105.60 \quad 1.03$

$105.70 \quad 1.47$

$105.80 \quad 0.56$

$105.90 \quad 0.81$

$106.00 \quad 0.96$

$\begin{array}{ll}106.10 & 1.41 \\ 106.20 & 1.19\end{array}$

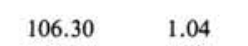

$\begin{array}{ll}106.30 & 1.04\end{array}$

$\begin{array}{ll}106.50 & 1.32 \\ 106.60 & 0.92\end{array}$

$106.60 \quad 1.41$

$106.68 \quad 0.88$

$\begin{array}{ll}106.79 & 0.90\end{array}$

$\begin{array}{ll}107.20 & 1.53\end{array}$

$\begin{array}{ll}107.30 & 2.07\end{array}$

$107.40 \quad 2.41$

$\begin{array}{ll}107.50 & 0.63\end{array}$

$\begin{array}{ll}107.60 & 1.39\end{array}$

$107.70 \quad 2.11$

$\begin{array}{ll}107.80 & 1.97\end{array}$

$\begin{array}{ll}107.87 & 1.32\end{array}$

$108.00 \quad 1.30$

$108.10 \quad 1.13$

$108.18 \quad 1.06$

$\begin{array}{ll}108.30 & 1.07\end{array}$

$\begin{array}{ll}108.40 & 1.09\end{array}$

$\begin{array}{ll}108.50 & 1.74\end{array}$

$108.60 \quad 1.71$

$\begin{array}{ll}108.70 & 1.01 \\ 108.80 & 2.07\end{array}$

108.90

$109.00 \quad 1.04$

$109.10 \quad 1.05$

$109.20 \quad 1.12$

$109.30 \quad 1.11$

$109.37 \quad 1.64$

$109.50 \quad 1.31$

$109.60 \quad 1.03$

$\begin{array}{ll}109.68 & 1.07 \\ 109.80 & 1.07\end{array}$

$109.90 \quad 1.1$

$110.00 \quad 1.42$

$110.10 \quad 0.93$

$110.20 \quad 1.37$

$110.30 \quad 1.02$

$110.40 \quad 1.88$

$110.50 \quad 0.63$

$110.60 \quad 0.43$

$\begin{array}{ll}110.70 & 0.83\end{array}$

$110.80 \quad 1.34$

$110.71 \quad 0.65$

$110.81 \quad 0.79$

$\begin{array}{ll}110.90 & 0.99\end{array}$

$\begin{array}{ll}111.00 & 0.72\end{array}$

$111.10 \quad 0.99$

$111.20 \quad 1.41$

$111.30 \quad 0.95$

$111.42 \quad 1.16$

$111.50 \quad 1.02$

$111.60 \quad 0.85$

$\begin{array}{ll}111.68 & 0.97\end{array}$

$111.80 \quad 1.08$

$111.90 \quad 1.23$

$112.00 \quad 1.03$

$112.10 \quad 0.79$

$112.20 \quad 1.14$

$112.30 \quad 1.40$

$\begin{array}{ll}112.40 & 1.48\end{array}$

$112.50 \quad 1.10$

$112.60 \quad 1.93$

$112.70 \quad 0.90$

$112.80 \quad 1.36$

$112.92 \quad 2.33$

$113.00 \quad 1.38$

$113.10 \quad 1.56$

$113.17 \quad 1.49$

$113.30 \quad 1.15$

$113.40 \quad 1.06$

$\begin{array}{ll}113.70 & 1.30 \\ 113.80 & 1.51\end{array}$

\begin{tabular}{|c|c|}
\hline $\begin{array}{l}\text { Depth }^{\mathrm{a}} \\
\text { (mbsf) }\end{array}$ & $\begin{array}{c}>150-\mu \mathrm{m} \\
\text { fraction } \\
(\%)\end{array}$ \\
\hline
\end{tabular}

Hole 677A (Cont.)

113.90

114.00

114.10

114.20

114.30

114.42

114.50

114.60

114.68

114.80

114.90

115.10

115.20

115.30

115.40

115.50

115.60

115.70

115.80

115.91

116.00

116.10

116.20

116.30

116.40

116.50

116.55

116.71

116.80

116.90

117.00

117.10

117.20

117.29

117.42

117.50

117.61
117.68

117.80

117.90

118.00

118.10

118.20

118.30

118.40

118.50

118.60

118.70

118.80

118.92

119.00

119.10

119.18

119.30
119.40

119.50

119.60

119.71

119.80

119.90

120.00

120.10

120.20

120.30

120.40

120.50

120.60

120.70

120.80

120.91

121.00

121.10
121.18

1.73
1.89

1.89
1.30

1.30

1.02

1.89
1.11 
Table 2. Oxygen and carbon isotope data for Globigerinoides ruber in the 300-355- $\mu \mathrm{m}$ fraction in Holes 677 A and 677B.

\begin{tabular}{cccc}
\hline $\begin{array}{c}\text { Sample } \\
\text { code }\end{array}$ & $\begin{array}{l}\text { Depth }^{\mathrm{a}} \\
\text { (mbsf) }\end{array}$ & $\delta^{18} \mathrm{O}$ & $\delta^{13} \mathrm{C}$ \\
\hline
\end{tabular}

Hole 677A

\begin{tabular}{|c|c|c|c|}
\hline A $87 / 1004$ & 0.40 & -2.22 & 1.54 \\
\hline A $87 / 1005$ & 0.50 & -1.73 & 1.37 \\
\hline A $87 / 1006$ & 0.60 & -1.16 & 1.42 \\
\hline A $87 / 1007$ & 0.68 & -1.23 & 1.30 \\
\hline A $87 / 1274$ & 0.80 & -0.89 & 1.59 \\
\hline A $87 / 1340$ & 0.80 & -1.12 & 1.64 \\
\hline A $87 / 1253$ & 0.90 & -0.87 & 1.15 \\
\hline A $87 / 1010$ & 0.98 & -0.69 & 1.05 \\
\hline A $87 / 1227$ & 1.10 & -0.45 & 1.55 \\
\hline A $87 / 998$ & 1.20 & -0.66 & 1.09 \\
\hline A $87 / 999$ & 1.30 & -0.46 & 1.17 \\
\hline A $87 / 1000$ & 1.40 & -0.63 & 1.06 \\
\hline A $87 / 1226$ & 1.50 & -0.73 & 1.22 \\
\hline A $87 / 1225$ & 1.60 & -0.77 & 1.39 \\
\hline A $87 / 1224$ & 1.70 & -0.84 & 1.48 \\
\hline A $87 / 1223$ & 1.80 & -0.62 & 1.79 \\
\hline A $87 / 1222$ & 1.90 & -0.97 & 1.48 \\
\hline A $87 / 1259$ & 2.00 & -1.25 & 1.59 \\
\hline A $88 / 868$ & 2.00 & -1.21 & 1.05 \\
\hline A $87 / 1251$ & 2.10 & -0.98 & 1.17 \\
\hline A $88 / 869$ & 2.10 & -1.05 & 1.21 \\
\hline A $87 / 1209$ & 2.20 & -1.05 & 1.49 \\
\hline A $88 / 870$ & 2.20 & -1.10 & 1.07 \\
\hline A $87 / 1208$ & 2.30 & -1.29 & 1.40 \\
\hline A $87 / 1207$ & 2.40 & -1.26 & 1.33 \\
\hline A $87 / 1206$ & 2.47 & -1.12 & 1.37 \\
\hline A $87 / 1205$ & 2.58 & -1.24 & 1.00 \\
\hline A $87 / 1204$ & 2.70 & -1.17 & 1.34 \\
\hline A $87 / 1203$ & 2.80 & -1.35 & 1.17 \\
\hline A $87 / 1202$ & 2.90 & -1.16 & 1.41 \\
\hline A $87 / 1201$ & 3.03 & -1.55 & 1.23 \\
\hline A $87 / 1200$ & 3.10 & -1.41 & 1.22 \\
\hline A $87 / 1199$ & 3.20 & -1.52 & 1.20 \\
\hline A $87 / 1198$ & 3.30 & -1.47 & 1.19 \\
\hline A $87 / 1197$ & 3.40 & -1.48 & 1.84 \\
\hline A $87 / 1196$ & 3.50 & -1.13 & 1.33 \\
\hline A $87 / 1195$ & 3.60 & -1.22 & 1.40 \\
\hline A $87 / 1194$ & 3.70 & -1.21 & 0.94 \\
\hline A $87 / 1193$ & 3.80 & -1.20 & 1.17 \\
\hline A $87 / 1192$ & 3.90 & -1.29 & 1.43 \\
\hline A $87 / 1191$ & 3.97 & -1.06 & 1.54 \\
\hline A $87 / 1190$ & 4.08 & -1.37 & 1.94 \\
\hline A $87 / 1189$ & 4.20 & -1.80 & 1.73 \\
\hline A $87 / 1188$ & 4.30 & -1.76 & 1.71 \\
\hline A $87 / 1187$ & 4.60 & -1.72 & 1.87 \\
\hline A $87 / 1186$ & 4.70 & -1.91 & 1.68 \\
\hline A $88 / 844$ & 4.70 & -1.91 & 1.33 \\
\hline A $87 / 1185$ & 4.80 & -1.56 & 1.70 \\
\hline A $88 / 843$ & 4.80 & -1.47 & 1.58 \\
\hline A $87 / 1184$ & 4.90 & -1.40 & 1.67 \\
\hline A $88 / 842$ & 4.90 & -1.44 & 1.41 \\
\hline A $87 / 1183$ & 4.98 & -1.60 & 1.48 \\
\hline A $88 / 841$ & 4.98 & -1.66 & 1.28 \\
\hline A $87 / 1182$ & 5.13 & -1.64 & 1.58 \\
\hline A $88 / 840$ & 5.13 & -1.53 & 1.56 \\
\hline A $87 / 1181$ & 5.20 & -1.89 & 1.78 \\
\hline A $88 / 839$ & 5.20 & -1.85 & 1.49 \\
\hline A $87 / 1228$ & 5.30 & -1.93 & 1.78 \\
\hline A $88 / 838$ & 5.30 & -1.91 & 1.45 \\
\hline A $87 / 1229$ & 5.40 & -2.00 & 1.59 \\
\hline A $88 / 837$ & 5.40 & -1.85 & 1.38 \\
\hline A $87 / 1230$ & 5.60 & -1.76 & 1.66 \\
\hline A $88 / 836$ & 5.60 & -1.81 & 1.31 \\
\hline A $87 / 1254$ & 5.70 & -2.31 & ${ }^{b} 0.88$ \\
\hline A $87 / 1275$ & 5.70 & -2.01 & 1.35 \\
\hline A $87 / 1341$ & 5.70 & -2.00 & 1.34 \\
\hline A $88 / 835$ & 5.70 & -1.86 & 1.21 \\
\hline A $87 / 1255$ & 5.80 & -1.94 & $b_{1.19}$ \\
\hline A $88 / 834$ & 5.80 & -1.77 & 1.51 \\
\hline 'A $87 / 1276$ & 5.90 & -2.69 & ${ }^{b} 1.18$ \\
\hline A $88 / 833$ & 5.90 & -1.89 & 1.37 \\
\hline A $87 / 1257$ & 6.00 & -2.00 & ${ }^{b} 1.39$ \\
\hline A $88 / 832$ & 6.00 & -2.11 & 1.27 \\
\hline
\end{tabular}

Table 2 (continued).

\begin{tabular}{cccc}
\hline $\begin{array}{c}\text { Sample } \\
\text { code }\end{array}$ & $\begin{array}{c}\text { Depth } \\
(\mathrm{mbsf})\end{array}$ & $\delta^{18} \mathrm{O}$ & $\delta^{13} \mathrm{C}$ \\
\hline
\end{tabular}

Hole 677A (Cont.)

\begin{tabular}{|c|c|c|c|}
\hline A $87 / 1258$ & 6.10 & -2.48 & $\mathrm{~b}_{1.22}$ \\
\hline A $88 / 831$ & 6.10 & -2.18 & 1.16 \\
\hline A $87 / 1260$ & 6.59 & -1.14 & $b_{1.01}$ \\
\hline A $88 / 871$ & 6.59 & -1.23 & 0.82 \\
\hline A $87 / 1261$ & 6.68 & -1.12 & $\mathrm{~b}_{1.21}$ \\
\hline A $88 / 861$ & 6.68 & -0.84 & 1.05 \\
\hline A $87 / 1262$ & 6.80 & -1.09 & $b_{2.16}$ \\
\hline A $88 / 862$ & 6.80 & -0.86 & 1.23 \\
\hline A $87 / 1263$ & 6.92 & -1.62 & $\mathrm{~b}_{1.46}$ \\
\hline A $88 / 863$ & 6.92 & -1.81 & 0.97 \\
\hline A $87 / 1277$ & 7.18 & -2.34 & $\mathrm{~b}_{1.84}$ \\
\hline A $88 / 864$ & 7.18 & -1.64 & 1.60 \\
\hline A $87 / 1271$ & 7.29 & -1.81 & 1.75 \\
\hline A $88 / 865$ & 7.29 & -1.71 & 1.49 \\
\hline A $87 / 1272$ & 7.49 & -1.50 & 1.73 \\
\hline A $88 / 866$ & 7.49 & -1.47 & 1.55 \\
\hline A $87 / 1331$ & 7.59 & -1.61 & 1.65 \\
\hline A $88 / 867$ & 7.59 & -1.59 & 1.43 \\
\hline A $87 / 1278$ & 7.70 & -2.01 & $\mathrm{~b}_{1.50}$ \\
\hline A $87 / 1328$ & 7.70 & -1.60 & 1.90 \\
\hline A $87 / 1279$ & 7.80 & -2.08 & 1.31 \\
\hline A $87 / 1329$ & 7.80 & -0.14 & $b_{1.12}$ \\
\hline A $87 / 1330$ & 7.90 & -1.38 & 1.42 \\
\hline A $87 / 1315$ & 8.01 & -1.19 & 1.23 \\
\hline A $87 / 1313$ & 8.11 & -1.15 & 1.26 \\
\hline A $87 / 1312$ & 8.20 & -1.16 & 1.04 \\
\hline A $87 / 1311$ & 8.30 & -1.04 & 1.18 \\
\hline A $87 / 1332$ & 8.43 & -0.90 & 1.59 \\
\hline A $87 / 1335$ & 8.90 & -1.71 & 1.78 \\
\hline A $87 / 1336$ & 8.99 & -1.62 & 1.28 \\
\hline A $87 / 1316$ & 9.09 & -1.23 & 1.30 \\
\hline A $87 / 1317$ & 9.20 & -0.69 & 1.04 \\
\hline A $87 / 1318$ & 9.29 & -0.94 & 1.05 \\
\hline A $87 / 1319$ & 9.39 & -0.81 & 1.04 \\
\hline A $87 / 1320$ & 9.49 & -0 & 1.04 \\
\hline A $87 / 1339$ & 9.59 & -0.81 & 1.36 \\
\hline A $87 / 1338$ & 9.69 & -0.91 & 1.28 \\
\hline A $87 / 1337$ & 9.78 & -0.99 & 1.27 \\
\hline A $87 / 1342$ & 9.92 & -0.83 & 0.87 \\
\hline A $87 / 1343$ & 10.00 & -0.99 & 1.14 \\
\hline A $87 / 1344$ & 10.10 & -0.67 & 1.30 \\
\hline A $87 / 1345$ & 10.19 & -1.03 & 1.09 \\
\hline A $87 / 1346$ & 10.29 & -1 & 1.37 \\
\hline A $87 / 1347$ & 10.39 & -1.05 & 1.29 \\
\hline A $87 / 1348$ & 10.49 & -1.10 & 2.03 \\
\hline A $87 / 1350$ & 10.70 & -1.53 & 1.36 \\
\hline A $87 / 1351$ & 10.77 & -2.18 & 1.16 \\
\hline A $87 / 1352$ & 10.89 & -1.96 & 1.44 \\
\hline A $87 / 1353$ & 10.99 & -1.37 & 1.06 \\
\hline A $87 / 1354$ & 11.11 & -1.39 & 1.28 \\
\hline A $87 / 1355$ & 11.17 & -1.41 & 1.35 \\
\hline A $87 / 1356$ & 11.29 & -1.62 & 1.26 \\
\hline A $87 / 1357$ & 11.40 & -1.71 & 1.57 \\
\hline A $87 / 1358$ & 11.49 & -1.67 & 1.41 \\
\hline A $87 / 1359$ & 11.60 & -1.61 & 2.47 \\
\hline A $87 / 1891$ & 11.60 & -1.74 & 1.56 \\
\hline A $87 / 1360$ & 11.68 & -1.72 & 1.57 \\
\hline A $87 / 1361$ & 11.79 & -1.84 & 1.37 \\
\hline A $87 / 1362$ & 11.89 & -1.96 & 1.35 \\
\hline A $87 / 1363$ & 11.99 & -2.07 & 1.19 \\
\hline A $87 / 1364$ & 12.09 & -1.82 & 1.21 \\
\hline A $87 / 1365$ & 12.20 & -2.14 & 1.59 \\
\hline A $87 / 1366$ & 12.29 & -2.54 & 1.18 \\
\hline A $87 / 1367$ & 12.39 & -2.18 & 0.96 \\
\hline A $87 / 1368$ & 12.49 & -1.13 & 0.95 \\
\hline A $87 / 1369$ & 12.59 & -1.55 & $\mathrm{~b}_{3.43}$ \\
\hline A $87 / 1892$ & 12.59 & -0.92 & 1.27 \\
\hline A $87 / 1370$ & 12.69 & -0.37 & 0.98 \\
\hline A $87 / 1371$ & 12.79 & -0.55 & 0.92 \\
\hline A $87 / 1372$ & 12.92 & -0.57 & 0.90 \\
\hline A $87 / 1373$ & 13.01 & -0.90 & 0.96 \\
\hline A $87 / 1374$ & 13.10 & -1.21 & 0.93 \\
\hline A $87 / 1375$ & 13.18 & -0.77 & 1.32 \\
\hline A $87 / 1376$ & 13.30 & -1.20 & 1.05 \\
\hline A $87 / 1377$ & 13.40 & -0.82 & 1.50 \\
\hline A $87 / 1391$ & 13.52 & -0.97 & 1.17 \\
\hline
\end{tabular}

Table 2 (continued).

\begin{tabular}{|c|c|c|c|}
\hline $\begin{array}{l}\text { Sample } \\
\text { code }\end{array}$ & $\begin{array}{l}\text { Depth }^{a} \\
\text { (mbsf) }\end{array}$ & $\delta^{18} \mathrm{O}$ & $\delta^{13} \mathrm{C}$ \\
\hline \multicolumn{4}{|c|}{ Hole 677A (Cont.) } \\
\hline A $87 / 1380$ & 13.70 & -1.35 & 1.18 \\
\hline A $87 / 1381$ & 13.80 & -1.30 & 1.57 \\
\hline A $87 / 1382$ & 13.89 & -1.15 & 1.50 \\
\hline A $87 / 1383$ & 14.00 & -1.11 & 1.56 \\
\hline A $87 / 1384$ & 14.10 & -1.14 & 1.56 \\
\hline A $87 / 1385$ & 14.20 & -1.30 & 2.15 \\
\hline A $87 / 1386$ & 14.30 & -1.83 & 1.57 \\
\hline A $87 / 1387$ & 14.42 & -1.33 & 1.93 \\
\hline A $87 / 1388$ & 14.50 & -1.67 & 1.76 \\
\hline A $87 / 1389$ & 14.60 & -1.80 & 1.77 \\
\hline A $87 / 1390$ & 14.68 & -1.84 & 1.78 \\
\hline A $87 / 1392$ & 14.80 & -2.22 & 1.97 \\
\hline A $87 / 1393$ & 14.89 & -2.48 & 1.90 \\
\hline A $87 / 1394$ & 14.99 & -2.50 & 2.28 \\
\hline A $87 / 1395$ & 15.09 & -2.66 & 2.16 \\
\hline A $87 / 1396$ & 15.20 & -2.38 & 1.61 \\
\hline A $87 / 1397$ & 15.29 & -2.34 & 1.68 \\
\hline A $87 / 1398$ & 15.39 & -2.03 & 1.60 \\
\hline A $87 / 1432$ & 15.49 & -1.68 & 1.21 \\
\hline A $87 / 1400$ & 15.59 & -1.59 & 1.30 \\
\hline A $87 / 1434$ & 15.59 & -1.45 & 0.81 \\
\hline A $87 / 1401$ & 15.68 & -0.51 & 0.92 \\
\hline A $87 / 1402$ & 15.78 & -0.64 & 0.91 \\
\hline A $87 / 1403$ & 15.80 & -0.46 & 1.20 \\
\hline A $87 / 1404$ & 15.90 & -0.63 & 1.22 \\
\hline A $87 / 1405$ & 16.00 & -0.91 & 1.04 \\
\hline A $87 / 1406$ & 16.10 & -0.75 & 1.31 \\
\hline A $87 / 1436$ & 16.20 & -0.87 & 1.18 \\
\hline A $87 / 1438$ & 16.30 & -0.67 & 1.26 \\
\hline A $87 / 1409$ & 16.37 & -1.13 & 1.35 \\
\hline A $87 / 1440$ & 16.37 & -0.83 & 1.05 \\
\hline A $87 / 1410$ & 16.50 & -1.10 & 0.92 \\
\hline A $87 / 1411$ & 16.60 & -1.11 & 1.05 \\
\hline A $87 / 1412$ & 16.68 & -1.08 & 1.11 \\
\hline A $87 / 1413$ & 16.80 & -1.44 & 1.10 \\
\hline A $87 / 1414$ & 16.86 & -1.35 & 1.14 \\
\hline A $87 / 1415$ & 16.90 & -1.40 & 1.38 \\
\hline A $87 / 1416$ & 17.00 & -1.23 & 1.57 \\
\hline A $87 / 1441$ & 17.10 & -1.48 & 1.13 \\
\hline A $87 / 1418$ & 17.20 & -1.31 & 1.64 \\
\hline A $87 / 1419$ & 17.30 & -1.00 & 1.49 \\
\hline A $87 / 1421$ & 17.40 & -1.14 & 1.92 \\
\hline A $87 / 1422$ & 17.50 & -1.88 & 1.74 \\
\hline A $87 / 1423$ & 17.60 & -1.55 & 1.94 \\
\hline A $87 / 1424$ & 17.70 & -2.04 & 1.85 \\
\hline A $87 / 1425$ & 17.80 & -1.78 & 2.00 \\
\hline A $87 / 1426$ & 17.87 & -1.62 & 2.20 \\
\hline A $87 / 1427$ & 18.00 & -1.33 & 2.15 \\
\hline A $87 / 1428$ & 18.10 & -1.66 & 1.96 \\
\hline A $87 / 1429$ & 18.18 & -1.30 & 2.15 \\
\hline A $87 / 1430$ & 18.30 & -1.69 & 1.92 \\
\hline A $87 / 1442$ & 18.40 & -1.62 & 1.60 \\
\hline A $87 / 1443$ & 18.50 & -1.50 & 1.81 \\
\hline A $87 / 1444$ & 18.60 & -1.53 & 1.69 \\
\hline A $87 / 1445$ & 18.70 & -1.30 & 1.70 \\
\hline A $87 / 1446$ & 18.80 & -1.17 & 1.91 \\
\hline A $87 / 1447$ & 18.90 & -1.32 & 1.64 \\
\hline A $87 / 1448$ & 19.00 & -1.34 & 1.56 \\
\hline A $87 / 1449$ & 19.10 & -1.40 & 1.62 \\
\hline A $87 / 1450$ & 19.20 & -1.43 & 1.48 \\
\hline A $87 / 1451$ & 19.29 & -1.69 & 1.41 \\
\hline A $87 / 1452$ & 19.37 & -1.63 & 1.33 \\
\hline A $87 / 1453$ & 19.48 & -1.52 & 1.29 \\
\hline A $87 / 1454$ & 19.59 & -1.52 & 1.03 \\
\hline A $87 / 1455$ & 19.67 & -1.29 & 1.37 \\
\hline A $87 / 1456$ & 19.79 & -1.10 & 1.20 \\
\hline A $87 / 1457$ & 19.90 & -1.42 & 0.96 \\
\hline A $87 / 1458$ & 20.00 & -1.31 & 0.79 \\
\hline A $87 / 1459$ & 20.10 & -1.36 & 1.15 \\
\hline A $87 / 1460$ & 20.20 & -1.67 & 1.23 \\
\hline A $87 / 1461$ & 20.30 & -1.55 & 1.10 \\
\hline A $87 / 1462$ & 20.40 & -1.66 & 1.27 \\
\hline A $87 / 1463$ & 20.50 & -2.15 & 1.23 \\
\hline A $87 / 1464$ & 20.60 & -1.96 & 1.28 \\
\hline A $87 / 1465$ & 20.70 & -2.23 & 1.23 \\
\hline A $87 / 1466$ & 20.80 & -1.92 & 1.43 \\
\hline
\end{tabular}


Table 2 (continued).

\begin{tabular}{|c|c|c|c|}
\hline $\begin{array}{l}\text { Sample } \\
\text { code }\end{array}$ & $\begin{array}{l}\text { Depth }^{a} \\
\text { (mbsf) }\end{array}$ & $\delta^{18} \mathrm{O}$ & $\delta^{13} \mathrm{C}$ \\
\hline \multicolumn{4}{|c|}{ Hole 677A (Cont.) } \\
\hline A $87 / 1467$ & 20.87 & -2.16 & 1.58 \\
\hline A $87 / 1468$ & 21.00 & -2.20 & 1.34 \\
\hline A $87 / 1469$ & 21.09 & -1.66 & 0.93 \\
\hline A $87 / 1470$ & 21.20 & -1.55 & 1.25 \\
\hline A $87 / 1471$ & 21.30 & -1.51 & 1.14 \\
\hline A $87 / 1472$ & 21.70 & -1.97 & 1.34 \\
\hline A $87 / 1473$ & 21.80 & -1.93 & 1.36 \\
\hline A $89 / 92$ & 21.80 & -1.58 & 1.46 \\
\hline A $87 / 1474$ & 21.90 & -1.68 & 1.40 \\
\hline A $89 / 93$ & 21.90 & -1.68 & 1.43 \\
\hline A $87 / 1475$ & 22.00 & -1.78 & 1.54 \\
\hline A $89 / 94$ & 22.00 & -1.58 & 1.44 \\
\hline A $87 / 1476$ & 22.10 & -1.85 & 1.48 \\
\hline A $89 / 95$ & 22.10 & -1.72 & 1.37 \\
\hline A $87 / 1477$ & 22.20 & -1.99 & 1.47 \\
\hline A $89 / 96$ & 22.20 & -2.23 & 1.44 \\
\hline A $87 / 1478$ & 22.30 & -2.08 & 1.24 \\
\hline A $89 / 97$ & 22.30 & -2.02 & 1.29 \\
\hline A $87 / 1479$ & 22.37 & -2.39 & 0.94 \\
\hline A $89 / 98$ & 22.37 & -1.78 & 0.92 \\
\hline A $87 / 1480$ & 22.50 & -1.32 & 0.88 \\
\hline A $89 / 99$ & 22.50 & -0.98 & 0.97 \\
\hline A $87 / 1481$ & 22.60 & -0.83 & 1.00 \\
\hline A $89 / 100$ & 22.60 & -0.92 & 0.91 \\
\hline A $87 / 1482$ & 22.67 & -0.39 & 0.89 \\
\hline A $89 / 101$ & 22.67 & -0.43 & 0.81 \\
\hline A $87 / 1483$ & 22.80 & -0.19 & 0.86 \\
\hline A $89 / 102$ & 22.80 & -0.52 & 0.75 \\
\hline A $87 / 1484$ & 22.90 & -0.29 & 0.85 \\
\hline A $89 / 103$ & 22.90 & -0.31 & 0.95 \\
\hline A $87 / 1485$ & 23.00 & -0.57 & 0.81 \\
\hline A $89 / 104$ & 23.00 & -0.71 & 0.83 \\
\hline A $87 / 1486$ & 23.10 & -0.59 & 1.12 \\
\hline A $89 / 105$ & 23.10 & -0.48 & 0.92 \\
\hline A $87 / 1487$ & 23.20 & -0.30 & 1.00 \\
\hline A $89 / 106$ & 23.20 & -0.71 & 1.12 \\
\hline A $87 / 1488$ & 23.30 & -0.46 & 1.00 \\
\hline A $89 / 107$ & 23.30 & -0.65 & 1.06 \\
\hline A $87 / 1489$ & 23.40 & -0.60 & 0.90 \\
\hline A $89 / 108$ & 23.40 & -0.52 & 0.88 \\
\hline A $87 / 1490$ & 23.50 & -1.01 & 0.95 \\
\hline A $89 / 109$ & 23.50 & -0.95 & 1.03 \\
\hline A $87 / 1491$ & 23.60 & -0.75 & 0.82 \\
\hline A $89 / 110$ & 23.60 & -0.80 & 0.90 \\
\hline A $87 / 1492$ & 23.70 & -0.55 & 0.77 \\
\hline A $89 / 111$ & 23.70 & -0.65 & 0.60 \\
\hline A $87 / 1493$ & 23.80 & -0.71 & 0.76 \\
\hline A $89 / 112$ & 23.80 & -0.92 & 0.90 \\
\hline A $87 / 1494$ & 23.87 & -0.65 & 0.96 \\
\hline A $89 / 113$ & 23.87 & -0.77 & 0.88 \\
\hline A $87 / 1495$ & 24.00 & -0.56 & 1.00 \\
\hline A $89 / 114$ & 24.00 & -0.66 & 0.80 \\
\hline A $87 / 1496$ & 24.09 & -0.34 & 0.93 \\
\hline A $89 / 115$ & 24.09 & -0.97 & 0.86 \\
\hline A $87 / 1497$ & 24.18 & -0.77 & 0.96 \\
\hline A $89 / 116$ & 24.18 & -1.23 & 0.87 \\
\hline A $87 / 1501$ & 24.29 & -1.15 & 1.05 \\
\hline A $89 / 117$ & 24.29 & -1.27 & 1.10 \\
\hline A $87 / 1502$ & 24.39 & -0.98 & 1.03 \\
\hline A $89 / 118$ & 24.39 & -1.04 & 1.12 \\
\hline A $87 / 1503$ & 24.49 & -1.29 & 1.09 \\
\hline A $89 / 119$ & 24.49 & -1.39 & 1.33 \\
\hline A $87 / 1504$ & 24.59 & -1.42 & 0.95 \\
\hline A $89 / 120$ & 24.59 & -1.50 & 1.27 \\
\hline A $87 / 1505$ & 24.70 & -1.22 & 1.16 \\
\hline A $89 / 121$ & 24.70 & -1.40 & 1.27 \\
\hline A $87 / 1506$ & 24.80 & -1.46 & 1.11 \\
\hline A $89 / 122$ & 24.80 & -1.37 & 1.14 \\
\hline A $87 / 1507$ & 24.90 & -1.50 & 1.18 \\
\hline A $89 / 123$ & 24.90 & -1.80 & 1.37 \\
\hline A $87 / 1508$ & 25.00 & -1.87 & 1.42 \\
\hline A $89 / 124$ & 25.00 & -1.74 & 1.58 \\
\hline A $87 / 1509$ & 25.09 & -1.88 & 1.57 \\
\hline A $89 / 125$ & 25.09 & -1.91 & 1.58 \\
\hline A $87 / 1510$ & 25.19 & -2.09 & 1.41 \\
\hline A $89 / 126$ & 25.19 & -1.91 & 1.71 \\
\hline
\end{tabular}

Table 2 (continued).

\begin{tabular}{cccc}
\hline $\begin{array}{c}\text { Sample } \\
\text { code }\end{array}$ & $\begin{array}{c}\text { Depth } \\
\text { (mbsf) }\end{array}$ & $\delta^{18} \mathrm{O}$ & $\delta^{13} \mathrm{C}$ \\
\hline
\end{tabular}

Hole 677A (Cont.)

$$
\begin{array}{llll}
\text { A } 87 / 1530 & 25.29 & -1.74 & 1.45
\end{array}
$$$$
\begin{array}{llll}
\text { A } 8 \text { S. } 1.7 & 25.29 & -1.83 & 1.56
\end{array}
$$$$
\begin{array}{llll}
\text { A } 87 / 1631 & 25.31 & -0.92 & 1.20
\end{array}
$$$$
\begin{array}{llll}
\text { A } 87 / 1632 & 25.39 & -1.11 & 1.26
\end{array}
$$$$
\begin{array}{llll}
\text { A } 87 / 1633 & 25.49 & -1.28 & 1.46
\end{array}
$$$$
\begin{array}{llll}
\text { A } 87 / 1634 & 25.59 & -1.42 & 1.33
\end{array}
$$$$
\begin{array}{llll}
\text { A } 87 / 1635 & 25.66 & -1.36 & 1.22
\end{array}
$$$$
\begin{array}{llll}
\text { A } 87 / 1636 & 26.02 & -0.98 & 0.82
\end{array}
$$$$
\begin{array}{llll}
\text { A } 87 / 1637 & 26.10 & -1.06 & 0.90
\end{array}
$$$$
\begin{array}{llll}
\text { A } 87 / 1638 & 26.20 & -0.94 & 0.60
\end{array}
$$$$
\begin{array}{llll}
\text { A } 87 / 1639 & 26.30 & -0.96 & 1.06
\end{array}
$$$$
\begin{array}{llll}
\text { A } 87 / 1640 & 26.37 & -0.80 & 1.20
\end{array}
$$$$
\begin{array}{llll}
\text { A } 87 / 1641 & 26.50 & -1.10 & 1.38
\end{array}
$$$$
\begin{array}{llll}
\text { A } 87 / 1642 & 26.60 & -1.14 & 1.33
\end{array}
$$$$
\begin{array}{llll}
\text { A } 87 / 1643 & 26.71 & -1.07 & 1.40
\end{array}
$$$$
\begin{array}{llll}
\text { A } 87 / 1644 & 26.80 & -1.14 & 1.06
\end{array}
$$$$
\begin{array}{llll}
\text { A } 87 / 1645 & 26.90 & -1.39 & 1.24
\end{array}
$$$$
\begin{array}{llll}
\text { A } 87 / 1646 & 27.00 & -1.31 & 1.55
\end{array}
$$$$
\begin{array}{llll}
\text { A } 87 / 1647 & 27.10 & -1.74 & 1.47
\end{array}
$$$$
\begin{array}{llll}
\text { A } 87 / 1648 & 27.19 & -1.57 & 1.68
\end{array}
$$$$
\begin{array}{llll}
\text { A } 87 / 1649 & 27.30 & -1.77 & 1.57
\end{array}
$$$$
\begin{array}{llll}
\text { A } 87 / 1650 & 27.41 & -1.72 & 1.62
\end{array}
$$$$
\begin{array}{llll}
\text { A } 87 / 1671 & 27.49 & -1.71 & 1.46
\end{array}
$$$$
\begin{array}{llll}
\text { A } 87 / 1672 & 27.56 & -1.74 & 1.67
\end{array}
$$$$
\begin{array}{llll}
\text { A } 87 / 1673 & 27.68 & -2.11 & 1.45
\end{array}
$$$$
\begin{array}{llll}
\text { A } 87 / 1674 & 27.80 & -1.63 & 1.35
\end{array}
$$$$
\begin{array}{llll}
\text { A } 87 / 1675 & 27.90 & -1.47 & 1.19
\end{array}
$$$$
\begin{array}{llll}
\text { A } 87 / 1676 & 27.99 & -1.13 & 1.00
\end{array}
$$$$
\begin{array}{llll}
\text { A } 87 / 1677 & 28.10 & -0.66 & 1.12
\end{array}
$$$$
\begin{array}{llll}
\text { A } 87 / 1678 & 28.20 & -0.62 & 1.04
\end{array}
$$$$
\begin{array}{llll}
\text { A } 87 / 1679 & 28.30 & -0.67 & 1.05
\end{array}
$$$$
\begin{array}{llll}
\text { A } 87 / 1680 & 28.40 & -0.73 & 1.10
\end{array}
$$$$
\begin{array}{llll}
\text { A } 87 / 1681 & 28.50 & -0.49 & 1.38
\end{array}
$$$$
\begin{array}{llll}
\text { A } 87 / 1682 & 28.60 & -0.88 & 1.52
\end{array}
$$$$
\begin{array}{llll}
\text { A } 87 / 1683 & 28.70 & -1.07 & 1.31
\end{array}
$$$$
\begin{array}{llll}
\text { A } 87 / 1684 & 28.77 & -1.18 & 1.23
\end{array}
$$$$
\begin{array}{llll}
\text { A } 87 / 1685 & 28.87 & -1.08 & 1.55
\end{array}
$$$$
\begin{array}{llll}
\text { A } 87 / 1686 & 28.99 & -1.09 & 1.57
\end{array}
$$$$
\begin{array}{llll}
\text { A } 87 / 1687 & 29.09 & -1.32 & 1.59
\end{array}
$$$$
\begin{array}{llll}
\text { A } 87 / 1688 & 29.18 & -1.31 & 1.42
\end{array}
$$$$
\begin{array}{llll}
\text { A } 87 / 1736 & 29.29 & -1.27 & 1.28
\end{array}
$$$$
\begin{array}{llll}
\text { A } 87 / 1689 & 29.40 & -1.67 & 1.19
\end{array}
$$$$
\begin{array}{llll}
\text { A } 87 / 1690 & 29.50 & -1.38 & 1.47
\end{array}
$$$$
\begin{array}{llll}
\text { A } 87 / 1691 & 29.60 & -1.61 & 1.16
\end{array}
$$$$
\begin{array}{llll}
\text { A } 87 / 1692 & 29.70 & -1.88 & 0.93
\end{array}
$$$$
\begin{array}{llll}
\text { A } 87 / 1693 & 29.80 & -1.74 & 0.94
\end{array}
$$$$
\begin{array}{llll}
\text { A } 87 / 1694 & 29.90 & -1.39 & 1.21
\end{array}
$$$$
\begin{array}{llll}
\text { A } 87 / 1695 & 30.00 & -1.71 & 1.33
\end{array}
$$$$
\begin{array}{llll}
\text { A } 87 / 1696 & 30.10 & -1.62 & 1.39
\end{array}
$$$$
\begin{array}{llll}
\text { A } 87 / 1697 & 30.20 & -1.75 & 1.51
\end{array}
$$$$
\begin{array}{llll}
\text { A } 87 / 1698 & 30.30 & -1.83 & 1.31
\end{array}
$$$$
\begin{array}{llll}
\text { A } 87 / 1699 & 30.40 & -1.60 & 1.21
\end{array}
$$$$
\begin{array}{llll}
\text { A } 88 / 845 & 30.40 & -1.75 & 1.12
\end{array}
$$$$
\begin{array}{llll}
\text { A } 87 / 1700 & 30.50 & -1.39 & 1.25
\end{array}
$$$$
\begin{array}{llll}
\text { A } 88 / 780 & 30.50 & -1.44 & 1.01
\end{array}
$$$$
\begin{array}{llll}
\text { A } 87 / 1713 & 30.59 & -1.61 & 1.21
\end{array}
$$$$
\begin{array}{llll}
\text { A } 87 / 1702 & 30.68 & -1.65 & 1.31
\end{array}
$$$$
\begin{array}{llll}
\text { A } 87 / 1703 & 30.80 & -1.65 & 1.23 \\
\text { A } 87 / 1704 & 30.90 & -1.65 & 1.27
\end{array}
$$$$
\begin{array}{llll}
\text { A } 87 / 1705 & 31.00 & -1.92 & 1.10
\end{array}
$$$$
\begin{array}{llll}
\text { A } 87 / 1706 & 31.10 & -1.70 & 0.78
\end{array}
$$$$
\begin{array}{llll}
\text { A } 87 / 1707 & 31.20 & -0.64 & 0.90
\end{array}
$$$$
\begin{array}{llll}
\text { A } 89 / 157 & 31.20 & -0.89 & 0.85
\end{array}
$$$$
\begin{array}{llll}
\text { A } 87 / 1708 & 31.30 & -0.35 & 0.74
\end{array}
$$$$
\begin{array}{llll}
\text { A } 89 / 128 & 31.30 & -0.83 & 1.00
\end{array}
$$$$
\begin{array}{llll}
\text { A } 87 / 1711 & 31.40 & -0.36 & 0.36
\end{array}
$$$$
\begin{array}{llll}
\text { A } 89 / 130 & 31.40 & -0.35 & 0.58
\end{array}
$$$$
\begin{array}{llll}
\text { A } 87 / 1709 & 31.50 & -0.46 & 0.66
\end{array}
$$$$
\begin{array}{llll}
\text { A } 89 / 131 & 31.50 & -0.27 & 0.59 \\
\text { A } 87 / 1710 & 31.60 & -0.28 & 0.89
\end{array}
$$$$
\begin{array}{llll}
\text { A } 87 / 1710 & 31.60 & -0.28 & 0.89
\end{array}
$$$$
\begin{array}{llll}
\text { A } 89 / 132 & 31.60 & -0.40 & 0.71 \\
\text { A } 87 / 1712 & 31.70 & -0.74 & 0.83
\end{array}
$$$$
\begin{array}{llll}
\text { A } 87 / 1712 & 31.70 & -0.74 & 0.83 \\
\text { A } 89 / 133 & 31.70 & -0.81 & 0.82
\end{array}
$$$$
\begin{array}{llll}
\text { A } 87 / 1714 & 31.80 & -0.70 & 0.87
\end{array}
$$$$
\begin{array}{llll}
\text { A } 89 / 134 & 31.80 & -0.95 & 0.87
\end{array}
$$$$
\begin{array}{llll}
\text { A } 87 / 1715 & 31.92 & -0.10 & 1.22
\end{array}
$$

Table 2 (continued).

\begin{tabular}{|c|c|c|c|}
\hline $\begin{array}{l}\text { Sample } \\
\text { code }\end{array}$ & $\begin{array}{l}\text { Depth }^{a} \\
\text { (mbsf) }\end{array}$ & $\delta^{18} \mathrm{O}$ & $\delta^{13} \mathrm{C}$ \\
\hline \multicolumn{4}{|c|}{ Hole 677A (Cont.) } \\
\hline A $89 / 135$ & 31.92 & -0.70 & 0.78 \\
\hline A $87 / 1716$ & 32.00 & -0.64 & 0.81 \\
\hline A $89 / 136$ & 32.00 & -0.74 & 0.84 \\
\hline A $87 / 1717$ & 32.10 & -0.82 & 0.57 \\
\hline A $89 / 137$ & 32.10 & -0.69 & 0.84 \\
\hline A $87 / 1718$ & 32.18 & -0.69 & 0.75 \\
\hline A $89 / 138$ & 32.18 & -0.78 & 0.58 \\
\hline A $87 / 1719$ & 32.30 & -0.71 & 0.71 \\
\hline A $89 / 139$ & 32.30 & -0.74 & 0.79 \\
\hline A $87 / 1720$ & 32.40 & -0.89 & 0.85 \\
\hline A $89 / 140$ & 32.40 & -0.96 & 0.93 \\
\hline A $87 / 1721$ & 32.50 & -1.22 & 0.75 \\
\hline A $89 / 141$ & 32.50 & -1.18 & 0.91 \\
\hline A $87 / 1722$ & 32.60 & -0.96 & 0.94 \\
\hline A $89 / 142$ & 32.60 & -1.01 & 0.82 \\
\hline A $87 / 1723$ & 32.70 & -1.02 & 1.06 \\
\hline A $89 / 143$ & 32.70 & -1.32 & 1.03 \\
\hline A $87 / 1724$ & 32.80 & -1.35 & 0.97 \\
\hline A $89 / 144$ & 32.80 & -1.45 & 0.90 \\
\hline A $87 / 1725$ & 32.90 & -1.09 & 0.70 \\
\hline A $89 / 145$ & 32.90 & -1.34 & 0.69 \\
\hline A $87 / 1726$ & 33.00 & -1.05 & 0.69 \\
\hline A $89 / 146$ & 33.00 & -1.26 & 0.69 \\
\hline A $87 / 1727$ & 33.10 & -0.81 & 0.41 \\
\hline A $87 / 1728$ & 33.30 & -0.92 & 0.64 \\
\hline A $89 / 148$ & 33.30 & -0.95 & 0.83 \\
\hline A $87 / 1729$ & 33.40 & -0.77 & 0.98 \\
\hline A $89 / 149$ & 33.40 & -1.20 & 0.79 \\
\hline A $87 / 1861$ & 33.50 & -0.84 & 1.10 \\
\hline A $89 / 150$ & 33.50 & -1.00 & 1.04 \\
\hline A $87 / 1730$ & 33.60 & -1.11 & 0.90 \\
\hline A $89 / 151$ & 33.60 & -1.14 & 0.88 \\
\hline A $89 / 152$ & 33.68 & -1.43 & 1.25 \\
\hline A $87 / 1732$ & 33.80 & -1.35 & 1.59 \\
\hline A $89 / 153$ & 33.80 & -1.48 & 1.31 \\
\hline A $87 / 1733$ & 33.90 & -1.21 & 1.60 \\
\hline A $89 / 154$ & 33.90 & -1.24 & 1.26 \\
\hline A $87 / 1734$ & 34.00 & -1.16 & 1.57 \\
\hline A $89 / 155$ & 34.00 & -1.44 & 1.46 \\
\hline A $87 / 1735$ & 34.10 & -1.25 & 1.60 \\
\hline A $89 / 156$ & 34.10 & -1.40 & 1.35 \\
\hline A $87 / 1473$ & 21.80 & -1.93 & 1.36 \\
\hline A $87 / 1474$ & 21.90 & -1.68 & 1.40 \\
\hline A $87 / 1475$ & 22.00 & -1.78 & 1.54 \\
\hline A $87 / 1476$ & 22.10 & -1.85 & 1.48 \\
\hline A $87 / 1477$ & 22.20 & -1.99 & 1.47 \\
\hline A $87 / 1478$ & 22.30 & -2.08 & 1.24 \\
\hline A $87 / 1479$ & 22.37 & -2.39 & 0.94 \\
\hline A $87 / 1480$ & 22.50 & -1.32 & 0.88 \\
\hline A $87 / 1481$ & 22.60 & -0.83 & 1.00 \\
\hline A $87 / 1482$ & 22.67 & -0.39 & 0.89 \\
\hline A $87 / 1483$ & 22.80 & -0.19 & 0.86 \\
\hline A $87 / 1484$ & 22.90 & -0.29 & 0.85 \\
\hline A $87 / 1485$ & 23.00 & -0.57 & 0.81 \\
\hline A $87 / 1486$ & 23.10 & -0.59 & 1.12 \\
\hline A $87 / 1487$ & 23.20 & -0.30 & 1.00 \\
\hline A $87 / 1488$ & 23.30 & -0.46 & 1.00 \\
\hline A $87 / 1489$ & 23.40 & -0.60 & 0.90 \\
\hline A $87 / 1490$ & 23.50 & -1.01 & 0.95 \\
\hline A $87 / 1491$ & 23.60 & -0.75 & 0.82 \\
\hline A $87 / 1492$ & 23.70 & -0.55 & 0.77 \\
\hline A $87 / 1493$ & 23.80 & -0.71 & 0.76 \\
\hline A $87 / 1494$ & 23.87 & -0.65 & 0.96 \\
\hline A $87 / 1495$ & 24.00 & -0.56 & 1.00 \\
\hline A $87 / 1496$ & 24.09 & -0.34 & 0.93 \\
\hline A $87 / 1497$ & 24.18 & -0.77 & 0.96 \\
\hline A $87 / 1501$ & 24.29 & -1.15 & 1.05 \\
\hline A $87 / 1502$ & 24.39 & -0.98 & 1.03 \\
\hline A $87 / 1503$ & 24.49 & -1.29 & 1.09 \\
\hline A $87 / 1504$ & 24.59 & -1.42 & 0.95 \\
\hline A $87 / 1505$ & 24.70 & -1.22 & 1.16 \\
\hline A $87 / 1506$ & 24.80 & -1.46 & 1.11 \\
\hline A $87 / 1507$ & 24.90 & -1.50 & 1.18 \\
\hline A $87 / 1508$ & 25.00 & -1.87 & 1.42 \\
\hline A $87 / 1509$ & 25.09 & -1.88 & 1.57 \\
\hline A $87 / 1510$ & 25.19 & -2.09 & 1.41 \\
\hline
\end{tabular}


Table 2 (continued).

\begin{tabular}{|c|c|c|c|}
\hline $\begin{array}{l}\text { Sample } \\
\text { code }\end{array}$ & $\begin{array}{l}\text { Depth }^{a} \\
\text { (mbsf) }\end{array}$ & $\delta^{18} \mathrm{O}$ & $\delta^{13} \mathrm{C}$ \\
\hline \multicolumn{4}{|c|}{ Hole $677 \mathrm{~A}$ (Cont.) } \\
\hline A $87 / 1530$ & 25.29 & -1.74 & 1.45 \\
\hline A $87 / 1631$ & 25.31 & -0.92 & 1.20 \\
\hline A $87 / 1632$ & 25.39 & -1.11 & 1.26 \\
\hline A $87 / 1633$ & 25.49 & -1.28 & 1.46 \\
\hline A $87 / 1634$ & 25.59 & -1.42 & 1.33 \\
\hline A $87 / 1635$ & 25.66 & -1.36 & 1.22 \\
\hline A $87 / 1636$ & 26.02 & -0.98 & 0.82 \\
\hline A $87 / 1637$ & 26.10 & -1.06 & 0.90 \\
\hline A $87 / 1638$ & 26.20 & -0.94 & 0.60 \\
\hline A $87 / 1639$ & 26.30 & -0.96 & 1.06 \\
\hline A $87 / 1640$ & 26.37 & -0.80 & 1.20 \\
\hline A $87 / 1641$ & 26.50 & -1.10 & 1.38 \\
\hline A $87 / 1642$ & 26.60 & -1.14 & 1.33 \\
\hline A $87 / 1643$ & 26.71 & -1.07 & 1.40 \\
\hline A $87 / 1644$ & 26.80 & -1.14 & 1.06 \\
\hline A $87 / 1645$ & 26.90 & -1.39 & 1.24 \\
\hline A $87 / 1646$ & 27.00 & -1.31 & 1.55 \\
\hline A $87 / 1647$ & 27.10 & -1.74 & 1.47 \\
\hline A $87 / 1648$ & 27.19 & -1.57 & 1.68 \\
\hline A $87 / 1649$ & 27.30 & -1.77 & 1.57 \\
\hline A $87 / 1650$ & 27.41 & -1.72 & 1.62 \\
\hline A $87 / 1671$ & 27.49 & -1.71 & 1.46 \\
\hline A $87 / 1672$ & 27.56 & -1.74 & 1.67 \\
\hline A $87 / 1673$ & 27.68 & -2.11 & 1.45 \\
\hline A $87 / 1674$ & 27.80 & -1.63 & 1.35 \\
\hline A $87 / 1675$ & 27.90 & -1.47 & 1.19 \\
\hline A $87 / 1676$ & 27.99 & -1.13 & 1.00 \\
\hline A $87 / 1677$ & 28.10 & -0.66 & 1.12 \\
\hline A $87 / 1678$ & 28.20 & -0.62 & 1.04 \\
\hline A $87 / 1679$ & 28.30 & -0.67 & 1.05 \\
\hline A $87 / 1680$ & 28.40 & -0.73 & 1.10 \\
\hline A $87 / 1681$ & 28.50 & -0.49 & 1.38 \\
\hline A $87 / 1682$ & 28.60 & -0.88 & 1.52 \\
\hline A $87 / 1683$ & 28.70 & -1.07 & 1.31 \\
\hline A $87 / 1684$ & 28.77 & -1.18 & 1.23 \\
\hline A $87 / 1685$ & 28.87 & -1.08 & 1.55 \\
\hline A $87 / 1686$ & 28.99 & -1.09 & 1.57 \\
\hline A $87 / 1687$ & 29.09 & -1.32 & 1.59 \\
\hline A $87 / 1688$ & 29.18 & -1.31 & 1.42 \\
\hline A $87 / 1736$ & 29.29 & -1.27 & 1.28 \\
\hline A $87 / 1689$ & 29.40 & -1.67 & 1.19 \\
\hline A $87 / 1690$ & 29.50 & -1.38 & 1.47 \\
\hline A $87 / 1691$ & 29.60 & -1.61 & 1.16 \\
\hline A $87 / 1692$ & 29.70 & -1.88 & 0.93 \\
\hline A $87 / 1693$ & 29.80 & -1.74 & 0.94 \\
\hline A $87 / 1694$ & 29.90 & -1.39 & 1.21 \\
\hline A $87 / 1695$ & 30.00 & -1.71 & 1.33 \\
\hline A $87 / 1696$ & 30.10 & -1.62 & 1.39 \\
\hline A $87 / 1697$ & 30.20 & -1.75 & 1.51 \\
\hline A $87 / 1698$ & 30.30 & -1.83 & 1.31 \\
\hline A $87 / 1699$ & 30.40 & -1.60 & 1.21 \\
\hline A $88 / 845$ & 30.40 & -1.75 & 1.12 \\
\hline A $87 / 1700$ & 30.50 & -1.39 & 1.25 \\
\hline A $88 / 780$ & 30.50 & -1.44 & 1.01 \\
\hline A $87 / 1713$ & 30.59 & -1.61 & 1.21 \\
\hline A $87 / 1702$ & 30.68 & -1.65 & 1.31 \\
\hline A $87 / 1703$ & 30.80 & -1.65 & 1.23 \\
\hline A $87 / 1704$ & 30.90 & -1.65 & 1.27 \\
\hline A $87 / 1705$ & 31.00 & -1.92 & 1.10 \\
\hline A $87 / 1706$ & 31.10 & -1.70 & 0.78 \\
\hline A $87 / 1707$ & 31.20 & -0.64 & 0.90 \\
\hline A $87 / 1708$ & 31.30 & -0.35 & 0.74 \\
\hline A $87 / 1711$ & 31.40 & -0.36 & 0.36 \\
\hline A $87 / 1709$ & 31.50 & -0.46 & 0.66 \\
\hline A $87 / 1710$ & 31.60 & -0.28 & 0.89 \\
\hline A $87 / 1712$ & 31.70 & -0.74 & 0.83 \\
\hline A $87 / 1714$ & 31.80 & -0.70 & 0.87 \\
\hline A $87 / 1715$ & 31.92 & -0.10 & 1.22 \\
\hline A $87 / 1716$ & 32.00 & -0.64 & 0.81 \\
\hline A $87 / 1717$ & 32.10 & -0.82 & 0.57 \\
\hline A $87 / 1718$ & 32.18 & -0.69 & 0.75 \\
\hline A $87 / 1719$ & 32.30 & -0.71 & 0.71 \\
\hline A $87 / 1720$ & 32.40 & -0.89 & 0.85 \\
\hline A $87 / 1721$ & 32.50 & -1.22 & 0.75 \\
\hline A $87 / 1722$ & 32.60 & -0.96 & 0.94 \\
\hline A $87 / 1723$ & 32.70 & -1.02 & 1.06 \\
\hline
\end{tabular}

Table 2 (continued).

\begin{tabular}{|c|c|c|}
\hline $\begin{array}{l}\text { Sample } \\
\text { code }\end{array}$ & $\begin{array}{l}\text { Deptha } \\
\text { (mbsf) }\end{array}$ & $\delta^{18} \mathrm{O}$ \\
\hline
\end{tabular}

$\begin{array}{llll}\text { A } 87 / 1724 & 32.80 & -1.35 & 0.97\end{array}$

$\begin{array}{llll}\text { A } 87 / 1725 & 32.90 & -1.09 & 0.70\end{array}$

$\begin{array}{llll}\text { A } 87 / 1726 & 33.00 & -1.05 & 0.69\end{array}$

$\begin{array}{llll}\text { A } 87 / 1727 & 33.10 & -0.81 & 0.41\end{array}$

$\begin{array}{llll}\text { A } 87 / 1728 & 33.30 & -0.92 & 0.64\end{array}$

$\begin{array}{llll}\text { A } 87 / 1729 & 33.40 & -0.77 & 0.98\end{array}$

$\begin{array}{llll}\text { A } 87 / 1861 & 33.50 & -0.84 & 1.10\end{array}$

$\begin{array}{llll}\text { A } 87 / 1730 & 33.60 & -1.11 & 0.90\end{array}$

$\begin{array}{llll}\text { A } 87 / 1732 & 33.80 & -1.35 & 1.59\end{array}$

$\begin{array}{llll}\text { A } 87 / 1733 & 33.90 & -1.21 & 1.60\end{array}$

$\begin{array}{llll}\text { A } 87 / 1734 & 34.00 & -1.16 & 1.57\end{array}$

$\begin{array}{llll}\text { A } 87 / 1735 & 34.10 & -1.25 & 1.60\end{array}$

$\begin{array}{llll}\text { A } 87 / 1737 & 34.80 & -0.63 & 0.80\end{array}$

$\begin{array}{llll}\text { A } 87 / 1738 & 34.90 & -0.95 & 0.78\end{array}$

$\begin{array}{llll}\text { A } 87 / 1739 & 35.00 & -0.73 & 1.00\end{array}$

$\begin{array}{llll}\text { A } 87 / 1740 & 35.10 & -0.71 & 1.17\end{array}$

$\begin{array}{llll}\text { A } 87 / 1762 & 35.50 & -1.07 & 1.30\end{array}$

$\begin{array}{llll}\text { A } 87 / 1763 & 35.60 & -1.59 & 1.40\end{array}$

$\begin{array}{llll}\text { A } 87 / 1764 & 35.70 & -1.45 & 1.68\end{array}$

$\begin{array}{llll}\text { A } 87 / 1765 & 35.90 & -1.46 & 1.73\end{array}$

$\begin{array}{llll}\text { A } 87 / 1766 & 36.00 & -1.65 & 1.46\end{array}$

$\begin{array}{llll}\text { A } 87 / 1768 & 36.10 & -1.82 & 1.42\end{array}$

$\begin{array}{llll}\text { A } 87 / 1769 & 36.21 & -2.28 & 1.23\end{array}$

$\begin{array}{llll}\text { A } 87 / 1770 & 36.30 & -1.82 & 1.09\end{array}$

$\begin{array}{llll}\text { A } 87 / 1781 & 36.40 & -1.75 & 1.07\end{array}$

$\begin{array}{llll}\text { A } 87 / 1782 & 36.50 & -1.61 & 0.97\end{array}$

$\begin{array}{llll}\text { A } 87 / 1783 & 36.60 & -1.11 & 0.69\end{array}$

$\begin{array}{llll}\text { A } 87 / 1784 & 36.70 & -0.83 & 1.36\end{array}$

$\begin{array}{llll}\text { A } 87 / 1785 & 36.80 & -1.17 & 1.24\end{array}$

$\begin{array}{llll}\text { A } 87 / 1786 & 36.87 & -0.85 & 1.58\end{array}$

$\begin{array}{llll}\text { A } 87 / 1787 & 37.00 & -2.45 & b_{1} .55\end{array}$

$\begin{array}{llll}\text { A } 87 / 1908 & 37.00 & -1.70 & 1.66\end{array}$

$\begin{array}{llll}\text { A } 87 / 1788 & 37.10 & -1.51 & 1.48\end{array}$

$\begin{array}{llll}\text { A } 87 / 1789 & 37.18 & -1.68 & 1.32\end{array}$

$\begin{array}{llll}\text { A } 87 / 1790 & 37.28 & -1.82 & 1.37\end{array}$

$\begin{array}{llll}\text { A } 87 / 1862 & 37.40 & -1.47 & 1.29\end{array}$

$\begin{array}{llll}\text { A } 87 / 1863 & 37.50 & -1.19 & 1.70\end{array}$

$\begin{array}{llll}\text { A } 87 / 2141 & 37.60 & -1.16 & 1.64\end{array}$

$\begin{array}{llll}\text { A } 87 / 1864 & 37.71 & -1.31 & 1.48\end{array}$

$\begin{array}{llll}\text { A } 87 / 1865 & 37.80 & -1.61 & 1.30\end{array}$

$\begin{array}{llll}\text { A } 87 / 1866 & 37.90 & -1.47 & 1.70\end{array}$

$\begin{array}{llll}\text { A } 87 / 1867 & 38.00 & -1.55 & 1.44\end{array}$

$\begin{array}{llll}\text { A } 87 / 1868 & 38.10 & -1.44 & 1.48\end{array}$

$\begin{array}{llll}\text { A } 87 / 1869 & 38.20 & -1.20 & 1.24\end{array}$

$\begin{array}{llll}\text { A } 87 / 1870 & 38.31 & -1.12 & 1.53\end{array}$

$\begin{array}{llll}\text { A } 87 / 1881 & 38.42 & -1.14 & 1.60\end{array}$

$\begin{array}{llll}\text { A } 87 / 1882 & 38.50 & -1.35 & 1.26\end{array}$

$\begin{array}{llll}\text { A } 87 / 1883 & 38.60 & -1.55 & 1.40\end{array}$

$\begin{array}{llll}\text { A } 87 / 1884 & 38.68 & -1.26 & 1.56\end{array}$

$\begin{array}{llll}\text { A } 87 / 1885 & 38.77 & -1.40 & 1.70\end{array}$

$\begin{array}{llll}\text { A } 87 / 1886 & 38.90 & -1.71 & 1.67\end{array}$

$\begin{array}{llll}\text { A } 87 / 1887 & 39.00 & -1.69 & 1.48\end{array}$

$\begin{array}{llll}\text { A } 87 / 1888 & 39.10 & -1.56 & 1.39\end{array}$

$\begin{array}{llll}\text { A } 87 / 1889 & 39.20 & -1.28 & 1.10\end{array}$

$\begin{array}{llll}\text { A } 87 / 1890 & 39.30 & -1.16 & 1.13\end{array}$

$\begin{array}{llll}\text { A } 87 / 1893 & 39.40 & -1.25 & 0.94\end{array}$

$\begin{array}{llll}\text { A } 87 / 1894 & 39.48 & -1.11 & 1.10\end{array}$

$\begin{array}{llll}\text { A } 87 / 1895 & 39.60 & -1.35 & 0.83\end{array}$

$\begin{array}{llll}\text { A } 87 / 1896 & 39.70 & -1.50 & 0.73\end{array}$

$\begin{array}{llll}\text { A } 87 / 1897 & 39.80 & -1.29 & 0.96\end{array}$

$\begin{array}{llll}\text { A } 87 / 1898 & 39.86 & -1.50 & 0.98\end{array}$

$\begin{array}{llll}\text { A } 87 / 1899 & 40.00 & -1.37 & 0.90\end{array}$

$\begin{array}{llll}\text { A } 87 / 1900 \quad 40.10 & -1.36 & 1.42\end{array}$

$\begin{array}{llll}\text { A } 87 / 1901 & 40.18 & -1.38 & 1.05\end{array}$

$\begin{array}{llll}\text { A } 87 / 1902 & 40.27 & -1.46 & 1.13\end{array}$

$\begin{array}{llll}\text { A } 87 / 1903 & 40.40 & -1.20 & 1.30\end{array}$

$\begin{array}{llll}\text { A } 87 / 1904 & 40.50 & -1.47 & 1.46\end{array}$

$\begin{array}{llll}\text { A } 87 / 1905 & 40.60 & -1.83 & 1.71\end{array}$

$\begin{array}{llll}\text { A } 87 / 1906 & 40.70 & -1.87 & 1.68\end{array}$

$\begin{array}{llll}\text { A } 87 / 1907 & 40.80 & -2.07 & 1.35\end{array}$

$\begin{array}{llll}\text { A } 87 / 1909 & 40.90 & -1.87 & 1.62\end{array}$

$\begin{array}{llll}\text { A } 87 / 1910 & 41.00 & -1.65 & 1.77\end{array}$

$\begin{array}{llll}\text { A } 87 / 1921 & 41.10 & -1.31 & 1.69\end{array}$

$\begin{array}{llll}\text { A } 87 / 1922 & 41.20 & -1.32 & 1.43\end{array}$

$\begin{array}{llll}\text { A } 87 / 1923 & 41.30 & -1.40 & 1.39 \\ \text { A } 87 / 1924 & 41.37 & -1.14 & 1.47\end{array}$
Table 2 (continued).

\begin{tabular}{|c|c|c|c|}
\hline $\begin{array}{l}\text { Sample } \\
\text { code }\end{array}$ & $\begin{array}{l}\text { Depth }^{\mathrm{a}} \\
\text { (mbsf) }\end{array}$ & $\delta^{18} \mathrm{O}$ & $\delta^{13} \mathrm{C}$ \\
\hline \multicolumn{4}{|c|}{ Hole 677A (Cont.) } \\
\hline A $87 / 1925$ & 41.50 & -1.10 & 1.46 \\
\hline A $87 / 1926$ & 41.60 & -1.28 & 1.47 \\
\hline A $87 / 1927$ & 41.68 & -1.42 & 1.24 \\
\hline A $87 / 1928$ & 41.77 & -1.49 & 1.24 \\
\hline A $87 / 1929$ & 41.90 & -1.22 & 1.34 \\
\hline A $87 / 1930$ & 42.00 & -0.98 & 1.35 \\
\hline A $87 / 1941$ & 42.10 & -0.97 & 1.31 \\
\hline A $87 / 1942$ & 42.20 & -1.08 & 1.65 \\
\hline A $87 / 1943$ & 42.30 & -1.41 & 1.48 \\
\hline A $87 / 1944$ & 42.40 & -1.81 & 1.43 \\
\hline A $87 / 1945$ & 42.50 & -1.57 & 1.16 \\
\hline A $87 / 1946$ & 42.60 & -1.26 & 1.10 \\
\hline A $87 / 1947$ & 42.70 & -1.05 & 0.88 \\
\hline A $87 / 1948$ & 42.80 & -1.27 & 0.94 \\
\hline A $87 / 1949$ & 42.86 & -0.99 & 0.95 \\
\hline A $87 / 1950$ & 43.00 & -1.05 & 0.73 \\
\hline A $87 / 1971$ & 43.10 & -0.64 & 0.62 \\
\hline A $87 / 1972$ & 43.18 & -0.59 & 0.86 \\
\hline A $87 / 1973$ & 43.27 & -0.92 & 0.93 \\
\hline A $87 / 1974$ & 43.40 & -0.97 & 1.01 \\
\hline A $87 / 1975$ & 43.50 & -1.15 & 0.76 \\
\hline A $87 / 1976$ & 43.70 & -1.30 & 1.01 \\
\hline A $87 / 1977$ & 43.80 & -1.21 & 1.36 \\
\hline A $87 / 1978$ & 43.90 & -1.48 & 1.12 \\
\hline A $87 / 1979$ & 44.00 & -1.19 & 1.15 \\
\hline A $87 / 1980$ & 44.30 & -1.47 & 1.54 \\
\hline A $87 / 1981$ & 44.40 & -1.59 & 1.65 \\
\hline A $87 / 1982$ & 44.50 & -1.93 & 1.78 \\
\hline A $87 / 1983$ & 44.60 & -2.16 & 1.59 \\
\hline A $87 / 1984$ & 44.70 & -1.84 & 1.60 \\
\hline A $87 / 1985$ & 44.80 & -1.82 & 1.65 \\
\hline A $87 / 1986$ & 44.90 & -1.45 & 1.62 \\
\hline A $87 / 1987$ & 45.02 & -1.35 & 1.51 \\
\hline A $87 / 1988$ & 45.10 & -1.39 & 1.49 \\
\hline A $87 / 1989$ & 45.18 & -1.24 & 1.48 \\
\hline A $87 / 1990$ & 45.30 & -1.57 & 1.42 \\
\hline A $87 / 2031$ & 45.40 & -1.64 & 1.46 \\
\hline A $87 / 2032$ & 45.50 & -1.49 & 1.56 \\
\hline A $87 / 2033$ & 45.60 & -1.57 & 1.46 \\
\hline A $87 / 2034$ & 45.71 & -1.34 & 1.23 \\
\hline A $87 / 2035$ & 45.80 & -1.24 & 1.09 \\
\hline A $87 / 2036$ & 45.90 & -0.94 & 1.40 \\
\hline A $87 / 2037$ & 46.00 & -0.87 & 1.46 \\
\hline A $87 / 2038$ & 46.10 & -1.00 & 1.10 \\
\hline A $87 / 2039$ & 46.20 & -0.93 & 1.20 \\
\hline A $87 / 2040$ & 46.30 & -0.97 & 1.26 \\
\hline A $87 / 2051$ & 46.40 & -1.08 & 1.28 \\
\hline A $87 / 2052$ & 46.50 & -1.18 & 1.05 \\
\hline A $87 / 2053$ & 46.60 & -1.40 & 1.17 \\
\hline A $87 / 2054$ & 46.68 & -1.45 & 1.23 \\
\hline A $87 / 2055$ & 46.80 & -1.01 & 1.44 \\
\hline A $87 / 2056$ & 46.90 & -1.33 & 1.46 \\
\hline A $87 / 2057$ & 47.00 & -1.70 & 1.21 \\
\hline A $87 / 2058$ & 47.10 & -1.51 & 1.56 \\
\hline A $87 / 2059$ & 47.21 & -1.73 & 1.63 \\
\hline A $87 / 2060$ & 47.30 & -1.88 & 1.45 \\
\hline A $87 / 2061$ & 47.40 & -1.66 & 1.59 \\
\hline A $87 / 2062$ & 47.50 & -1.79 & 1.68 \\
\hline A $87 / 2063$ & 47.60 & -1.27 & 1.23 \\
\hline A $87 / 2081$ & 47.60 & -1.19 & 1.29 \\
\hline A $87 / 2064$ & 47.70 & -1.97 & 1.46 \\
\hline A $87 / 2065$ & 47.81 & -1.87 & 1.36 \\
\hline A $87 / 2066$ & 47.90 & -1.57 & 1.01 \\
\hline A $87 / 2067$ & 48.00 & -1.49 & 1.11 \\
\hline A $87 / 2068$ & 48.10 & -1.13 & 0.68 \\
\hline A $87 / 2069$ & 48.20 & -0.62 & 0.84 \\
\hline A $87 / 2070$ & 48.30 & -0.60 & 0.81 \\
\hline A $87 / 2071$ & 48.80 & -1.19 & 1.09 \\
\hline A $87 / 2072$ & 48.90 & -1.20 & 1.22 \\
\hline A $87 / 2073$ & 49.00 & -1.33 & 1.35 \\
\hline A $87 / 2074$ & 49.08 & -1.56 & 1.30 \\
\hline A $87 / 2075$ & 49.19 & -1.10 & 1.36 \\
\hline A $87 / 2076$ & 49.30 & -1.12 & 1.45 \\
\hline A $87 / 2077$ & 49.39 & -1.47 & 1.48 \\
\hline A $87 / 2078$ & 49.49 & -1.50 & 1.47 \\
\hline $87 / 2079$ & 49.59 & -1.61 & 1.51 \\
\hline
\end{tabular}


Table 2 (continued).

\begin{tabular}{cccc}
\hline $\begin{array}{c}\text { Sample } \\
\text { code }\end{array}$ & $\begin{array}{c}\text { Depth } \\
\text { (mbsf) }\end{array}$ & $\delta^{18} \mathrm{O}$ & $\delta^{13} \mathrm{C}$ \\
\hline
\end{tabular}

Hole 677A (Cont.)

\begin{tabular}{|c|c|c|c|}
\hline A $87 / 2080$ & 49.66 & -1.20 & 1.56 \\
\hline A $87 / 2082$ & 49.80 & -1.06 & 0.87 \\
\hline A $87 / 2083$ & 49.90 & -1.08 & 0.95 \\
\hline A $87 / 2084$ & 50.00 & -0.88 & 0.80 \\
\hline A $87 / 2085$ & 50.10 & -0.84 & 0.94 \\
\hline A $87 / 2086$ & 50.21 & -1.01 & 1.06 \\
\hline A $87 / 2087$ & 50.29 & -0.76 & 0.89 \\
\hline A $87 / 2088$ & 50.40 & -0.91 & 1.03 \\
\hline A $87 / 2089$ & 50.50 & -0.80 & 1.14 \\
\hline A $87 / 2090$ & 50.60 & -0.95 & 1.29 \\
\hline A $87 / 2091$ & 50.70 & -1.44 & 1.32 \\
\hline A $87 / 2092$ & 50.80 & -1.59 & 1.38 \\
\hline A $87 / 2093$ & 50.90 & -1.34 & 1.40 \\
\hline A $87 / 2094$ & 50.99 & -1.37 & 1.39 \\
\hline A $87 / 2095$ & 51.10 & -1.34 & 1.34 \\
\hline A $87 / 2096$ & 51.18 & -1.25 & 1.48 \\
\hline A $87 / 2097$ & 51.30 & -1.20 & 1.09 \\
\hline A $87 / 2098$ & 51.40 & -1.41 & 1.01 \\
\hline A $87 / 2099$ & 51.50 & -1.03 & 1.01 \\
\hline A $87 / 2100$ & 51.60 & -1.10 & 0.62 \\
\hline A $87 / 2142$ & 51.80 & -1.39 & 0.82 \\
\hline A $87 / 2143$ & 51.90 & -1.32 & 1.00 \\
\hline A $87 / 2144$ & 52.00 & -1.40 & 0.82 \\
\hline A $87 / 2145$ & 52.10 & -0.83 & 1.34 \\
\hline A $87 / 2146$ & 52.20 & -1.05 & 1.26 \\
\hline A $87 / 2147$ & 52.30 & -0.98 & 1.29 \\
\hline A $87 / 2148$ & 52.40 & -1.38 & 0.91 \\
\hline A $87 / 2149$ & 52.50 & -1.55 & 1.51 \\
\hline A $87 / 2150$ & 52.61 & -1.65 & 1.34 \\
\hline A $87 / 2151$ & 52.67 & -1.64 & 1.55 \\
\hline A $87 / 2152$ & 52.80 & -1.76 & 1.42 \\
\hline A $87 / 2153$ & 52.90 & -1.63 & 1.55 \\
\hline A $87 / 2154$ & 53.00 & -1.78 & 1.21 \\
\hline A $87 / 2155$ & 53.10 & -1.48 & 1.16 \\
\hline A $87 / 2156$ & 53.30 & -0.96 & 0.96 \\
\hline A $87 / 2157$ & 53.40 & -0.98 & 0.85 \\
\hline A $87 / 2158$ & 53.48 & -0.99 & 1.06 \\
\hline A $87 / 2159$ & 53.60 & -1.29 & 1.07 \\
\hline A $87 / 2160$ & 53.70 & -1.15 & 0.87 \\
\hline A $87 / 2161$ & 53.80 & -1.14 & 1.08 \\
\hline A $87 / 2177$ & 53.81 & -1.67 & 1.32 \\
\hline A $87 / 2178$ & 53.90 & -1.59 & 1.43 \\
\hline A $87 / 2179$ & 54.00 & -1.76 & 1.58 \\
\hline A $87 / 2180$ & 54.10 & -1.43 & 1.38 \\
\hline A $87 / 2181$ & 54.20 & -1.60 & 1.35 \\
\hline A $87 / 2182$ & 54.30 & -1.74 & 1.19 \\
\hline A $87 / 2183$ & 54.40 & -1.36 & 1.18 \\
\hline A $87 / 2184$ & 54.50 & -1.40 & 0.95 \\
\hline A $87 / 2185$ & 54.60 & -1.27 & 1.23 \\
\hline A $87 / 2186$ & 54.68 & -1.20 & 1.15 \\
\hline A $87 / 2187$ & 54.80 & -1.11 & 0.95 \\
\hline A $87 / 2188$ & 54.90 & -1.39 & 0.89 \\
\hline A $87 / 2189$ & 55.00 & -1 & 1.03 \\
\hline A $87 / 2190$ & 55.10 & -1.15 & 0.74 \\
\hline A $87 / 2191$ & 55.20 & -1.06 & 0.66 \\
\hline A $87 / 2192$ & 55.30 & -1.11 & 0.52 \\
\hline A $87 / 2193$ & 55.40 & -1.17 & 0.60 \\
\hline A $87 / 2194$ & 55.50 & -1.32 & 0.87 \\
\hline A $87 / 2195$ & 55.60 & -1.45 & 0.87 \\
\hline A $87 / 2196$ & 55.70 & -1.28 & 0.89 \\
\hline A $87 / 2197$ & 55.80 & -1.28 & 0.77 \\
\hline A $87 / 2198$ & 55.90 & -1.45 & 0.88 \\
\hline A $87 / 2199$ & 56.00 & -1.73 & 1.53 \\
\hline A $87 / 2200$ & 56.10 & -1.65 & 1.63 \\
\hline A $87 / 2201$ & 56.18 & -1.70 & 1.36 \\
\hline A $87 / 2202$ & 56.30 & -1.79 & 1.42 \\
\hline A $87 / 2203$ & 56.40 & -1.73 & 1.46 \\
\hline A $87 / 2211$ & 56.50 & -1.86 & 1.49 \\
\hline A $87 / 2212$ & 56.60 & -1.75 & 1.42 \\
\hline A $87 / 2213$ & 56.70 & -1.96 & 1.26 \\
\hline A $87 / 2214$ & 56.80 & -1.80 & 0.91 \\
\hline A $87 / 2215$ & 56.90 & -1.55 & 0.75 \\
\hline A $87 / 2216$ & 57.00 & -1.22 & 0.83 \\
\hline A $87 / 2217$ & 57.10 & -1.21 & 0.65 \\
\hline A $87 / 2218$ & 57.20 & -0.99 & 0.81 \\
\hline A $87 / 2219$ & 57.30 & -1.07 & 0.84 \\
\hline
\end{tabular}

Table 2 (continued).

\begin{tabular}{cccc}
\hline $\begin{array}{c}\text { Sample } \\
\text { code }\end{array}$ & $\begin{array}{l}\text { Depth } \\
\text { (mbsf) }\end{array}$ & $\delta^{18} \mathrm{O}$ & $\delta^{13} \mathrm{C}$ \\
\hline
\end{tabular}

Hole 677A (Cont.)

\begin{tabular}{|c|c|c|c|}
\hline A $87 / 2220$ & 57.39 & -1.00 & 1.11 \\
\hline A $87 / 2221$ & 57.50 & -1.19 & 0.96 \\
\hline A $87 / 2223$ & 57.60 & -1.07 & 0.93 \\
\hline A $87 / 2224$ & 57.68 & -1.73 & 1.38 \\
\hline A $87 / 2225$ & 57.80 & -1.90 & 1.39 \\
\hline A $87 / 2226$ & 57.90 & -1.85 & 1.38 \\
\hline A $87 / 2275$ & 58.00 & -1.86 & 1.43 \\
\hline A $87 / 2227$ & 58.10 & -1.64 & 1.43 \\
\hline A $87 / 2228$ & 58.20 & -1.62 & 1.34 \\
\hline A $87 / 2229$ & 58.30 & -1.58 & 1.72 \\
\hline A $87 / 2230$ & 58.40 & -1.72 & 1.62 \\
\hline A $87 / 2231$ & 58.50 & -1.90 & 1.22 \\
\hline A $87 / 2232$ & 58.60 & -1.34 & 0.90 \\
\hline A $87 / 2233$ & 58.70 & -1.05 & 0.88 \\
\hline A $87 / 2234$ & 58.80 & -1.15 & 1.11 \\
\hline A $87 / 2235$ & 58.90 & -1.36 & 0.77 \\
\hline A $87 / 2236$ & 59.00 & -1.21 & 0.99 \\
\hline A $87 / 2237$ & 59.10 & -1.07 & 0.99 \\
\hline A $87 / 2238$ & 59.18 & -1.23 & 1.16 \\
\hline A $87 / 2239$ & 59.30 & -1.45 & 1.39 \\
\hline A $87 / 2240$ & 59.40 & -1.46 & 1.44 \\
\hline A $87 / 2241$ & 59.50 & -1.53 & 1.22 \\
\hline A $87 / 2242$ & 59.60 & -1.43 & 1.23 \\
\hline A $87 / 2243$ & 59.70 & -1.41 & 1.03 \\
\hline A $87 / 2244$ & 59.80 & -1.26 & 0.86 \\
\hline A $87 / 2245$ & 59.90 & -1.07 & 0.79 \\
\hline A $87 / 2246$ & 60.00 & -1.29 & 0.81 \\
\hline A $87 / 2247$ & 60.10 & -1.01 & 0.81 \\
\hline A $87 / 2248$ & 60.20 & -0.84 & 0.53 \\
\hline A $87 / 2249$ & 60.30 & -0.84 & 0.81 \\
\hline A $87 / 2250$ & 60.40 & -1.17 & 0.72 \\
\hline A $87 / 2251$ & 60.50 & -1.40 & 1.08 \\
\hline A $87 / 2252$ & 60.60 & -1.55 & 0.96 \\
\hline A $87 / 2253$ & 60.68 & -1.40 & 1.37 \\
\hline A $87 / 2254$ & 60.80 & -1.48 & 1.27 \\
\hline A $87 / 2255$ & 60.90 & -1.77 & 1.30 \\
\hline A $87 / 2256$ & 61.00 & -1.51 & 1.34 \\
\hline A $87 / 2257$ & 61.10 & -1.82 & 1.16 \\
\hline A $87 / 2258$ & 61.20 & -1.83 & 1.38 \\
\hline A $87 / 2259$ & 61.30 & -1.70 & 1.51 \\
\hline A $87 / 2260$ & 61.40 & -1.52 & 1.15 \\
\hline A $87 / 2261$ & 61.50 & -1.59 & 1.05 \\
\hline A $87 / 2262$ & 61.60 & -1.50 & 0.79 \\
\hline A $87 / 2263$ & 61.70 & -1.02 & 0.89 \\
\hline A $87 / 2264$ & 61.80 & -1.31 & 1.04 \\
\hline A $87 / 2265$ & 61.90 & -1.30 & 1.12 \\
\hline A $87 / 2266$ & 62.00 & -1.49 & 0.94 \\
\hline A $87 / 2267$ & 62.10 & -1 & 0.99 \\
\hline A $87 / 2268$ & 62.18 & -1.47 & 1.34 \\
\hline A $87 / 2269$ & 62.30 & -1.73 & 1.17 \\
\hline A $87 / 2270$ & 62.40 & -1.56 & 1.30 \\
\hline A $87 / 2271$ & 62.50 & -1.75 & 1.42 \\
\hline A $87 / 2272$ & 62.60 & -1.86 & 1.63 \\
\hline A $87 / 2273$ & 62.70 & -1.65 & 1.61 \\
\hline A $87 / 2274$ & 62.80 & -1.52 & 1.47 \\
\hline A $87 / 2276$ & 62.90 & -1.75 & 1.40 \\
\hline A $87 / 2277$ & 63.00 & -1.68 & 1.28 \\
\hline A $87 / 2278$ & 63.10 & -1.71 & 1.43 \\
\hline A $87 / 2279$ & 63.18 & -1.38 & 1.35 \\
\hline A $88 / 91$ & 63.30 & -0.97 & 0.89 \\
\hline A $88 / 92$ & 63.40 & - & 0.82 \\
\hline A $87 / 93$ & 63.50 & -1.08 & 0.84 \\
\hline A $88 / 94$ & 63.60 & -1.09 & 0.79 \\
\hline A $88 / 95$ & 63.70 & -0.92 & 1.23 \\
\hline A $88 / 96$ & 63.80 & -0.72 & 0.86 \\
\hline A $88 / 97$ & 63.90 & -0.98 & 0.63 \\
\hline A $88 / 98$ & 64.00 & -1.07 & 1.00 \\
\hline A $88 / 99$ & 64.10 & -1.14 & 1.23 \\
\hline A $88 / 100$ & 64.18 & -1.41 & 1.17 \\
\hline A $88 / 101$ & 64.30 & -1.28 & 1.17 \\
\hline A $88 / 102$ & 64.40 & -0.95 & 0.76 \\
\hline A $88 / 141$ & 64.40 & -1.23 & 1.15 \\
\hline A $88 / 103$ & 64.50 & -1.15 & 0.95 \\
\hline A $88 / 104$ & 64.60 & -1.15 & 0.93 \\
\hline A $88 / 105$ & 64.70 & -1.24 & 0.85 \\
\hline A $88 / 106$ & 64.80 & -1.27 & 1.13 \\
\hline
\end{tabular}

Table 2 (continued).

\begin{tabular}{|c|c|c|c|}
\hline $\begin{array}{l}\text { Sample } \\
\text { code }\end{array}$ & $\begin{array}{l}\text { Depth } \\
\text { (mbsf) }\end{array}$ & $\delta^{18} \mathrm{O}$ & $\delta^{13} \mathrm{C}$ \\
\hline \multicolumn{4}{|c|}{ Hole 677A (Cont.) } \\
\hline A $88 / 107$ & 64.90 & -1.24 & 0.83 \\
\hline A $88 / 108$ & 65.00 & -1.39 & 0.83 \\
\hline A $88 / 109$ & 65.10 & -1.19 & 0.59 \\
\hline A $88 / 110$ & 65.20 & -1.02 & 0.81 \\
\hline A $88 / 111$ & 65.30 & -0.95 & 0.69 \\
\hline A $88 / 112$ & 65.41 & -0.33 & -0.03 \\
\hline A $88 / 113$ & 65.50 & -1.48 & 1.02 \\
\hline A $88 / 114$ & 65.68 & -1.00 & 1.13 \\
\hline A $88 / 115$ & 65.80 & -1.36 & 1.17 \\
\hline A $88 / 116$ & 65.90 & -1.57 & 0.88 \\
\hline A $88 / 117$ & 66.00 & -1.50 & 0.85 \\
\hline A $88 / 118$ & 66.10 & -1.45 & 0.99 \\
\hline A $88 / 119$ & 66.20 & -1.21 & 0.94 \\
\hline A $88 / 120$ & 66.31 & -1.23 & 1.22 \\
\hline A $88 / 121$ & 66.40 & -1.29 & 1.20 \\
\hline A $88 / 122$ & 66.50 & -1.45 & 1.06 \\
\hline A $88 / 123$ & 66.60 & -1.70 & 1.23 \\
\hline A $88 / 274$ & 66.60 & -1.71 & 1.08 \\
\hline A $88 / 124$ & 66.80 & -1.29 & 1.10 \\
\hline A $88 / 125$ & 66.90 & -1.20 & 0.79 \\
\hline A $88 / 126$ & 67.00 & -0.99 & 0.97 \\
\hline A $88 / 127$ & 67.10 & -1.26 & 0.68 \\
\hline A $88 / 128$ & 67.18 & -0.99 & 1.15 \\
\hline A $88 / 129$ & 67.30 & -1.50 & 1.03 \\
\hline A $88 / 130$ & 67.40 & -1.79 & 1.10 \\
\hline A $88 / 131$ & 67.50 & -1.73 & 1.01 \\
\hline A $88 / 132$ & 67.60 & -1.55 & 1.41 \\
\hline A $88 / 351$ & 67.70 & -1.70 & 1.05 \\
\hline A $88 / 134$ & 67.80 & -1.59 & 1.39 \\
\hline A $88 / 135$ & 67.90 & -1.45 & 1.20 \\
\hline A $88 / 136$ & 68.00 & -1.36 & 1.23 \\
\hline A $88 / 137$ & 68.10 & -1.12 & 0.87 \\
\hline A $88 / 138$ & 68.20 & -1.15 & 0.59 \\
\hline A $88 / 139$ & 68.30 & -1.12 & 0.62 \\
\hline A $88 / 140$ & 68.40 & -1.19 & 1.27 \\
\hline A $88 / 142$ & 68.50 & -0.86 & 0.25 \\
\hline A $88 / 143$ & 68.60 & -1.22 & 0.49 \\
\hline A $88 / 144$ & 68.68 & -1.14 & 0.27 \\
\hline A $88 / 145$ & 68.80 & -1.14 & 0.87 \\
\hline A $88 / 146$ & 68.90 & -0.72 & 0.83 \\
\hline A $88 / 147$ & 69.00 & -0.74 & 0.69 \\
\hline A $88 / 148$ & 69.10 & -0.72 & 0.52 \\
\hline A $88 / 149$ & 69.20 & -0.88 & 0.76 \\
\hline A $88 / 150$ & 69.30 & -1.19 & 0.69 \\
\hline A $88 / 271$ & 69.40 & -1.11 & 0.72 \\
\hline A $88 / 272$ & 69.50 & -1.13 & 0.78 \\
\hline A $88 / 273$ & 69.60 & -1.24 & 0.84 \\
\hline A $88 / 275$ & 69.70 & -1.36 & 1.03 \\
\hline A $88 / 276$ & 69.80 & -1.47 & 1.15 \\
\hline A $88 / 277$ & 69.90 & -1.71 & 1.32 \\
\hline A $88 / 278$ & 70.00 & -1.53 & 1.35 \\
\hline A $88 / 279$ & 70.14 & -1.54 & 1.29 \\
\hline A $88 / 349$ & 70.30 & -1.34 & 1.02 \\
\hline A $88 / 350$ & 70.40 & -1.51 & 1.20 \\
\hline A $88 / 352$ & 70.50 & -1.72 & 1.02 \\
\hline A $88 / 353$ & 70.60 & -1.76 & 1.12 \\
\hline A $88 / 354$ & 70.70 & -1.47 & 0.86 \\
\hline A $88 / 355$ & 70.80 & -1.06 & 0.58 \\
\hline A $88 / 356$ & 70.90 & -1.21 & 0.61 \\
\hline A $88 / 357$ & 71.00 & -0.93 & 0.84 \\
\hline A $88 / 358$ & 71.10 & -1.18 & 0.98 \\
\hline A $88 / 359$ & 71.20 & -1.09 & 1.03 \\
\hline A $88 / 360$ & 71.30 & -1.10 & 1.05 \\
\hline A $88 / 361$ & 71.40 & -1.49 & 1.27 \\
\hline A $88 / 362$ & 71.50 & -1.67 & 1.13 \\
\hline A $88 / 363$ & 71.60 & -1.57 & 1.02 \\
\hline A $88 / 364$ & 71.68 & -1.43 & 1.11 \\
\hline A $88 / 365$ & 71.80 & -1.29 & 1.03 \\
\hline A $88 / 366$ & 71.90 & -1.43 & 1.05 \\
\hline A $88 / 367$ & 72.00 & -1.06 & 1.11 \\
\hline A $88 / 368$ & 72.10 & -1.17 & 0.95 \\
\hline A $88 / 369$ & 72.20 & -1.49 & 1.34 \\
\hline A $88 / 331$ & 72.30 & -1.64 & 1.30 \\
\hline A $88 / 332$ & 72.40 & -1.67 & 1.33 \\
\hline A $88 / 333$ & 72.50 & -1.84 & 1.24 \\
\hline A $88 / 334$ & 72.70 & -1.58 & 1.04 \\
\hline
\end{tabular}


Table 2 (continued).

\begin{tabular}{cccc}
\hline $\begin{array}{c}\text { Sample } \\
\text { code }\end{array}$ & $\begin{array}{l}\text { Depth } \\
\text { (mbsf) }\end{array} \quad \delta^{18} \mathrm{O}$ & $\delta^{13} \mathrm{C}$
\end{tabular}

Hole 677A (Cont.)

\begin{tabular}{|c|c|c|c|}
\hline A $88 / 335$ & 72.80 & -1.38 & 1.17 \\
\hline A $88 / 336$ & 72.90 & -1.45 & 1.13 \\
\hline A $88 / 337$ & 73.00 & -1.47 & 1.16 \\
\hline A $88 / 338$ & 73.10 & -1.43 & 1.10 \\
\hline A $88 / 339$ & 73.20 & -1.38 & 1.39 \\
\hline A $88 / 340$ & 73.28 & -1.30 & 0.89 \\
\hline A $88 / 341$ & 73.40 & -1.53 & 1.16 \\
\hline A $88 / 342$ & 73.49 & -1.37 & 0.96 \\
\hline A $88 / 343$ & 73.59 & -1.57 & 0.84 \\
\hline A $88 / 344$ & 73.68 & -1.70 & 0.8 \\
\hline A $88 / 345$ & 73.79 & -1.45 & 0.87 \\
\hline A $88 / 346$ & 73.89 & -1.37 & 0.53 \\
\hline A $88 / 347$ & 74.00 & -1.51 & 0.75 \\
\hline A $88 / 348$ & 74.09 & -1.02 & 0.37 \\
\hline A $88 / 370$ & 74.20 & -1.31 & 0.69 \\
\hline A $88 / 371$ & 74.30 & -1.18 & 0.47 \\
\hline A $88 / 372$ & 74.38 & -1.37 & 0.66 \\
\hline A $88 / 373$ & 74.48 & -1.38 & 0.83 \\
\hline A $88 / 374$ & 74.60 & -1.26 & 1.06 \\
\hline A $88 / 375$ & 74.70 & -1.69 & 1.05 \\
\hline A $88 / 376$ & 74.78 & -1.49 & 1.24 \\
\hline A $88 / 377$ & 74.90 & -1.89 & 1.14 \\
\hline A $88 / 378$ & 75.00 & -1.35 & 1.56 \\
\hline A $88 / 380$ & 75.18 & -1.61 & 1.27 \\
\hline A $88 / 582$ & 75.40 & -1.52 & 1.02 \\
\hline A $88 / 583$ & 75.50 & -1.65 & 1.28 \\
\hline A $88 / 584$ & 75.60 & -1.67 & 0.98 \\
\hline A $88 / 585$ & 75.70 & -1.57 & 0.89 \\
\hline A $88 / 586$ & 75.80 & -1.44 & 0.78 \\
\hline A $88 / 587$ & 75.89 & -1.59 & 1.07 \\
\hline A $88 / 588$ & 76.00 & -1.35 & 0.97 \\
\hline A $88 / 589$ & 76.10 & -0.80 & 0.89 \\
\hline A $88 / 590$ & 76.20 & -1.15 & 1.27 \\
\hline A $88 / 591$ & 76.27 & -1.26 & 1.11 \\
\hline A $88 / 592$ & 76.40 & -1.31 & 1.38 \\
\hline A $88 / 593$ & 76.50 & -1.21 & 1.52 \\
\hline A $88 / 594$ & 76.60 & -1.21 & 1.51 \\
\hline A $88 / 595$ & 76.70 & -1.40 & 1.04 \\
\hline A $88 / 596$ & 76.81 & -1.71 & 1.58 \\
\hline A $88 / 597$ & 77.20 & -1.38 & 1.27 \\
\hline A $88 / 598$ & 77.30 & -1.60 & 1.34 \\
\hline A $88 / 599$ & 77.39 & -1.49 & 1.46 \\
\hline A $88 / 600$ & 77.50 & -1.48 & 1.09 \\
\hline A $88 / 601$ & 77.60 & -1.67 & 1.11 \\
\hline A $88 / 602$ & 77.70 & -1.69 & 1.39 \\
\hline A $88 / 603$ & 77.78 & -1.47 & 1.26 \\
\hline A $88 / 604$ & 77.90 & -1.48 & 1.62 \\
\hline A $88 / 605$ & 78.00 & -1.33 & 1.33 \\
\hline A $88 / 606$ & 78.10 & -1.49 & 1.20 \\
\hline A $88 / 607$ & 78.18 & -1.39 & 1.28 \\
\hline A $88 / 608$ & 78.30 & -1.60 & 1.15 \\
\hline A $88 / 609$ & 78.40 & -1.29 & 1.36 \\
\hline A $88 / 610$ & 78.50 & -1.29 & 1.41 \\
\hline A $88 / 611$ & 78.60 & -1.32 & 1.18 \\
\hline A $88 / 612$ & 78.70 & -1.14 & 1.20 \\
\hline A $88 / 613$ & 78.80 & -1.27 & 1.28 \\
\hline A $88 / 614$ & 78.89 & -1.14 & 1.19 \\
\hline A $88 / 615$ & 79.00 & -1.30 & 1.09 \\
\hline A $88 / 616$ & 79.10 & -1.14 & 1.29 \\
\hline A $88 / 617$ & 79.20 & -1.21 & 1.00 \\
\hline A $88 / 618$ & 79.30 & -1.29 & 0.96 \\
\hline A $88 / 619$ & 79.40 & -1.21 & 1.18 \\
\hline A $88 / 620$ & 79.50 & -1.32 & 1.10 \\
\hline A $88 / 711$ & 79.60 & -1.15 & 1.09 \\
\hline A $88 / 712$ & 79.68 & -0.94 & 1.01 \\
\hline A $88 / 713$ & 79.80 & -1.37 & 1.08 \\
\hline A $88 / 714$ & 79.90 & -1.06 & 0.95 \\
\hline A $88 / 715$ & 80.01 & -1.17 & 1.03 \\
\hline A $88 / 716$ & 80.10 & -1.27 & 0.95 \\
\hline A $88 / 717$ & 80.20 & -1.28 & 1.23 \\
\hline A $88 / 718$ & 80.29 & -1.26 & 0.99 \\
\hline A $88 / 719$ & 80.39 & -1.37 & 1.02 \\
\hline A $88 / 720$ & 80.49 & -1.16 & 0.79 \\
\hline A $88 / 872$ & 80.59 & -1.32 & 1.18 \\
\hline A $88 / 873$ & 80.69 & -1.30 & 1.27 \\
\hline A $88 / 874$ & 80.79 & -1.15 & 1.25 \\
\hline
\end{tabular}

Table 2 (continued)

\begin{tabular}{cccc}
\hline $\begin{array}{c}\text { Sample } \\
\text { code }\end{array}$ & $\begin{array}{l}\text { Depth }^{\mathrm{a}} \\
\text { (mbsf) }\end{array}$ & $\delta^{18} \mathrm{O}$ & $\delta^{13} \mathrm{C}$ \\
\hline
\end{tabular}

Hole 677A (Cont.)

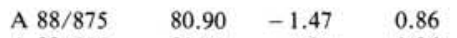

$\begin{array}{llll}\text { A } 88 / 876 & 81.00 & -1.47 & 0.86\end{array}$

$\begin{array}{llll}\text { A } 88 / 877 & 81.10 & -1.30 & 0.93\end{array}$

$\begin{array}{llll}\text { A } 88 / 878 & 81.18 & -1.28 & 0.93\end{array}$

$\begin{array}{llll}\text { A } 88 / 879 & 81.30 & -1.39 & 0.83\end{array}$

$\begin{array}{llll}\text { A } 88 / 880 & 81.41 & -1.20 & 0.85\end{array}$

$\begin{array}{llll}\text { A } 88 / 881 & 81.50 & -1.27 & 0.74 \\ \text { A } 88 / 882 & 81.60 & -1.36 & 0.90\end{array}$

$\begin{array}{llll}\text { A } 88 / 882 & 81.60 & -1.36 & 0.90 \\ \text { A } 88 / 883 & 82.20 & -1.31 & 1.08\end{array}$

A $88 / 884 \quad 82.30 \quad-1.27 \quad 1.15$

$\begin{array}{llll}\text { A } 88 / 885 & 82.39 & -1.30 & 0.89\end{array}$

$\begin{array}{llll}\text { A } 88 / 886 & 82.50 & -1.21 & 1.04 \\ \text { A } 88 / 887 & 82.60 & -1.58 & 0.65\end{array}$

$\begin{array}{llll}\text { A } 88 / 888 & 82.70 & -1.45 & 0.79\end{array}$

$\begin{array}{llll}\text { A } 88 / 889 & 82.80 & -1.42 & 0.78 \\ \text { A } 88 / 890 & 82.90 & -0.89 & 1.08\end{array}$

$\begin{array}{llll}\text { A } 88 / 890 & 82.90 & -0.89 & 1.08 \\ \text { A } 88 / 891 & 83.00 & -1.33 & 0.61\end{array}$

$\begin{array}{llll}\text { A } 88 / 892 & 83.10 & -1.56 & 0.41\end{array}$

$\begin{array}{llll}\text { A } 88 / 893 & 83.18 & -1.12 & 0.83\end{array}$

$\begin{array}{llll}\text { A } 88 / 894 & 83.30 & -1.76 & 0.53\end{array}$

$\begin{array}{llll}\text { A } 88 / 895 & 83.40 & -1.22 & 0.68\end{array}$

$\begin{array}{llll}\text { A } 88 / 902 & 83.50 & -1.31 & 0.44\end{array}$

$\begin{array}{llll}\text { A } 88 / 896 & 83.60 & -1.27 & 1.03\end{array}$

$\begin{array}{llll}\text { A } 88 / 897 & 83.70 & -1.35 & 0.68\end{array}$

$\begin{array}{llll}\text { A } 88 / 898 & 83.80 & -1.50 & 0.82\end{array}$

$\begin{array}{llll}\text { A } 88 / 927 & 83.80 & -1.60 & 0.29\end{array}$

$\begin{array}{llll}\text { A } 88 / 899 & 83.89 & -0.71 & 0.35 \\ \text { A } 88 / 928 & 83.89 & -0.89 & 0.58\end{array}$

$\begin{array}{llll}\text { A } 88 / 928 & 83.89 & -0.89 & 0.58\end{array}$

$\begin{array}{llll}\text { A } 88 / 900 & 84.00 & -1.64 & 0.58 \\ \text { A } 88 / 929 & 84.00 & -1.82 & 0.64\end{array}$

$\begin{array}{llll}\text { A } 88 / 929 & 84.00 & -1.82 & 0.64 \\ \text { A } 88 / 901 & 84.10 & -0.98 & 0.80\end{array}$

A $88 / 903 \quad 84.20 \quad-1.72 \quad 0.85$

$\begin{array}{llll}\text { A } 88 / 904 & 84.29 & -1.45 & 0.80\end{array}$

$\begin{array}{llll}\text { A } 88 / 905 & 84.40 & -0.93 & 0.79\end{array}$

$\begin{array}{llll}\text { A } 88 / 906 & 84.50 & -1.58 & 0.35\end{array}$

$\begin{array}{llll}\text { A } 88 / 907 & 84.60 & -1.79 & 1.05\end{array}$

$\begin{array}{llll}\text { A } 88 / 908 & 84.68 & -1.66 & 0.90\end{array}$

$\begin{array}{llll}\text { A } 88 / 909 & 84.79 & -1.52 & 1.16\end{array}$

$\begin{array}{llll}\text { A } 88 / 910 & 84.89 & -1.73 & 1.03\end{array}$

$\begin{array}{llll}\text { A } 88 / 930 & 85.00 & -1.82 & 1.08\end{array}$

$\begin{array}{llll}\text { A } 88 / 931 & 85.10 & -1.82 & 0.67\end{array}$

$\begin{array}{llll}\text { A } 88 / 932 & 85.20 & -1.65 & 1.00\end{array}$

$\begin{array}{llll}\text { A } 88 / 933 & 85.30 & -1.69 & 1.27\end{array}$

$\begin{array}{llll}\text { A } 88 / 934 & 85.39 & -1.67 & 1.12\end{array}$

$\begin{array}{llll}\text { A } 88 / 935 & 85.50 & -1.54 & 1.21\end{array}$

$\begin{array}{llll}\text { A } 88 / 936 & 85.60 & -1.72 & 1.21\end{array}$

$\begin{array}{llll}\text { A } 88 / 1171 & 85.70 & -1.71 & 1.29\end{array}$

$\begin{array}{llll}\text { A } 88 / 938 & 85.79 & -1.82 & 1.00\end{array}$

$\begin{array}{llll}\text { A } 88 / 939 & 85.90 & -1.57 & 1.08\end{array}$

$\begin{array}{llll}\text { A } 88 / 940 & 86.00 & -1.81 & 1.39\end{array}$

$\begin{array}{llll}\text { A } 88 / 941 & 86.10 & -1.95 & 1.02\end{array}$

A $88 / 942 \quad 86.18 \quad-1.64 \quad 1.40$

$\begin{array}{llll}\text { A } 88 / 943 & 86.30 & -1.54 & 0.67\end{array}$

$\begin{array}{llll}\text { A } 88 / 944 & 86.40 & -1.77 & 0.87\end{array}$

$\begin{array}{llll}\text { A } 88 / 945 & 86.50 & -1.55 & 1.10\end{array}$

A $88 / 946 \quad 86.60 \quad-1.36 \quad 1.27$

$\begin{array}{llll}\text { A } 88 / 947 & 86.70 & -1.59 & 1.28\end{array}$

$\begin{array}{llll}\text { A } 88 / 948 & 86.80 & -1.96 & 1.25\end{array}$

A $88 / 949 \quad 86.89 \quad-1.78 \quad 1.41$

$\begin{array}{llll}\text { A } 88 / 950 & 87.00 & -1.81 & 1.08\end{array}$

$\begin{array}{llll}\text { A } 88 / 961 & 87.10 & -1.73 & 1.41\end{array}$

$\begin{array}{llll}\text { A } 88 / 962 & 87.20 & -1.58 & 1.20\end{array}$

$\begin{array}{llll}\text { A } 88 / 963 & 87.29 & -1.35 & 0.93\end{array}$

$\begin{array}{llll}\text { A } 88 / 964 & 87.39 & -1.36 & 0.84\end{array}$

$\begin{array}{llll}\text { A } 88 / 965 & 87.50 & -1.15 & 0.61\end{array}$

$\begin{array}{llll}\text { A } 88 / 966 & 87.60 & -1.18 & 0.71\end{array}$

$\begin{array}{llll}\text { A } 88 / 967 & 87.68 & -0.67 & 0.59\end{array}$

$\begin{array}{llll}\text { A } 88 / 968 & 87.80 & -1.30 & 0.78\end{array}$

$\begin{array}{llll}\text { A } 88 / 969 & 87.90 & -1.11 & 0.92\end{array}$

$\begin{array}{llll}\text { A } 88 / 970 & 88.00 & -1.31 & 0.84\end{array}$

$\begin{array}{llll}\text { A } 88 / 971 & 88.10 & -1.42 & 1.29\end{array}$

$\begin{array}{llll}\text { A } 88 / 972 & 88.20 & -1.50 & 1.12\end{array}$

$\begin{array}{llll}\text { A } 88 / 973 & 88.30 & -1.52 & 1.02\end{array}$

A $88 / 974 \quad 88.39 \quad-1.33 \quad 1.19$

$\begin{array}{llll}\text { A } 88 / 975 & 88.50 & -1.43 & 1.56 \\ \text { A } 88 / 976 & 88.60 & -1.49 & 1.49\end{array}$
Table 2 (continued).

\begin{tabular}{cccc}
\hline $\begin{array}{c}\text { Sample } \\
\text { code }\end{array}$ & $\begin{array}{l}\text { Depth }^{\mathrm{a}} \\
\text { (mbsf) }\end{array}$ & $\delta^{18} \mathrm{O}$ & $\delta^{13} \mathrm{C}$ \\
\hline
\end{tabular}

Hole 677A (Cont.)

\begin{tabular}{|c|c|c|c|}
\hline A $88 / 977$ & 88.70 & -1.67 & 1.48 \\
\hline A $88 / 978$ & 88.79 & -1.59 & 1.49 \\
\hline A $88 / 979$ & 88.90 & -1.78 & 1.30 \\
\hline A $88 / 980$ & 89.00 & -1.83 & 1.20 \\
\hline A $88 / 981$ & 89.10 & -1.54 & 1.28 \\
\hline A $88 / 982$ & 89.18 & -1.79 & 1.19 \\
\hline A $88 / 983$ & 89.30 & -1.36 & 1.10 \\
\hline A $88 / 984$ & 89.40 & -1.28 & 1.18 \\
\hline A $88 / 985$ & 89.50 & -1.47 & 1.23 \\
\hline A $88 / 986$ & 89.60 & -1.52 & 1.25 \\
\hline A $88 / 987$ & 89.70 & -1.90 & 1.01 \\
\hline A $88 / 988$ & 89.80 & -1.51 & 1.45 \\
\hline A $88 / 989$ & 89.89 & -1.90 & 1.15 \\
\hline A $88 / 990$ & 90.00 & -1.92 & 1.45 \\
\hline A $88 / 1151$ & 90.10 & -1.66 & 1.33 \\
\hline A $88 / 1152$ & 90.20 & -1.65 & 1.09 \\
\hline A $88 / 1153$ & 90.29 & -1.63 & 1.03 \\
\hline A $88 / 1154$ & 90.40 & -1.88 & 1.13 \\
\hline A $88 / 1155$ & 90.50 & -1.63 & 1.13 \\
\hline A $88 / 1156$ & 90.60 & -1.63 & 1.24 \\
\hline A $88 / 1157$ & 90.67 & -1.47 & 1.24 \\
\hline A $88 / 1158$ & 90.80 & -1.64 & 1.08 \\
\hline A $88 / 1159$ & 90.90 & -1.90 & 0.98 \\
\hline A $88 / 1160$ & 91.00 & -1.75 & 1.23 \\
\hline A $88 / 1161$ & 91.10 & -1.57 & 1.22 \\
\hline A $88 / 1162$ & 91.20 & -1.69 & 0.99 \\
\hline A $88 / 1163$ & 91.30 & -1.47 & 0.74 \\
\hline A $88 / 1164$ & 91.39 & -1.47 & 0.81 \\
\hline A $88 / 1165$ & 91.50 & -1.51 & 0.69 \\
\hline A $88 / 1166$ & 91.60 & -1.20 & 0.75 \\
\hline A $88 / 1167$ & 91.70 & -1.42 & 0.68 \\
\hline A $88 / 1168$ & 91.71 & -1.60 & 1.08 \\
\hline A $88 / 1169$ & 91.80 & -1.48 & 0.98 \\
\hline A $88 / 1170$ & 91.90 & -1.45 & 0.94 \\
\hline A $88 / 1172$ & 92.00 & -1.57 & 0.88 \\
\hline A $88 / 1173$ & 92.10 & -1.56 & 0.99 \\
\hline A $88 / 1174$ & 92.20 & -1.54 & 1.10 \\
\hline A $88 / 1175$ & 92.30 & -1.45 & 1.35 \\
\hline A $88 / 1176$ & 92.40 & -1.84 & 1.46 \\
\hline A $88 / 1177$ & 92.50 & -2.04 & 1.35 \\
\hline A $88 / 1178$ & 92.60 & -1.73 & 1.33 \\
\hline A $88 / 1179$ & 92.68 & -1.83 & 1.04 \\
\hline A $88 / 1180$ & 92.80 & -1.68 & 1.16 \\
\hline A $88 / 1181$ & 92.90 & -1.80 & 1.10 \\
\hline A $88 / 1182$ & 93.00 & -1.75 & 1.07 \\
\hline A $88 / 1181$ & 93.10 & -1.52 & 1.23 \\
\hline A $88 / 1184$ & 93.20 & -1.42 & 1.07 \\
\hline A $88 / 1185$ & 93.30 & -1.59 & 1.07 \\
\hline A $88 / 1186$ & 93.40 & -1.47 & 1.04 \\
\hline A $88 / 1187$ & 93.50 & -1.65 & 1.30 \\
\hline A $88 / 1188$ & 93.60 & -1.96 & 0.81 \\
\hline A $88 / 1189$ & 93.70 & -1.75 & 0.95 \\
\hline A $88 / 1190$ & 93.80 & -1.48 & 1.35 \\
\hline A $88 / 1191$ & 93.90 & -1.39 & 1.14 \\
\hline A $88 / 1192$ & 94.00 & -1.41 & 1.18 \\
\hline A $88 / 1193$ & 94.10 & -1.63 & 1.16 \\
\hline A $88 / 1194$ & 94.18 & -1.73 & 1.35 \\
\hline A $88 / 1195$ & 94.30 & -1.93 & 1.35 \\
\hline A $88 / 1196$ & 94.40 & -1.88 & 1.50 \\
\hline A $88 / 1197$ & 94.50 & -2.03 & 1.41 \\
\hline A $88 / 1198$ & 94.60 & -1.91 & 1.60 \\
\hline A $88 / 1199$ & 94.70 & -1.60 & 1.60 \\
\hline A $88 / 1200$ & 94.80 & -1.55 & 1.09 \\
\hline A $88 / 1201$ & 94.90 & -1.73 & 0.93 \\
\hline A $88 / 1202$ & 95.00 & -1.53 & 1.08 \\
\hline A $88 / 1203$ & 95.10 & -1.46 & 1.44 \\
\hline A $88 / 1204$ & 95.20 & - & 0.94 \\
\hline A $88 / 1205$ & 95.30 & -1 & 1.24 \\
\hline A $88 / 1206$ & 95.41 & -1.33 & 1.63 \\
\hline A $88 / 1207$ & 95.50 & -1.51 & 1.58 \\
\hline A $88 / 1208$ & 95.60 & -1.52 & 1.38 \\
\hline A $88 / 1209$ & 95.68 & -1.52 & 1.52 \\
\hline A $88 / 1210$ & 95.80 & -1.54 & 1.65 \\
\hline A $88 / 1211$ & 95.90 & -1.67 & 1.88 \\
\hline A $88 / 1212$ & 96.00 & -1.71 & 1.84 \\
\hline A $88 / 1213$ & 96.10 & -1.66 & 1.84 \\
\hline
\end{tabular}


Table 2 (continued).

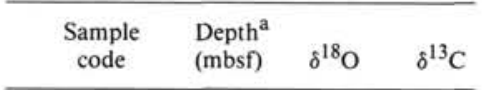

Hole 677A (Cont.)

\begin{tabular}{|c|c|c|c|c|c|c|c|}
\hline A $88 / 1214$ & 96.20 & -1.64 & 1.95 & A $88 / 1794$ & 103.68 & -1.69 & 1.48 \\
\hline A $88 / 1215$ & 96.30 & -1.56 & 1.75 & A $88 / 1795$ & 103.80 & -2.03 & 1.52 \\
\hline A $88 / 1260$ & 96.30 & -1.40 & 1.29 & A $88 / 1796$ & 103.90 & -1.66 & 1.49 \\
\hline A $88 / 1216$ & 96.40 & -1.49 & 1.85 & A $88 / 1797$ & 104.00 & -1.87 & 1.38 \\
\hline A $88 / 1217$ & 96.50 & -1.54 & 1.80 & A $88 / 1798$ & 104.10 & -1.65 & 1.63 \\
\hline A $88 / 1218$ & 96.60 & -1.47 & 1.18 & A $88 / 1799$ & 104.20 & -1.96 & 1.17 \\
\hline A $88 / 1219$ & 96.70 & -0.95 & 1.88 & A $88 / 1800$ & 104.30 & -1.75 & 1.59 \\
\hline A $88 / 1220$ & 96.80 & -1.54 & 1.69 & A $88 / 1806$ & 104.40 & -1.97 & 1.71 \\
\hline A $88 / 1221$ & 96.91 & -1.47 & 1.74 & A $88 / 1808$ & 104.60 & -1.36 & 1.82 \\
\hline A $88 / 1222$ & 97.00 & -1.71 & 1.56 & A $88 / 1809$ & 104.70 & -1.94 & 1.38 \\
\hline A $88 / 1223$ & 97.10 & -1.71 & 1.66 & A $88 / 1810$ & 104.80 & -1.67 & 1.46 \\
\hline A $88 / 1224$ & 97.18 & -1.86 & 1.79 & A $88 / 1821$ & 104.87 & -1.75 & 1.81 \\
\hline A $88 / 1225$ & 97.30 & -1.72 & 1.87 & A $88 / 1813$ & 105.00 & -1.82 & 1.56 \\
\hline A $88 / 1226$ & 97.40 & -1.60 & 1.84 & A $88 / 1814$ & 105.10 & -1.74 & 1.87 \\
\hline A $88 / 1227$ & 97.50 & -1.73 & 1.84 & A $88 / 1815$ & 105.18 & -1.75 & 1.63 \\
\hline A $88 / 1228$ & 97.60 & -1.95 & 1.68 & A $88 / 1816$ & 105.30 & -1.79 & 1.17 \\
\hline A $88 / 1229$ & 97.70 & -1.69 & 1.92 & A $88 / 1817$ & 105.40 & -1.74 & 1.46 \\
\hline A $88 / 1230$ & 97.80 & -1.66 & 1.82 & A $88 / 1818$ & 105.50 & -1.68 & 1.44 \\
\hline A $88 / 1231$ & 97.90 & -1.57 & 1.47 & A $88 / 1819$ & 105.60 & -1.46 & 1.43 \\
\hline A $88 / 1232$ & 98.00 & -1.55 & 1.48 & A $88 / 1820$ & 105.70 & -1.49 & 0.99 \\
\hline A $88 / 1233$ & 98.10 & -1.53 & 1.59 & A $88 / 1822$ & 105.80 & -1.17 & 0.95 \\
\hline A $88 / 1234$ & 98.20 & -1.26 & 1.29 & A $88 / 1823$ & 105.90 & -0.98 & 0.90 \\
\hline A $88 / 1235$ & 98.30 & -1.17 & 1.36 & A $88 / 1824$ & 106.00 & -1.14 & 1.03 \\
\hline A $88 / 1237$ & 98.41 & -1.34 & 1.07 & A $88 / 1825$ & 106.10 & -1.48 & 1.02 \\
\hline A $88 / 1236$ & 98.50 & -1.25 & 1.05 & A $88 / 1826$ & 106.20 & -1.45 & 1.14 \\
\hline A $88 / 1238$ & 98.61 & -1.28 & 1.05 & A $88 / 1828$ & 106.37 & -1.57 & 1.27 \\
\hline A $88 / 1239$ & 98.68 & -1.34 & 0.75 & A $88 / 1829$ & 106.50 & -1.82 & 1.37 \\
\hline A $88 / 1240$ & 98.80 & -1.28 & 0.88 & A $88 / 1830$ & 106.60 & -1.68 & 1.59 \\
\hline A $88 / 1241$ & 98.90 & -1.48 & 0.83 & A $88 / 1831$ & 106.68 & -1.83 & 1.73 \\
\hline A $88 / 1242$ & 99.00 & -1.33 & 0.97 & A $88 / 1832$ & 106.79 & -1.47 & 1.71 \\
\hline A $88 / 1243$ & 99.10 & -1.34 & 1.01 & A $88 / 1833$ & 107.20 & -1.71 & 1.76 \\
\hline A $88 / 1244$ & 99.20 & -1.57 & 1.06 & A $88 / 1834$ & 107.30 & -1.87 & 1.54 \\
\hline A $88 / 1245$ & 99.30 & -1.47 & 0.99 & A $88 / 1835$ & 107.40 & -1.84 & 1.38 \\
\hline A $88 / 1246$ & 99.40 & -1.53 & 1.06 & A $88 / 1836$ & 107.50 & -1.74 & 1.38 \\
\hline A $88 / 1247$ & 99.50 & -1.51 & 1.00 & A $88 / 1837$ & 107.60 & -1.62 & 1.35 \\
\hline A $88 / 1248$ & 99.60 & -1.65 & 1.52 & A $88 / 1838$ & 107.70 & -1.46 & 1.33 \\
\hline A $88 / 1249$ & 99.70 & -1.74 & 1.61 & A $88 / 1839$ & 107.80 & -1.46 & 1.37 \\
\hline A $88 / 1250$ & 99.80 & -1.73 & 1.46 & A $88 / 1840$ & 107.87 & -1.55 & 1.16 \\
\hline A $88 / 1251$ & 99.91 & -1.65 & 1.49 & A $88 / 1841$ & 108.0 & -1.42 & 1.11 \\
\hline A $88 / 1252$ & 100.00 & -1.85 & 1.70 & A $88 / 1842$ & 108.10 & -1.44 & 1.24 \\
\hline A $88 / 1253$ & 100.11 & -1.84 & 1.70 & A $88 / 1843$ & 108.18 & -1.43 & 1.41 \\
\hline A $88 / 1254$ & 100.18 & -1.84 & 1.60 & A $88 / 1844$ & 108.30 & -1.43 & 1.30 \\
\hline A $88 / 1255$ & 100.30 & -1.83 & 1.52 & A $88 / 1845$ & 108.40 & -1.54 & 1.41 \\
\hline A $88 / 1256$ & 100.40 & -1.78 & 1.29 & A $88 / 1846$ & 108.50 & -1.51 & 1.33 \\
\hline A $88 / 1257$ & 100.50 & -1.63 & 1.63 & A $88 / 1847$ & 108.60 & -1.84 & 1.83 \\
\hline A $88 / 1258$ & 100.60 & -1.60 & 1.46 & A $88 / 1848$ & 108.70 & -1.81 & 1.79 \\
\hline A $88 / 1259$ & 100.70 & -1.92 & 1.50 & A $88 / 1849$ & 108.80 & -1.92 & 1.70 \\
\hline A $88 / 1629$ & 100.90 & -1.39 & 1.15 & A $88 / 1850$ & 108.90 & -2.17 & 1.67 \\
\hline A $88 / 1758$ & 101.10 & -1.40 & 0.99 & A $88 / 2048$ & 112.50 & -1.96 & 1.86 \\
\hline A $88 / 1759$ & 101.20 & -1.15 & 0.93 & A $88 / 2050$ & 112.70 & -1.92 & 1.80 \\
\hline A $88 / 1767$ & 101.20 & -1.58 & 1.44 & A $88 / 2051$ & 112.80 & -1.80 & 1.80 \\
\hline A $88 / 1760$ & 101.30 & -1.28 & 0.85 & A $88 / 2052$ & 112.92 & -1.89 & 1.52 \\
\hline A $88 / 1768$ & 101.30 & -1.62 & 1.47 & A $88 / 2053$ & 113.00 & -2.11 & 1.59 \\
\hline A $88 / 1769$ & 101.40 & -1.45 & 1.29 & A $88 / 2055$ & 113.17 & -2.02 & 1.57 \\
\hline A $88 / 1772$ & 101.50 & -1.29 & 1.04 & A $88 / 2056$ & 113.30 & -1.87 & 1.50 \\
\hline A $88 / 1773$ & 101.60 & -1.38 & 0.82 & A $88 / 2057$ & 113.40 & -1.96 & 1.36 \\
\hline A $88 / 1774$ & 101.70 & -1.14 & 0.92 & A $88 / 2058$ & 113.50 & -1.66 & 1.50 \\
\hline A $88 / 1775$ & 101.80 & -1.41 & 0.72 & A $88 / 2059$ & 113.60 & -1.83 & 1.31 \\
\hline A $88 / 1776$ & 101.91 & -1.24 & 0.76 & A $88 / 2061$ & 113.80 & -1.50 & 1.57 \\
\hline A $88 / 1777$ & 102.00 & -1.32 & 0.85 & A $88 / 2062$ & 114.00 & -1.95 & 1.61 \\
\hline A $88 / 1778$ & 102.10 & -1.29 & 0.96 & A $88 / 2063$ & 114.10 & -1.81 & 1.84 \\
\hline A $88 / 1779$ & 102.18 & -1.08 & 0.74 & A $88 / 2066$ & 114.30 & -1.70 & 1.65 \\
\hline A $88 / 1780$ & 102.30 & -1.48 & 0.51 & A $88 / 2067$ & 114.42 & -1.96 & 1.58 \\
\hline A $88 / 1781$ & 102.40 & -1.34 & 0.90 & A $88 / 2068$ & 114.50 & -1.96 & 1.45 \\
\hline A $88 / 1782$ & 102.50 & -1.39 & 0.99 & A $88 / 2069$ & 114.60 & -1.85 & 1.68 \\
\hline A $88 / 1783$ & 102.60 & -1.19 & 1.07 & A $88 / 2070$ & 114.68 & -1.62 & 1.68 \\
\hline A $88 / 1784$ & 102.70 & -0.89 & 1.28 & A $88 / 2071$ & 114.80 & -1.65 & 1.07 \\
\hline A $88 / 1785$ & 102.80 & -1.36 & 1.44 & A $88 / 2072$ & 114.90 & -1.83 & 1.52 \\
\hline A $88 / 1786$ & 102.90 & -1.38 & 1.24 & A $88 / 2073$ & 115.10 & -1.83 & 1.45 \\
\hline A $88 / 1787$ & 103.00 & -1.53 & 1.38 & A $88 / 2074$ & 115.21 & -1.71 & 1.21 \\
\hline A $88 / 1788$ & 103.10 & -1.56 & 1.30 & A $88 / 2075$ & 115.40 & -1.61 & 1.34 \\
\hline A $88 / 1789$ & 103.20 & -1.98 & 1.27 & A $88 / 2077$ & 115.50 & -1.20 & 1.03 \\
\hline A $88 / 1790$ & 103.30 & -1.82 & 1.69 & A $88 / 2078$ & 115.60 & -1.70 & 1.29 \\
\hline A $88 / 1791$ & 103.40 & -1.87 & 1.71 & A $88 / 2079$ & 115.70 & -1.72 & 1.42 \\
\hline A $88 / 1792$ & 103.50 & -1.87 & 1.64 & A $88 / 2080$ & 115.80 & -1.68 & 1.31 \\
\hline A $88 / 1793$ & 103.60 & -1.65 & 1.48 & A $88 / 2081$ & 116.00 & -1.72 & 1.40 \\
\hline
\end{tabular}

Table 2 (continued).

\begin{tabular}{cccc}
\hline $\begin{array}{c}\text { Sample } \\
\text { code }\end{array}$ & $\begin{array}{c}\text { Depth } \\
\text { (mbsf) }\end{array}$ & $\delta^{18} \mathrm{O}$ & $\delta^{13} \mathrm{C}$ \\
\hline
\end{tabular}

Hole 677A (Cont.)

\section{8}

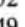

.38

.63
.17

1.17
1.59

1.71

.82

1.38

1.86

.56
.87

1.87
1.63

1.17

.46

1.44

.99

0.95

0.90

.03

.02

1.27

.37

1.59

.73
.71

.76

1.54

.38
.38

1.35

.33

.37

.16
.11

1.24

.41

.41

.33

1.79

.70

.67

.86
.80

1.80

.52

.59

1.57
1.50

36

1.50

1.31
1.57

1.61

1.84
1.65

1.58

1.45

1.68
1.68

1.07

1.52

1.45

1.21
1.34
1.03

1.03

1.29

1.31
1.40
Table 2 (continued).

\begin{tabular}{|c|c|c|c|}
\hline $\begin{array}{l}\text { Sample } \\
\text { code }\end{array}$ & $\begin{array}{l}\text { Depth }{ }^{\mathrm{a}} \\
\text { (mbsf) }\end{array}$ & $\delta^{18} \mathrm{O}$ & $\delta^{13} \mathrm{C}$ \\
\hline \multicolumn{4}{|c|}{ Hole 677B (Cont.) } \\
\hline A $88 / 2084$ & 116.20 & -1.78 & 1.73 \\
\hline A $88 / 2085$ & 116.40 & -1.84 & 1.63 \\
\hline A $88 / 2086$ & 116.50 & -1.89 & 1.66 \\
\hline A $88 / 2087$ & 116.55 & -1.61 & 1.12 \\
\hline A $88 / 2088$ & 116.71 & -2.10 & 1.69 \\
\hline A $88 / 2089$ & 116.80 & -2.19 & 1.53 \\
\hline A $88 / 2091$ & 116.90 & -1.88 & 1.56 \\
\hline A $88 / 2092$ & 117.00 & -1.83 & 1.64 \\
\hline A $88 / 2093$ & 117.10 & -1.95 & 1.57 \\
\hline A $88 / 2094$ & 117.20 & -1.76 & 1.52 \\
\hline A $88 / 2095$ & 117.30 & -1.90 & 1.40 \\
\hline A $88 / 2097$ & 117.50 & -1.72 & 1.39 \\
\hline A $88 / 2098$ & 117.61 & -1.75 & 1.43 \\
\hline A $88 / 2099$ & 117.68 & -1.60 & 1.49 \\
\hline A $88 / 2100$ & 117.80 & -1.46 & 1.71 \\
\hline A $88 / 2101$ & 117.90 & -1.78 & 1.55 \\
\hline A $88 / 2102$ & 118.00 & -1.78 & 1.61 \\
\hline A $88 / 2103$ & 118.10 & -1.86 & 1.51 \\
\hline A $88 / 2104$ & 118.20 & -1.74 & 1.63 \\
\hline A $88 / 2105$ & 118.30 & -1.83 & 1.64 \\
\hline A $88 / 2106$ & 118.40 & -1.84 & 1.65 \\
\hline A $88 / 2107$ & 118.50 & -1.99 & 1.63 \\
\hline A $88 / 2108$ & 118.60 & -1.75 & 1.68 \\
\hline A $88 / 2109$ & 118.70 & -1.91 & 1.70 \\
\hline A $88 / 2110$ & 118.80 & -1.88 & 1.67 \\
\hline A $88 / 2131$ & 118.92 & -2.02 & 1.59 \\
\hline A $88 / 2132$ & 119.00 & -1.77 & 1.59 \\
\hline A $88 / 2133$ & 119.10 & -1.85 & 1.59 \\
\hline A $88 / 2134$ & 119.18 & -1.78 & 1.61 \\
\hline A $88 / 2135$ & 119.30 & -1.74 & 1.55 \\
\hline
\end{tabular}

Hole 677B

$\begin{array}{llll}\text { A 88/721 } & 5.48 & -1.34 & 0.75 \\ \text { A 88/722 } & 5.60 & -0.51 & 0.89 \\ \text { A 88/723 } & 5.70 & -0.53 & 0.86 \\ \text { A 88/724 } & 5.80 & -0.57 & 0.91 \\ \text { A 88/725 } & 5.90 & -0.57 & 0.88 \\ \text { A 88/726 } & 6.00 & -0.56 & 1.09 \\ \text { A 88/727 } & 6.10 & -0.65 & 0.88 \\ \text { A 88/728 } & 6.20 & -0.78 & 0.89 \\ \text { A 88/729 } & 6.30 & -0.95 & 0.81 \\ \text { A 88/730 } & 6.40 & -0.95 & 1.04 \\ \text { A 88/771 } & 6.50 & -0.57 & 0.96 \\ \text { A 88/772 } & 6.60 & -0.87 & 0.61 \\ \text { A 88/773 } & 6.70 & -0.60 & 1.20 \\ \text { A 88/774 } & 6.80 & -0.71 & 0.85 \\ \text { A 88/775 } & 6.90 & -0.60 & 1.12 \\ \text { A 88/776 } & 6.98 & -0.69 & 0.94 \\ \text { A 88/777 } & 7.10 & -0.79 & 1.05 \\ \text { A 88/778 } & 7.20 & -0.75 & 0.73 \\ \text { A 88/779 } & 7.30 & -1.14 & 0.94 \\ \text { A 88/773 } & 14.60 & -0.74 & 1.51 \\ \text { A 88/774 } & 14.70 & -1.21 & 1.45 \\ \text { A 88/775 } & 14.80 & -0.97 & 1.48 \\ \text { A 88/776 } & 14.90 & -0.62 & 1.22 \\ \text { A 88/777 } & 15.00 & -1.31 & 1.43 \\ \text { A 88/778 } & 15.10 & -1.34 & 1.39 \\ \text { A 88/779 } & 15.20 & -1.56 & 1.64 \\ \text { A 88/780 } & 15.30 & -1.42 & 1.30 \\ \text { A 88/821 } & 15.40 & -1.46 & 1.57 \\ \text { A 88/822 } & 15.50 & -1.55 & 1.29 \\ \text { A 88/823 } & 15.60 & -1.59 & 1.60 \\ \text { A 88/824 } & 15.70 & -1.69 & 1.66 \\ \text { A 88/825 } & 15.78 & -1.94 & 1.74 \\ \text { A 88/826 } & 15.90 & -2.02 & 1.94 \\ \text { A 88/827 } & 16.00 & -2.20 & 1.91 \\ \text { A 88/828 } & 16.08 & -2.31 & 1.57 \\ \text { A 88/2441 } & 20.60 & -1.45 & 1.18 \\ \text { A 88/2442 } & 20.70 & -1.42 & 1.44 \\ \text { A 88/2443 } & 20.79 & -1.43 & 1.19 \\ \text { A 88/2444 } & 20.90 & -1.79 & 1.25 \\ \text { A 88/2446 } & 21.10 & -1.72 & 1.58 \\ \text { A 88/2447 } & 21.20 & -1.82 & 1.44 \\ \text { A 88/2448 } & 21.30 & -1.94 & 1.37 \\ \text { A 88/2449 } & 21.40 & -1.92 & 1.32 \\ \text { 8 } & & & \end{array}$


Table 2 (continued).

\begin{tabular}{|c|c|c|c|}
\hline $\begin{array}{l}\text { Sample } \\
\text { code }\end{array}$ & $\begin{array}{l}\text { Depth }^{a} \\
\text { (mbsf) }\end{array}$ & $\delta^{18} \mathrm{O}$ & $\delta^{13} \mathrm{C}$ \\
\hline \multicolumn{4}{|c|}{ Hole 677B (Cont.) } \\
\hline A $88 / 2450$ & 21.50 & -1.65 & 1.44 \\
\hline A $88 / 2451$ & 21.60 & -1.70 & 1.62 \\
\hline A $88 / 2453$ & 21.80 & -1.93 & 1.50 \\
\hline A $88 / 2454$ & 21.90 & -1.89 & 1.18 \\
\hline A $88 / 2455$ & 22.00 & -1.44 & 1.03 \\
\hline A $88 / 2456$ & 22.10 & -0.98 & 0.77 \\
\hline A $88 / 2381$ & 24.60 & -1.71 & 1.39 \\
\hline A $88 / 2382$ & 24.70 & -2.04 & 1.45 \\
\hline A $88 / 2383$ & 24.80 & -1.94 & 1.74 \\
\hline A $88 / 2384$ & 24.90 & -1.83 & 1.56 \\
\hline A $88 / 2385$ & 25.00 & -1.63 & 1.71 \\
\hline A $88 / 2386$ & 25.10 & -1.60 & 1.54 \\
\hline A $88 / 2387$ & 25.20 & -1.37 & 1.28 \\
\hline A $88 / 2388$ & 25.30 & -1.18 & 1.18 \\
\hline A $88 / 2389$ & 25.40 & -1.51 & 1.30 \\
\hline A $88 / 2390$ & 25.50 & -1.59 & 1.22 \\
\hline A $88 / 2391$ & 25.60 & -1.44 & 1.14 \\
\hline A $88 / 2392$ & 25.70 & -0.73 & 0.95 \\
\hline A $88 / 2393$ & 25.80 & -0.73 & 1.07 \\
\hline A $88 / 2394$ & 25.90 & -0.74 & 1.05 \\
\hline A $88 / 2395$ & 26.00 & -0.98 & 1.27 \\
\hline A $87 / 2162$ & 34.10 & -1.22 & 1.12 \\
\hline A $87 / 2163$ & 34.20 & -0.97 & 1.02 \\
\hline A $87 / 2164$ & 34.30 & -0.63 & 1.34 \\
\hline A $87 / 2165$ & 34.40 & -0.87 & 1.35 \\
\hline A $87 / 2166$ & 34.50 & -1.38 & 1.55 \\
\hline A $87 / 2167$ & 34.60 & -1.38 & 1.62 \\
\hline A $87 / 2168$ & 34.70 & -1.71 & 1.64 \\
\hline A $87 / 2169$ & 34.80 & -1.37 & 1.63 \\
\hline A $87 / 2170$ & 34.90 & -1.80 & 1.50 \\
\hline A $87 / 2171$ & 35.00 & -1.25 & 1.55 \\
\hline A $87 / 2172$ & 35.10 & -1.62 & 1.57 \\
\hline A $87 / 2173$ & 35.20 & -1.43 & 1.55 \\
\hline A $87 / 2174$ & 35.30 & -1.36 & 1.64 \\
\hline A $87 / 2175$ & 35.40 & -1.34 & 1.47 \\
\hline A $87 / 2176$ & 35.50 & -1.56 & 1.64 \\
\hline A $88 / 2396$ & 43.60 & -1.68 & 1.35 \\
\hline A $88 / 2542$ & 43.70 & -1.85 & 1.48 \\
\hline A $88 / 2398$ & 43.80 & -1.74 & 1.29 \\
\hline A $88 / 2399$ & 43.90 & -1.72 & 1.36 \\
\hline A $88 / 2400$ & 44.00 & -1.78 & 0.99 \\
\hline A $88 / 2401$ & 44.10 & -1.11 & 1.36 \\
\hline A $88 / 2402$ & 44.20 & -1.13 & 1.30 \\
\hline A $88 / 2403$ & 44.30 & -1.03 & 1.15 \\
\hline A $88 / 2404$ & 44.40 & -1.02 & 1.09 \\
\hline A $88 / 2405$ & 44.50 & -1.17 & 1.27 \\
\hline A $88 / 2406$ & 44.60 & -1.29 & 1.11 \\
\hline A $88 / 2407$ & 44.70 & -1.41 & 1.18 \\
\hline A $88 / 2408$ & 44.80 & -1.34 & 1.18 \\
\hline A $88 / 2409$ & 44.90 & -1.49 & 1.13 \\
\hline A $88 / 2410$ & 45.00 & -1.41 & 1.23 \\
\hline A $88 / 2457$ & 47.90 & -1.11 & 1.07 \\
\hline A $88 / 2458$ & 48.00 & -1.12 & 1.24 \\
\hline A $88 / 2459$ & 48.10 & -1.23 & 1.26 \\
\hline A $88 / 2460$ & 48.20 & -1.35 & 1.52 \\
\hline A $88 / 2544$ & 48.30 & -1.81 & 1.39 \\
\hline A $88 / 2545$ & 48.40 & -1.77 & 1.53 \\
\hline A $88 / 2546$ & 48.50 & -1.68 & 1.24 \\
\hline A $88 / 2547$ & 48.61 & -1.62 & 1.18 \\
\hline A $88 / 2548$ & 48.70 & -1.79 & 1.21 \\
\hline A $88 / 2549$ & 48.80 & -1.23 & 0.91 \\
\hline A $88 / 2550$ & 48.90 & -1.33 & 0.88 \\
\hline A $88 / 2551$ & 49.00 & -1.05 & 0.92 \\
\hline A $88 / 2552$ & 49.10 & -1.41 & 0.83 \\
\hline A $88 / 2553$ & 49.20 & -1.49 & 1.03 \\
\hline A $88 / 2554$ & 49.30 & -1.35 & 1.01 \\
\hline A $88 / 2555$ & 49.40 & -1.35 & 1.04 \\
\hline A $88 / 2411$ & 53.11 & -1.57 & 1.18 \\
\hline A $88 / 829$ & 53.11 & -1.34 & 0.69 \\
\hline A $88 / 830$ & 53.20 & -1.17 & 1.01 \\
\hline A $88 / 2412$ & 53.20 & -1.48 & 1.04 \\
\hline A $88 / 2413$ & 53.30 & -1.30 & 1.12 \\
\hline
\end{tabular}

Table 2 (continued).

\begin{tabular}{|c|c|c|c|}
\hline $\begin{array}{l}\text { Sample } \\
\text { code }\end{array}$ & $\begin{array}{l}\text { Depth } \\
\text { (mbsf) }\end{array}$ & $\delta^{18} \mathrm{O}$ & $\delta^{13} \mathrm{C}$ \\
\hline \multicolumn{4}{|c|}{ Hole 677B (Cont.) } \\
\hline A $88 / 2414$ & 53.40 & -1.33 & 0.80 \\
\hline A $88 / 2415$ & 53.50 & -1.24 & 1.00 \\
\hline A $88 / 2416$ & 53.60 & -1.33 & 0.86 \\
\hline A $88 / 2417$ & 53.70 & -1.40 & 0.95 \\
\hline A $88 / 2418$ & 53.80 & -1.51 & 0.81 \\
\hline A $88 / 2419$ & 53.90 & -1.40 & 0.88 \\
\hline A $88 / 2420$ & 54.00 & -1.56 & 1.14 \\
\hline A $88 / 2421$ & 54.10 & -1.37 & 1.18 \\
\hline A $88 / 2422$ & 54.20 & -1.63 & 1.21 \\
\hline A $88 / 2423$ & 54.30 & -1.76 & 1.35 \\
\hline A $88 / 2424$ & 54.40 & -1.65 & 1.31 \\
\hline A $88 / 2425$ & 54.50 & -1.79 & 1.44 \\
\hline A $88 / 2426$ & 62.61 & -1.74 & 1.52 \\
\hline A $88 / 2427$ & 62.70 & -1.90 & 1.39 \\
\hline A $88 / 2428$ & 62.80 & -1.48 & 1.43 \\
\hline A $88 / 2429$ & 62.90 & -1.54 & 1.41 \\
\hline A $88 / 2430$ & 63.00 & -1.57 & 1.55 \\
\hline A $88 / 2431$ & 63.10 & -1.54 & 1.39 \\
\hline A $88 / 2432$ & 63.20 & -1.46 & 1.27 \\
\hline A $88 / 2433$ & 63.30 & -1.16 & 1.20 \\
\hline A $88 / 2434$ & 63.40 & -1.27 & 1.01 \\
\hline A $88 / 2435$ & 63.50 & -0.89 & 0.85 \\
\hline A $88 / 2436$ & 63.58 & -0.80 & 0.88 \\
\hline A $88 / 2543$ & 63.70 & -0.96 & 0.84 \\
\hline A $88 / 2438$ & 63.80 & -0.88 & 0.68 \\
\hline A $88 / 2439$ & 63.90 & -0.71 & 0.71 \\
\hline A $88 / 2440$ & 64.00 & -1.09 & 0.79 \\
\hline A $88 / 2556$ & 72.11 & -1.45 & 1.24 \\
\hline A $88 / 2557$ & 72.20 & -1.55 & 1.19 \\
\hline A $88 / 2558$ & 72.30 & -1.51 & 0.82 \\
\hline A $88 / 2559$ & 72.40 & -1.59 & 0.82 \\
\hline A $88 / 2560$ & 72.50 & -1.48 & 1.07 \\
\hline A $88 / 2561$ & 72.60 & -0.96 & 0.83 \\
\hline A $88 / 2563$ & 72.80 & -1.40 & 0.81 \\
\hline A $88 / 2564$ & 72.90 & -1.33 & 0.79 \\
\hline A $88 / 2565$ & 73.00 & -1.05 & 1.10 \\
\hline A $88 / 2566$ & 81.60 & -1.48 & 1.03 \\
\hline A $88 / 2567$ & 81.70 & -1.52 & 1.19 \\
\hline A $88 / 2568$ & 81.80 & -1.53 & 0.92 \\
\hline A $88 / 2570$ & 82.00 & -1.37 & 0.88 \\
\hline A $88 / 2591$ & 82.10 & -1.49 & 0.81 \\
\hline A $88 / 2593$ & 82.30 & -1.37 & 0.82 \\
\hline A $88 / 2594$ & 82.40 & -1.32 & 1.07 \\
\hline A $88 / 2595$ & 82.50 & -1.53 & 0.83 \\
\hline A $88 / 2596$ & 82.60 & -1.48 & 0.79 \\
\hline A $88 / 2597$ & 82.70 & -1.33 & 0.75 \\
\hline A $88 / 2598$ & 82.80 & -1.36 & 0.90 \\
\hline A $88 / 2599$ & 82.90 & -1.01 & 1.22 \\
\hline A $88 / 2600$ & 83.00 & -1.52 & 0.86 \\
\hline A $88 / 2631$ & 91.10 & -1.54 & 0.86 \\
\hline A $88 / 2632$ & 91.20 & -1.68 & 0.87 \\
\hline A $88 / 2633$ & 91.30 & -1.40 & 0.61 \\
\hline A $88 / 2634$ & 91.40 & -1.36 & 0.87 \\
\hline A $88 / 2635$ & 91.50 & -1.61 & 1.14 \\
\hline A $88 / 2636$ & 91.60 & -1.69 & 1.13 \\
\hline A $88 / 2637$ & 91.70 & -1.70 & 1.32 \\
\hline A $88 / 2638$ & 91.80 & -2.05 & 1.44 \\
\hline A $88 / 2639$ & 91.90 & -1.75 & 1.36 \\
\hline A $88 / 2640$ & 92.00 & -1.71 & 1.54 \\
\hline A $88 / 2641$ & 92.10 & -1.65 & 1.39 \\
\hline A $88 / 2642$ & 92.20 & -1.79 & 1.10 \\
\hline A $88 / 2643$ & 92.30 & -1.72 & 1.22 \\
\hline A $88 / 2644$ & 92.40 & -1.69 & 1.06 \\
\hline A $88 / 2645$ & 92.50 & -1.63 & 0.97 \\
\hline A $88 / 2646$ & 92.60 & -1.63 & 1.10 \\
\hline
\end{tabular}

a Depth from barrel sheets (Shipboard Scientific Party, 1988).

b Unreliable measurement or downhole contamination.

${ }^{\mathrm{c}}$ Species from $300-425-\mu \mathrm{m}$ fraction. 
Table 3. Oxygen and carbon isotope data for benthic foraminifers in Holes 677A and 677B.

\begin{tabular}{lllll}
\hline $\begin{array}{c}\text { Sample } \\
\text { code }\end{array}$ & $\begin{array}{c}\text { Depth } \\
\text { (mbsf) }\end{array}$ & Species $^{\mathrm{b}}$ & $\delta^{18} \mathrm{O}$ & $\delta^{13} \mathrm{C}$ \\
\hline Hole 677A & & & &
\end{tabular}

\begin{tabular}{|c|c|c|c|c|}
\hline A $87 / 1614$ & 0.40 & 1 & 3.93 & -0.95 \\
\hline A $87 / 1615$ & 0.50 & 1 & 4.27 & -1.10 \\
\hline A $87 / 1616$ & 0.60 & 1 & 4.58 & -1.18 \\
\hline A $87 / 1617$ & 0.68 & 1 & 4.22 & -1.22 \\
\hline A $87 / 1618$ & 0.80 & 1 & 4.80 & -1.40 \\
\hline A $87 / 1619$ & 0.90 & 1 & 4.99 & -1.29 \\
\hline A $87 / 1620$ & 0.98 & 1 & 5.07 & -1.31 \\
\hline A $88 / 543$ & 1.10 & 1 & 5.23 & -1.09 \\
\hline A $88 / 846$ & 1.10 & 1 & 5.07 & -1.30 \\
\hline A $87 / 1621$ & 1.20 & 1 & 5.13 & -1.27 \\
\hline A $87 / 1622$ & 1.30 & 1 & 4.94 & -1.24 \\
\hline A $87 / 1623$ & 1.40 & 1 & 5,19 & -1.11 \\
\hline A $88 / 544$ & 1.50 & 1 & 4.94 & -1.13 \\
\hline A $88 / 545$ & 1.60 & 1 & 4.94 & -1.14 \\
\hline A $88 / 546$ & 1.70 & 1 & 4.77 & -1.22 \\
\hline A $88 / 547$ & 1.80 & 1 & 4.78 & -1.11 \\
\hline A $88 / 548$ & 1.90 & 1 & 4.77 & -1.10 \\
\hline A $88 / 549$ & 2.00 & 1 & 4.81 & -1.11 \\
\hline A $88 / 550$ & 2.10 & 1 & 4.69 & -1.09 \\
\hline A $88 / 551$ & 2.20 & 1 & 4.64 & -1.12 \\
\hline A $88 / 552$ & 2.30 & 1 & 4.56 & -1.18 \\
\hline A $88 / 553$ & 2.40 & 1 & 4.66 & -1.10 \\
\hline A $88 / 554$ & 2.47 & 1 & 4.71 & -1.02 \\
\hline A $88 / 555$ & 2.58 & 1 & 4.60 & -1.15 \\
\hline A $88 / 556$ & 2.70 & 1 & 4.59 & -1.07 \\
\hline A $88 / 557$ & 2.80 & 1 & 4.58 & -1.28 \\
\hline A $88 / 576$ & 2.90 & 1,2 & 4.55 & -1.36 \\
\hline A $88 / 577$ & 2.90 & 3 & 3.71 & -0.39 \\
\hline A $88 / 558$ & 3.00 & 1 & 4.58 & -1.04 \\
\hline A $88 / 578$ & 3.10 & 1 & 4.62 & -1.08 \\
\hline A $88 / 579$ & 3.20 & 1 & 4.54 & -1.23 \\
\hline A $88 / 559$ & 3.30 & 1 & 4.55 & -1.33 \\
\hline A $88 / 560$ & 3.40 & 1 & 4.67 & -1.27 \\
\hline A $88 / 580$ & 3.50 & 1 & 4.71 & -1.23 \\
\hline A $88 / 621$ & 3.60 & 1 & 4.72 & -1.29 \\
\hline A $88 / 622$ & 3.70 & 1 & 4.74 & -1.25 \\
\hline A $88 / 623$ & 3.80 & 1 & 4.78 & -1.26 \\
\hline A $88 / 624$ & 3.90 & 1 & 4.57 & -1.04 \\
\hline A $88 / 526$ & 3.97 & 2 & 4.70 & -1.37 \\
\hline A $88 / 625$ & 3.97 & 1 & 4.71 & -1.18 \\
\hline A $88 / 527$ & 4.08 & 4 & 4.90 & 1.56 \\
\hline A $88 / 626$ & 4.08 & 1 & 4.43 & -1.04 \\
\hline A $88 / 630$ & 4.20 & 1,2 & 4.21 & -1.01 \\
\hline A $88 / 911$ & 4.20 & 4 & 4.74 & 1.65 \\
\hline A $88 / 528$ & 4.30 & 1 & 4.45 & -1.02 \\
\hline A $88 / 912$ & 4.30 & 5 & 3.57 & -0.56 \\
\hline A $88 / 529$ & 4.60 & 1 & 4.14 & -1.00 \\
\hline A $88 / 913$ & 4.60 & 3,6 & 3.51 & -0.37 \\
\hline A $88 / 530$ & 4.70 & 1 & 4.25 & -1.06 \\
\hline A $88 / 914$ & 4.70 & $5,6,7$ & 3.64 & -0.59 \\
\hline A $88 / 531$ & 4.80 & 1 & 4.43 & -0.90 \\
\hline A $88 / 915$ & 4.80 & 3,5 & 3.64 & -0.15 \\
\hline A $88 / 916$ & 4.80 & 1 & 4.31 & -1.22 \\
\hline A $88 / 532$ & 4.90 & 1 & 4.25 & -0.87 \\
\hline A $88 / 917$ & 4.90 & 1 & 4.15 & -1.03 \\
\hline A $88 / 918$ & 4.90 & 3,5 & 3.52 & -0.25 \\
\hline A $88 / 919$ & 4.90 & 2 & 4.26 & -1.23 \\
\hline A $88 / 920$ & 4.90 & 4 & 4.79 & 1.53 \\
\hline A $88 / 533$ & 4.98 & 1 & 4.09 & -0.90 \\
\hline A $88 / 921$ & 4.98 & 4 & 4.62 & 1.54 \\
\hline A $88 / 534$ & 5.13 & 1 & 4.30 & -1.01 \\
\hline A $88 / 922$ & 5.13 & 1 & 4.38 & -0.97 \\
\hline A $88 / 923$ & 5.13 & 4 & ${ }^{c} 5.24$ & 1.42 \\
\hline A $88 / 535$ & 5.20 & 1 & 3.78 & -1.22 \\
\hline A $88 / 924$ & 5.20 & 2 & 3.89 & -1.57 \\
\hline A $88 / 925$ & 5.20 & 3 & 3.39 & -0.34 \\
\hline A $88 / 536$ & 5.30 & 1 & 4.01 & -1.14 \\
\hline A $88 / 860$ & 5.30 & 1,2 & 4.01 & -1.13 \\
\hline A $88 / 537$ & 5.40 & 1 & 4.15 & -1.04 \\
\hline A $88 / 858$ & 5.40 & 2 & 4.28 & -1.55 \\
\hline A $88 / 859$ & 5.40 & 3 & 3.51 & -0.73 \\
\hline A $88 / 542$ & 5.60 & 1,2 & 3.94 & -1.38 \\
\hline A $88 / 856$ & 5.60 & 3 & 3.42 & -0.17 \\
\hline A $88 / 857$ & 5.60 & 2 & 4.13 & -1.45 \\
\hline A $88 / 538$ & 5.70 & 1 & 4.30 & -1.01 \\
\hline A $88 / 854$ & 5.70 & 3 & 3.10 & -0.25 \\
\hline A $88 / 855$ & 5.70 & 2 & 3.78 & -1.47 \\
\hline A $88 / 539$ & 5.80 & 1,2 & 4.05 & -1.28 \\
\hline A $88 / 853$ & 5.80 & 3,6 & 3.11 & -0.66 \\
\hline
\end{tabular}

Table 3 (continued).

\begin{tabular}{|c|c|c|c|c|}
\hline $\begin{array}{c}\text { Sample } \\
\text { code }\end{array}$ & $\begin{array}{l}\text { Depth }{ }^{a} \\
\text { (mbsf) }\end{array}$ & Species ${ }^{b}$ & $\delta^{18} \mathrm{O}$ & $\delta^{13} \mathrm{C}$ \\
\hline
\end{tabular}
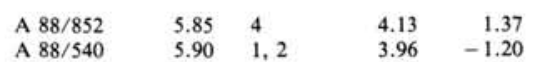

$\begin{array}{lllll}\text { A } 88 / 540 & 5.90 & 1,2 & 3.96 & -1.20 \\ \text { A } 88 / 850 & 5.90 & 3 & 2.66 & -0.18\end{array}$

$\begin{array}{lllll}\text { A } 88 / 851 & 5.90 & 2,8 & 3.64 & -1.38 \\ \text { A } 88 / 541 & 6.00 & 1 & 4.24 & -0.92\end{array}$

$\begin{array}{lllll}\text { A } 88 / 848 & 6.00 & 1,2 & 4.11 & -1.08\end{array}$

$\begin{array}{lllll}\text { A } 88 / 848 & 6.00 & 1,2 & 4.11 & -1.08 \\ \text { A } 88 / 849 & 6.05 & 3 & 2.64 & -0.30\end{array}$

$\begin{array}{lllll}\text { A } 88 / 847 & 6.10 & 1,2 & 3.44 & -1.13\end{array}$

$\begin{array}{lllll}\text { A } 88 / 627 & 6.59 & 1 & 4.68 & -1.51 \\ \text { A } 88 / 628 & 6.68 & 1 & 4.60 & -1.56\end{array}$

$\begin{array}{lllll}\text { A } 88 / 629 & 6.80 & 1 & 4.36 & -1.48\end{array}$

$\begin{array}{lllll}\text { A } 87 / 2030 & 6.92 & 1 & 4.56 & -1.31\end{array}$

$\begin{array}{lllll}\text { A } 88 / 631 & 7.18 & 3 & 2.98 & -0.49 \\ \text { A } 87 / 2029 & 7.29 & 1 & 3.97 & -1.05\end{array}$

$\begin{array}{lllll}\text { A } 87 / 2029 & 7.29 & 1 & 3.97 & -1.05 \\ \text { A } 88 / 632 & 7.49 & 1,2 & 3.96 & -1.19\end{array}$

$\begin{array}{lllll}\text { A } 88 / 633 & 7.59 & 1,2 & 3.70 & -1.53 \\ \text { A } 87 / 2028 & 7.80 & 1 & 3.97 & -1.07\end{array}$

$\begin{array}{lllll}\text { A } 87 / 2028 & 7.90 & 1.2 & 4.01 & -1.47\end{array}$

$\begin{array}{lllll}\text { A } 87 / 2026 & 8.11 & 1 & 4.67 & -1.41\end{array}$

$\begin{array}{lllll}\text { A } 87 / 2025 & 8.25 & 1 & 4.55 & -1.35\end{array}$

$\begin{array}{lllll}\text { A } 87 / 2024 & 8.43 & 1 & 4.69 & -1.25 \\ \text { A } 87 / 2023 & 8.75 & 1 & 4.22 & -1.10\end{array}$

A $87 / 2022 \quad 8.90 \quad 1.2 \quad 3.87 \quad-1.17$

$\begin{array}{lllll}\text { A } 87 / 2019 & 9.05 & 1 & 4.18 & -1.17\end{array}$

$\begin{array}{lllll}\text { A } 87 / 2018 & 9.20 & 1 & 4.71 & -1.38 \\ \text { A } 87 / 2017 & 9.29 & 1 & 4.63 & -1.28\end{array}$

$\begin{array}{lllll}\text { A } 87 / 2017 & 9.29 & 1 & 4.63 & -1.28 \\ \text { A } 87 / 2016 & 9.39 & 1 & 4.92 & -1.34\end{array}$

$\begin{array}{lllrr}\text { A } 87 / 2015 & 9.49 & 1 & 4.92 & -1.34 \\ \text { A } 87 / 2013 & 9.59 & 4 & 5.81 & -1.36 \\ \text { A } 87 / 2014 & 9.59 & 1 & 5.17 & 0.99\end{array}$

$\begin{array}{lllll}\text { A } 87 / 2014 & 9.59 & 1 & 4.69 & -1.63\end{array}$

$\begin{array}{lllll}\text { A } 87 / 2011 & 9.69 & 1 & 4.91 & -1.32\end{array}$

$\begin{array}{lllll}\text { A } 87 / 2010 & 9.78 & 1 & 4.78 & -1.65 \\ \text { A } 87 / 2009 & 9.92 & 1 & 4.74 & -1.63\end{array}$

$\begin{array}{lrlll}\text { A } 87 / 2009 & 9.92 & 1 & 4.74 & -1.63 \\ \text { A } 87 / 2008 & 10.00 & 1 & 4.70 & -1.71\end{array}$

$\begin{array}{lllll}\text { A } 87 / 2007 & 10.10 & 1 & 5.08 & -1.53\end{array}$

$\begin{array}{lllll}\text { A } 87 / 2006 & 10.29 & 1 & 4.64 & -1.39\end{array}$

$\begin{array}{lllll}\text { A } 877 / 2005 & 10.49 & & 4.50 & -1.64 \\ \text { A } 87 / 2004 & 10.59 & 1,2 & 4.07 & -1.72\end{array}$

$\begin{array}{lllll}\text { A } 87 / 2004 & 10.59 & 1,2 & 4.07 & -1.72 \\ \text { A } 87 / 2002 & 10.77 & 1,2 & 3.83 & -1.75\end{array}$

$\begin{array}{lllll}\text { A } 87 / 2003 & 10.77 & 1.2 & 4.10 & -1.52\end{array}$

$\begin{array}{lllll}\text { A } 87 / 2001 & 10.89 & 1 & 4.26 & -1.44 \\ \text { A } 87 / 1997 & 10.99 & 1 & 4.39 & -1.65\end{array}$

$\begin{array}{lllll}\text { A } 87 / 1996 & 11.11 & 1,2 & 4.52 & -1.80\end{array}$

$\begin{array}{lllll}\text { A } 87 / 1995 & 11.17 \quad 1 & 4.50 & -1.51\end{array}$

$\begin{array}{lllll}\text { A } 87 / 1994 & 11.29 & 1 & 4.30 & -1.67\end{array}$

$\begin{array}{lllll}\text { A } 87 / 1993 & 11.40 & 1 & 4.32 & -1.45\end{array}$

$\begin{array}{lllll}\text { A } 87 / 1992 & 11.49 & 1 & 4.12 & -1.57 \\ \text { A } 87 / 1991 & 11.60 & 1 & 4.41 & -1.50\end{array}$

$\begin{array}{lllll}\text { A } 87 / 1970 & 11.68 & 1 & 4.08 & -1.43\end{array}$

$\begin{array}{lllll}\text { A } 87 / 1969 & 11.79 & 1 & 3.81 & -1.57\end{array}$

$\begin{array}{lllll}\text { A } 87 / 1968 & 11.89 & 1 & 4.07 & -1.34\end{array}$

$\begin{array}{lllll}\text { A } 87 / 1966 & 11.99 & 2 & 3.78 & -1.63\end{array}$

$\begin{array}{llllr}\text { A } 87 / 1967 & 11.99 & 3 & 2.96 & -0.57 \\ \text { A } 87 / 1965 & 12.09 & 4 & 4.13 & 1.17\end{array}$

$\begin{array}{llllr}\text { A } 87 / 1965 & 12.09 & 4 & 4.13 & 1.17 \\ \text { A } 87 / 1964 & 12.20 & 2 & 3.25 & -1.42\end{array}$

$\begin{array}{lllll}\text { A } 87 / 1963 & 12.29 & 4 & 4.09 & 1.61\end{array}$

$\begin{array}{lllll}\text { A } 87 / 1961 & 12.39 & 4 & 3.89 & 1.06\end{array}$

$\begin{array}{lllll}\text { A } 87 / 1962 & 12.39 & 1,2 & 3.51 & -1.68\end{array}$

$\begin{array}{lllll}\text { A } 87 / 1960 & 12.49 & 1 & 3.81 & -1.51\end{array}$

$\begin{array}{lllll}\text { A } 87 / 1959 & 12.59 & 1 & 4.42 & -1.46 \\ \text { A } 87 / 1958 & 12.69 & 1 & 4.78 & -1.58\end{array}$

$\begin{array}{lllll}\text { A } 87 / 1958 & 12.69 & 1 & 4.78 & -1.58 \\ \text { A } 87 / 1957 & 12.79 & 1 & 5.27 & -1.32\end{array}$

$\begin{array}{lllll}\text { A } 87 / 1956 & 12.92 & 1 & 5.12 & -1.61\end{array}$

$\begin{array}{lllll}\text { A } 87 / 1955 & 13.01 & 1 & 5.06 & -1.60\end{array}$

$\begin{array}{lllll}\text { A } 87 / 1954 & 13.10 & 1 & 4.68 & -1.75\end{array}$

$\begin{array}{lllll}\text { A } 87 / 1953 & 13.18 & 1 & 4.80 & -1.54 \\ \text { A } 87 / 1952 & 13.30 & 1 & 4.87 & -1.61\end{array}$

$\begin{array}{lllll}\text { A } 87 / 1940 & 13.52 & 1,2 & 4.80 & -1.74\end{array}$

$\begin{array}{lllll}\text { A } 87 / 1939 & 13.60 & 2 & 4.71 & -2.05\end{array}$

$\begin{array}{lllll}\text { A } 87 / 1938 & 13.70 & 1 & 4.53 & -1.34\end{array}$

$\begin{array}{lllll}\text { A } 87 / 1937 & 13.80 & 1,2 & 4.62 & -1.34\end{array}$

$\begin{array}{lllll}\text { A } 87 / 1936 & 13.89 & 1 & 4.64 & -1.13 \\ \text { A } 87 / 1935 & 14.00 & 1 & 4.37 & -1.42\end{array}$

$\begin{array}{lllll}\text { A } 87 / 1934 & 14.10 & 1 & 4.56 & -1.39\end{array}$

$\begin{array}{lllll}\text { A } 87 / 1932 & 14.30 & 1 & 4.12 & -1.30\end{array}$

$\begin{array}{lllll}\text { A } 87 / 1931 & 14.42 & 1 & 4.33 & -1.78 \\ \text { A } 87 / 1911 & 14.50 & 1 & 4.27 & -1.23\end{array}$

$\begin{array}{lllll}\text { A } 87 / 1912 & 14.60 & 1 & 4.27 & -1.23 \\ \text { A } 87 / 1913 & 14.68 & 1 & 3.88 & -0.95\end{array}$

$\begin{array}{lllll}\text { A } 87 / 1913 & 14.68 & 1 & 3.85 & -0.95 \\ \text { A } 87 / 1915 & 14.89 & 1 & 3.71 & -0.82\end{array}$
Table 3 (continued).

\begin{tabular}{ccccc}
\hline $\begin{array}{c}\text { Sample } \\
\text { code }\end{array}$ & $\begin{array}{c}\text { Depth } \\
\text { (mbsf) }\end{array}$ & Species $^{\mathrm{b}}$ & $\delta^{18} \mathrm{O}$ & $\delta^{13} \mathrm{C}$ \\
\hline Hole 677A (Cont.)
\end{tabular}

Hole $677 \mathrm{~A}$ (Cont.)

$\begin{array}{llllr}\text { A } 87 / 1916 & 14.99 & 1 & 3.49 & -0.94 \\ \text { A } 87 / 1918 & 15.20 & 1 & 3.50 & -0.88 \\ \text { A } 87 / 1919 & 15.29 & 1 & 3.39 & -1.04 \\ \text { A } 87 / 1920 & 15.39 & 4 & 4.17 & 1.37 \\ \text { A } 87 / 1880 & 15.49 & 1 & 4.01 & -1.36 \\ \text { A } 87 / 1877 & 15.59 & 1 & 4.08 & -1.04 \\ \text { A } 87 / 1878 & 15.59 & 2 & 4.54 & -1.82 \\ \text { A } 87 / 1879 & 15.59 & 4 & 5.01 & 1.09\end{array}$

$\begin{array}{llllr}\text { A } 87 / 1875 & 15.68 & 2 & 4.55 & -1.75\end{array}$

$\begin{array}{llllr}\text { A } 87 / 1876 & 15.68 & 4 & 5.12 & 1.19 \\ \text { A } 87 / 1873 & 15.78 & 1 & 5.14 & -1.43\end{array}$

$\begin{array}{lllll}\text { A } 87 / 1874 & 15.78 & 2 & 4.83 & -1.85\end{array}$

$\begin{array}{lllll}\text { A } 87 / 1511 & 15.80 & 1 & 4.30 & -1.38\end{array}$

$\begin{array}{lllll}\text { A } 87 / 1512 & 16.00 & 3 & 3.94 & -0.41\end{array}$

$\begin{array}{lllll}\text { A } 87 / 1513 & 16.10 & 3 & 3.98 & -0.52 \\ \text { A } 87 / 1515 & 16.20 & 2 & 4.65 & -1.68\end{array}$

$\begin{array}{lllll}\text { A } 87 / 1516 & 16.30 & 1 & 4.70 & -1.48\end{array}$

$\begin{array}{lllll}\text { A } 87 / 1517 & 16.37 & 1 & 4.63 & -1.34 \\ \text { A } 87 / 1518 & 16.50 & 1 & 4.66 & -1.25\end{array}$

$\begin{array}{lllll}\text { A } 87 / 1518 & 16.50 & 1 & 4.66 & -1.25 \\ \text { A } 87 / 1519 & 16.60 & 3 & 3.87 & -0.64\end{array}$

$\begin{array}{lllll}\text { A } 87 / 1519 & 16.60 & 3 & 3.87 & -0.64 \\ \text { A } 87 / 1520 & 16.60 & 2 & 4.27 & -1.99\end{array}$

$\begin{array}{lllll}\text { A } 87 / 1521 & 16.75 & 1 & 4.27 & -1.99 \\ \text { A } 87 / 1523 & 16.86 & 1 & 4.63 & -1.33\end{array}$

$\begin{array}{lllll}\text { A } 87 / 1523 & 16.86 & 1 & 4.65 & -1.35 \\ \text { A } 87 / 1524 & 16.86 & 2 & 4.51 & -1.75\end{array}$

$\begin{array}{lllll}\text { A } 87 / 1525 & 16.90 & 3 & 4.77 & -1.75 \\ \text { A } 87 / 1527 & 17.00 & 3 & 3.87 & -0.38\end{array}$

$\begin{array}{lllll}\text { A } 87 / 1527 & 17.00 & 3 & 3.87 & -0.38\end{array}$

$\begin{array}{lllll}\text { A } 87 / 1528 & 17.10 & 2 & 4.41 & -1.60\end{array}$

$\begin{array}{lllll}\text { A } 87 / 1529 & 17.20 & 3 & 3.76 & -0.28 \\ \text { A } 87 / 1530 & 17.30 & 3 & 3.63 & -0.45\end{array}$

\begin{tabular}{lllll} 
A $87 / 1532$ & 17.40 & 4 & 4.51 & 1.43 \\
\hline
\end{tabular}

$\begin{array}{lllll}\text { A } 87 / 1533 & 17.40 & 2 & 4.03 & -1.53 \\ \text { A } 87 / 1534 & 17.50 & 1.2 & 4.03 & -1.28\end{array}$

$\begin{array}{lllll}\text { A } 87 / 1534 & 17.50 & 1,2 & 4.03 & -1.28 \\ \text { A } 87 / 1535 & 17.60 & 2 & 3.89 & -1.49\end{array}$

$\begin{array}{lllll}\text { A } 87 / 1536 & 17.70 & 1 & 3.76 & -0.94\end{array}$

$\begin{array}{lllll}\text { A } 87 / 1538 & 17.95 & 1 & 3.89 & -0.73 \\ \text { A } 87 / 1539 & 18.10 & 1,2 & 3.89 & -1.13\end{array}$

$\begin{array}{lllll}\text { A } 87 / 1539 & 18.10 & 1,2 & 3.89 & -1.13 \\ \text { A } 87 / 1540 & 18.18 & 1,2 & 3.76 & -0.92\end{array}$

$\begin{array}{lllll}\text { A } 87 / 1541 & 18.30 & 3 & 3.14 & 0.08\end{array}$

$\begin{array}{lllll}\text { A } 87 / 1542 & 18.35 & 1 & 3.94 & -0.91\end{array}$

$\begin{array}{lllll}\text { A } 87 / 1543 & 18.40 & 2 & 3.95 & c^{-1.31}\end{array}$

$\begin{array}{llllr}\text { A } 87 / 1545 & 18.60 & 1 & 3.33 & { }^{c}-1.25 \\ \text { A } 87 / 1871 & 18.60 & 1,2 & 4.14 & -1.23\end{array}$

$\begin{array}{lllrr}\text { A } 87 / 1872 & 18.60 & 4 & 4.62 & -1.23 \\ \text { A } 87 / 1546 & 18.60 & & 4.23 & -1.06\end{array}$

$\begin{array}{lllll}\text { A } 87 / 1546 & 18.70 & 1 & 4.23 & -1.06\end{array}$

$\begin{array}{lllll}\text { A } 87 / 1547 & 18.80 & 1 & 4.23 & -0.92\end{array}$

$\begin{array}{lllll}\text { A } 87 / 1548 & 18.90 & 1 & 4.33 & -0.86 \\ \text { A } 87 / 1549 & 19.00 & 1 & 4.14 & -1.00\end{array}$

$\begin{array}{lllll}\text { A } 87 / 1550 & 19.10 & 1 & 4.14 & -1.00 \\ \text { A } 87 / 1551 & 4.22 & -0.94\end{array}$

$\begin{array}{lllll}\text { A } 87 / 1551 & 19.20 & 1 & 4.03 & -1.04 \\ \text { A } 87 / 1552 & 19.29 & 2 & 3.82 & -1.52\end{array}$

$\begin{array}{lllll}\text { A } 87 / 1553 & 19.35 & 1 & 4.82 & -1.52 \\ \text { A } 87 / 1554 & 19.35 & 3 & 3.36 & -1.00\end{array}$

$\begin{array}{lllll}\text { A } 87 / 1554 & 19.35 & 3 & 3.36 & -0.37\end{array}$

$\begin{array}{lllll}\text { A } 87 / 1556 & 19.48 & 2 & 4.36 & -0.37 \\ \text { A } 87 / 1555 & 19.55 & 1 & 4.10 & -1.67\end{array}$

$\begin{array}{lllll}\text { A } 87 / 1555 & 19.55 & 1 & 4.07 & -1.11\end{array}$

$\begin{array}{lllll}\text { A } 87 / 1558 & 19.67 & 2 & 4.69 & -1.46\end{array}$

$\begin{array}{lllll}\text { A } 87 / 1559 & 19.79 & 1 & 4.42 & -1.37\end{array}$

$\begin{array}{lllll}\text { A } 87 / 1560 & 19.90 & 1 & 4.31 & -1.52 \\ \text { A } 87 / 1561 & 20.00 & 1 & 4.32 & -1.45\end{array}$

$\begin{array}{lllll}\text { A } 87 / 1562 & 20.10 & 1 & 4.50 & -1.23\end{array}$

$\begin{array}{lllll}\text { A } 87 / 1563 & 20.20 & 1 & 4.63 & -1.05\end{array}$

$\begin{array}{lllll}\text { A } 87 / 1565 & 20.40 & 1 & 4.53 & -1.04 \\ \text { A } 87 / 1566 & 20.50 & 1 & 4.24 & -0.98\end{array}$

$\begin{array}{lllll}\text { A } 87 / 1566 & 20.50 & 1 & 4.24 & -0.98 \\ \text { A } 87 / 1567 & 20.60 & 1 & 3.96 & -1.35\end{array}$

$\begin{array}{lllll}\text { A } 87 / 1567 & 20.60 & 1 & 3.96 & -1.35 \\ \text { A } 87 / 1568 & 20.60 & 3 & 3.42 & -0.16\end{array}$

$\begin{array}{lllll}\text { A } 87 / 1569 & 20.70 & 1 & 3.77 & -1.18\end{array}$

$\begin{array}{lllll}\text { A } 87 / 1570 & 20.80 & 1 & 3.72 & -1.06\end{array}$

$\begin{array}{lllll}\text { A } 87 / 1578 & 20.87 & 1 & 3.58 & -1.38 \\ \text { A } 87 / 1579 & 21.00 & 1 & 3.87 & -1.29\end{array}$

$\begin{array}{lllll}\text { A } 87 / 1579 & 21.00 & 1 & 3.87 & -1.29 \\ \text { A } 87 / 1580 & 21.00 & 1 & 3.48 & -1.09\end{array}$

$\begin{array}{lllll}\text { A } 87 / 1581 & 21.09 & 1 & 3.83 & -1.18\end{array}$

$\begin{array}{lllll}\text { A } 87 / 1582 & 21.09 & 4 & 4.65 & 1.42\end{array}$

$\begin{array}{lllll}\text { A } 87 / 1583 & 21.20 & 1 & 4.06 & -1.13\end{array}$

$\begin{array}{lllll}\text { A } 87 / 1584 & 21.30 & 1 & 4.30 & -1.37 \\ \text { A } 87 / 1585 & 21.30 & 3 & 3.43 & -0.36\end{array}$

$\begin{array}{lllll}\text { A } 87 / 1586 & 21.70 & 1 & 4.43 & -0.36 \\ \text { A } 87 / 1587 & 21.80 & 1 & 4.23 & -1.50\end{array}$

$\begin{array}{lllll}\text { A } 87 / 1587 & 21.80 & 1 & 4.23 & -1.21\end{array}$

$\begin{array}{lllll}\text { A } 87 / 1588 & 21.90 & 1,2 & 4.00 & -1.52\end{array}$

$\begin{array}{lllll}\text { A } 87 / 1589 & 22.00 & 1 & 4.18 & -1.16 \\ \text { A } 87 / 1590 & 22.10 & 1 & 3.92 & -1.14\end{array}$

$\begin{array}{lllll}\text { A } 87 / 1590 & 22.10 & 1 & 3.92 & -1.14 \\ \text { A } 87 / 1591 & 22.20 & 1 & 3.71 & -0.87\end{array}$ 
Table 3 (continued).

\begin{tabular}{|c|c|c|c|c|}
\hline $\begin{array}{c}\text { Sample } \\
\text { code }\end{array}$ & $\begin{array}{l}\text { Depth }{ }^{\mathrm{a}} \\
\text { (mbsf) }\end{array}$ & Species $^{\mathrm{b}}$ & $\delta^{18} \mathrm{O}$ & $\delta^{13} \mathrm{C}$ \\
\hline \multicolumn{5}{|c|}{ Hole 677A (Cont.) } \\
\hline A $87 / 1593$ & 22.30 & 1 & 3.78 & -1.09 \\
\hline A $87 / 1594$ & 22.37 & 1 & 4.00 & -1.00 \\
\hline A $87 / 1595$ & 22.37 & 3,5 & 3.15 & -0.22 \\
\hline A $87 / 1595$ & 22.50 & 1 & 4.31 & -1.21 \\
\hline A $87 / 1596$ & 22.50 & 4 & 4.81 & 1.39 \\
\hline A $87 / 1597$ & 22.60 & 1 & 4.42 & -1.46 \\
\hline A $87 / 1598$ & 22.60 & 4 & 4.92 & 0.74 \\
\hline A $87 / 1599$ & 22.60 & 3 & 3.96 & -0.59 \\
\hline A $87 / 1600$ & 22.67 & 1 & 5.00 & -1.32 \\
\hline A $87 / 1601$ & 22.67 & 3 & 4.10 & -0.65 \\
\hline A $87 / 1608$ & 22.80 & 2 & 5.18 & -1.77 \\
\hline A $87 / 1609$ & 22.85 & 1 & 5.20 & -1.39 \\
\hline A $87 / 1610$ & 22.85 & 3 & 4.25 & -0.80 \\
\hline A $87 / 1624$ & 23.00 & 2 & 4.77 & -1.95 \\
\hline A $87 / 1625$ & 23.10 & 1 & 4.95 & -1.53 \\
\hline A $87 / 1626$ & 23.20 & 1 & 5.05 & -1.62 \\
\hline A $87 / 1627$ & 23.30 & 1 & 5.13 & -1.50 \\
\hline A $87 / 1628$ & 23.30 & 3 & 4.03 & -0.89 \\
\hline A $87 / 1629$ & 23.30 & 4 & 5.46 & 1.12 \\
\hline A $87 / 1630$ & 23.40 & 1 & 4.96 & -1.48 \\
\hline A $87 / 1741$ & 23.50 & 1 & 4.68 & -1.75 \\
\hline A $87 / 1742$ & 23.60 & 1 & 4.98 & -1.56 \\
\hline A $87 / 1743$ & 23.60 & 4 & 5.46 & 1.14 \\
\hline A $87 / 1744$ & 23.70 & 1 & 4.87 & -1.61 \\
\hline A $87 / 1745$ & 23.80 & 1 & 4.78 & -1.64 \\
\hline A $87 / 1746$ & 23.87 & 1 & 4.84 & -1.53 \\
\hline A $87 / 1747$ & 24.00 & 1 & 4.67 & -1.61 \\
\hline A $87 / 1748$ & 24.09 & 1 & 4.92 & -1.46 \\
\hline A $87 / 1750$ & 24.18 & 2 & 4.56 & -2.11 \\
\hline A $87 / 1751$ & 24.29 & 1 & 4.71 & -1.72 \\
\hline A $87 / 1752$ & 24.29 & 4 & 5.11 & 0.81 \\
\hline A $87 / 1753$ & 24.39 & 1 & 4.92 & -1.60 \\
\hline A $87 / 1754$ & 24.49 & 1 & 4.41 & -1.73 \\
\hline A $87 / 1755$ & 24.59 & 1 & 4.62 & -1.49 \\
\hline A $87 / 1756$ & 24.70 & 1 & 4.43 & -1.34 \\
\hline A $87 / 1757$ & 24.70 & 2 & 4.46 & -1.78 \\
\hline A $87 / 1758$ & 24.80 & 1 & 4.57 & -1.53 \\
\hline A $87 / 1759$ & 24.90 & 1 & 4.34 & -1.51 \\
\hline A $87 / 1771$ & 25.09 & 1 & 4.16 & -1.22 \\
\hline A $87 / 1772$ & 25.19 & 1,2 & 4.01 & -1.44 \\
\hline A $87 / 1774$ & 25.28 & 1 & 4.47 & -1.35 \\
\hline A $87 / 1773$ & 25.29 & 1 & 3.89 & -1.14 \\
\hline A $87 / 1775$ & 25.39 & 1 & 4.41 & -1.31 \\
\hline A $87 / 1776$ & 25.49 & 1 & 4.37 & -1.32 \\
\hline A $87 / 1777$ & 25.59 & 1 & 4.33 & -1.32 \\
\hline A $87 / 1778$ & 25.66 & 1 & 4.41 & -1.28 \\
\hline A $87 / 1779$ & 26.02 & 1 & 4.37 & -1.69 \\
\hline A $87 / 1780$ & 26.10 & 1 & 4.33 & -1.82 \\
\hline A $87 / 1791$ & 26.20 & 1 & 4.63 & -1.61 \\
\hline A $87 / 1792$ & 26.30 & 1 & 4.73 & -1.61 \\
\hline A $87 / 1793$ & 26.37 & 1 & 4.76 & -1.65 \\
\hline A $87 / 1794$ & 26.50 & 1 & 4.71 & -1.50 \\
\hline A $87 / 1795$ & 26.60 & 1 & 4.64 & -1.46 \\
\hline A $87 / 1796$ & 26.71 & 1 & 4.67 & -1.44 \\
\hline A $87 / 1797$ & 26.80 & 1 & 4.58 & -1.45 \\
\hline A $87 / 1798$ & 26.90 & 1 & 4.37 & -1.46 \\
\hline A $87 / 1799$ & 27.00 & 1 & 4.39 & -1.40 \\
\hline A $87 / 1800$ & 27.10 & 1 & 4.52 & -1.23 \\
\hline A $87 / 1801$ & 27.19 & 1 & 4.60 & -1.70 \\
\hline A $87 / 1802$ & 27.30 & 1 & 4.24 & -1.67 \\
\hline A $87 / 1803$ & 27.41 & 1 & 4.02 & -1.36 \\
\hline A $87 / 1804$ & 27.49 & 1 & 3.85 & -1.73 \\
\hline A $87 / 1805$ & 27.56 & 1 & 3.80 & -1.49 \\
\hline A $87 / 1806$ & 27.68 & 1 & 3.66 & -1.34 \\
\hline A $87 / 1887$ & 27.80 & 1 & 3.73 & -1.61 \\
\hline A $87 / 1888$ & 27.90 & 1 & 3.88 & -1.66 \\
\hline A $87 / 1889$ & 27.99 & 1 & 4.01 & -1.46 \\
\hline А $87 / 1890$ & 28.10 & 1 & 4.61 & -1.54 \\
\hline A $87 / 1811$ & 28.20 & 1 & 4.58 & -1.68 \\
\hline A $87 / 1812$ & 28.30 & 1 & 4.69 & -1.64 \\
\hline A $87 / 1813$ & 28.40 & 1 & 4.71 & -1.60 \\
\hline A $87 / 1814$ & 28.50 & 1 & 4.68 & -1.58 \\
\hline A $87 / 1815$ & 28.60 & 1 & 4.65 & -1.54 \\
\hline A $87 / 1816$ & 28.70 & 1 & 4.70 & -1.54 \\
\hline A $87 / 1817$ & 28.77 & 1 & 4.67 & -1.53 \\
\hline A $87 / 1818$ & 28.87 & 1 & 4.47 & -1.47 \\
\hline A $87 / 1819$ & 28.99 & 1 & 4.09 & -1.55 \\
\hline A $87 / 1820$ & 29.09 & 1 & 4.19 & -1.42 \\
\hline A $87 / 1821$ & 29.18 & 1 & 3.92 & -1.54 \\
\hline A $87 / 1822$ & 29.29 & 1 & 3.98 & -1.54 \\
\hline
\end{tabular}

Table 3 (continued).

\begin{tabular}{ccccc}
\hline $\begin{array}{c}\text { Sample } \\
\text { code }\end{array}$ & $\begin{array}{c}\text { Depth } \\
\text { (mbsf) }\end{array}$ & Species $^{\mathrm{b}}$ & $\delta^{18} \mathrm{O}$ & $\delta^{13} \mathrm{C}$ \\
\hline Hole 677A (Cont.)
\end{tabular}

Table 3 (continued).

\begin{tabular}{|c|c|c|c|c|c|c|c|c|c|}
\hline $\begin{array}{c}\text { Sample } \\
\text { code }\end{array}$ & $\begin{array}{c}\text { Depth }{ }^{\mathrm{a}} \\
\text { (mbsf) }\end{array}$ & Species $^{\mathrm{b}}$ & $\delta^{18} \mathrm{O}$ & ${ }_{\delta}^{13} \mathrm{C}$ & $\begin{array}{l}\text { Sample } \\
\text { code }\end{array}$ & $\begin{array}{l}\text { Depth }{ }^{a} \\
\text { (mbsf) }\end{array}$ & Species $^{b}$ & $\delta^{18} \mathrm{O}$ & $\delta^{13} \mathrm{C}$ \\
\hline \multicolumn{5}{|c|}{ Hole 677A (Cont.) } & \multicolumn{5}{|c|}{ Hole 677A (Cont.) } \\
\hline A $87 / 1823$ & 29.40 & 1 & 4.14 & -1.72 & A $87 / 2135$ & 38.90 & 1,2 & 3.85 & -1.49 \\
\hline A $87 / 1824$ & 29.50 & 1 & 4.04 & -1.83 & A $87 / 2136$ & 39.00 & 1,2 & 3.91 & -1.50 \\
\hline A $87 / 1825$ & 29.60 & 1 & 4.20 & -1.45 & A $87 / 2137$ & 39.10 & 1,2 & 4.01 & -1.17 \\
\hline A $87 / 1826$ & 29.70 & $i$ & 4.06 & -1.61 & A $87 / 2138$ & 39.20 & 1 & 4.05 & -1.36 \\
\hline A $87 / 1827$ & 29.80 & 1 & 4.06 & -1.65 & A $87 / 2139$ & 39.30 & 1 & 4.35 & -1.29 \\
\hline A $87 / 1828$ & 30.00 & 1 & 4.12 & -1.63 & A $87 / 2140$ & 39.40 & 1 & 4.47 & -1.50 \\
\hline A $87 / 1829$ & 30.10 & 1 & 3.98 & -1.49 & A $87 / 2204$ & 39.48 & 1 & 4.62 & -1.46 \\
\hline A $87 / 1830$ & 30.20 & 1 & 3.86 & -1.54 & A $87 / 2205$ & 39.60 & 1 & 4.47 & -1.70 \\
\hline A $87 / 1831$ & 30.30 & 1 & 3.87 & -1.33 & A $87 / 2206$ & 39.70 & 1,2 & 4.59 & -1.61 \\
\hline A $87 / 1833$ & 30.40 & $i$ & 3.82 & -1.21 & A $87 / 2208$ & 39.80 & 1,2 & 4.19 & -1.94 \\
\hline A $87 / 1834$ & 30.50 & 1 & 3.71 & -1.20 & A $87 / 2209$ & 39.86 & 1,2 & 4.30 & -1.76 \\
\hline A $87 / 1835$ & 30.59 & 1 & 3.97 & -1.52 & A $87 / 2210$ & 40.00 & 1,2 & 4.27 & -1.61 \\
\hline A $87 / 1836$ & 30.68 & 1 & 3.89 & -1.28 & A $88 / 523$ & 40.10 & 1 & 4.37 & -1.53 \\
\hline A $87 / 1837$ & 30.80 & 1 & 3.71 & -1.46 & A $88 / 522$ & 40.18 & I & 4.36 & -1.46 \\
\hline A $87 / 1838$ & 30.90 & $i$ & 3.65 & -1.36 & A $88 / 521$ & 40.27 & 1 & 4.37 & -1.29 \\
\hline A $87 / 1839$ & 30.90 & 2 & 3.49 & -2.03 & A $88 / 520$ & 40.40 & 1 & 4.42 & -1.26 \\
\hline A $87 / 1840$ & 31.00 & 1 & 4.01 & -1.46 & A $88 / 519$ & 40.50 & 1 & 3.69 & -1.30 \\
\hline A $87 / 1841$ & 31.10 & 1 & 3.64 & -1.51 & A $88 / 518$ & 40.60 & 1 & 3.57 & -1.11 \\
\hline A $87 / 1842$ & 31.20 & $i$ & 4.18 & -1.64 & A $88 / 517$ & 40.70 & 1 & 3.48 & -1.19 \\
\hline A $87 / 1843$ & 31.30 & 1 & 4.49 & -1.80 & A $88 / 516$ & 40.80 & 1 & 3.44 & -1.13 \\
\hline A $87 / 1844$ & 31.40 & 1 & 4.62 & -1.45 & A $88 / 515$ & 40.90 & 1 & 3.43 & -1.13 \\
\hline A $87 / 1845$ & 31.50 & 1 & 5.09 & -1.61 & A $88 / 514$ & 41.00 & 1 & 3.45 & -1.11 \\
\hline A $87 / 1846$ & 31.60 & 1 & 4.99 & -1.60 & A $88 / 513$ & 41.10 & 1 & 3.65 & -1.17 \\
\hline A $87 / 1847$ & 31.70 & $i$ & 5.04 & -1.67 & A $88 / 512$ & 41.20 & 1 & 3.74 & -1.12 \\
\hline A $87 / 1848$ & 31.80 & 1 & 4.86 & -1.64 & A $88 / 511$ & 41.30 & 1 & 3.85 & -1.18 \\
\hline A $87 / 1849$ & 31.92 & 1 & 4.76 & -1.67 & A $88 / 510$ & 41.37 & 1 & 4.06 & -1.35 \\
\hline A $87 / 1850$ & 32.00 & 1 & 4.75 & -1.67 & A $88 / 509$ & 41.50 & 1,2 & 4.07 & -1.56 \\
\hline A $87 / 1851$ & 32.10 & 1 & 4.75 & -1.58 & A $88 / 508$ & 41.60 & 2 & 4.06 & -2.05 \\
\hline A $87 / 1852$ & 32.18 & 1 & 4.74 & -1.65 & A $88 / 507$ & 41.68 & 1 & 4.27 & -1.32 \\
\hline A $87 / 1853$ & 32.30 & 1 & 4.74 & -1.47 & A $88 / 506$ & 41.77 & 2 & 4.13 & -2.02 \\
\hline A $87 / 1854$ & 32.40 & 1 & 4.60 & -1.47 & A $88 / 505$ & 41.90 & 2 & 4.18 & -2.11 \\
\hline A $87 / 1855$ & 32.50 & $i$ & 4.37 & -1.62 & A $88 / 504$ & 42.00 & 2 & 4.26 & -1.93 \\
\hline A $87 / 1856$ & 32.60 & 1 & 4.37 & -1.67 & A $88 / 503$ & 42.10 & 1 & 4.43 & -1.40 \\
\hline A $87 / 1857$ & 32.70 & 1 & 4.21 & -1.74 & A $88 / 502$ & 42.20 & 1 & 4.22 & -1.21 \\
\hline A $87 / 1858$ & 32.80 & 1 & 4.02 & -1.70 & A $88 / 501$ & 42.30 & 1 & 4.04 & -1.14 \\
\hline A $87 / 1859$ & 32.90 & 1 & 4.26 & -1.68 & A $88 / 500$ & 42.40 & 1 & 3.87 & -1.16 \\
\hline A $87 / 1860$ & 33.00 & 1 & 4.21 & -1.91 & A $88 / 498$ & 42.50 & 1 & 3.76 & -1.33 \\
\hline A $87 / 2041$ & 33.10 & $i$ & 4.48 & -1.72 & A $88 / 499$ & 42.50 & 5 & 3.32 & -0.91 \\
\hline A $87 / 2042$ & 33.30 & 1 & 4.59 & -1.88 & A $88 / 497$ & 42.60 & 1 & 3.80 & -1.43 \\
\hline A $87 / 2043$ & 33.40 & 1 & 4.56 & -1.71 & A $88 / 496$ & 42.70 & 1 & 4.26 & -1.24 \\
\hline A $87 / 2044$ & 33.50 & 1 & 4.40 & -1.59 & A $88 / 495$ & 42.80 & 1 & 4.26 & -1.61 \\
\hline A $87 / 2045$ & 33.60 & 1 & 4.43 & -1.81 & A $88 / 494$ & 42.86 & 1 & 4.62 & -1.50 \\
\hline A $87 / 2046$ & 33.68 & 1 & 4.20 & -1.29 & A $88 / 492$ & 43.00 & 3 & 3.56 & -0.93 \\
\hline A $87 / 2047$ & 33.80 & 1,2 & 4.26 & -1.92 & A $88 / 493$ & 43.00 & 1,2 & 4.33 & -1.84 \\
\hline A $87 / 2048$ & 33.90 & 1 & 4.20 & -1.52 & A $88 / 491$ & 43.10 & 1 & 4.69 & -1.55 \\
\hline A $87 / 2049$ & 34.00 & 1 & 4.18 & -1.59 & A $88 / 490$ & 43.18 & 1 & 4.61 & -1.52 \\
\hline A $87 / 2050$ & 34.10 & 1 & 4.20 & -1.37 & A $88 / 489$ & 43.27 & 1 & 4.61 & -1.60 \\
\hline A $87 / 2101$ & 35.10 & 1 & 4.34 & -1.87 & A $88 / 488$ & 43.40 & 4 & 5.11 & 1.02 \\
\hline A $87 / 2102$ & 35.50 & 1 & 4.37 & -1.47 & A $88 / 487$ & 43.50 & 10 & 4.42 & -1.38 \\
\hline A $87 / 2103$ & 35.60 & 1,2 & 3.92 & -1.62 & A $88 / 486$ & 43.60 & 3 & 3.66 & -0.63 \\
\hline A $87 / 2104$ & 35.70 & 1 & 3.89 & -1.50 & A $88 / 485$ & 43.70 & 1,11 & 4.50 & -1.39 \\
\hline A $87 / 2105$ & 35.90 & 1 & 3.97 & -1.33 & A $88 / 484$ & 43.80 & 1 & 4.18 & -1.54 \\
\hline A $87 / 2106$ & 36.00 & 1,2 & 3.65 & -1.48 & A $88 / 483$ & 43.90 & 1 & 4.04 & -1.49 \\
\hline A $87 / 2107$ & 36.10 & 3 & 3.02 & -0.25 & A $88 / 482$ & 44.00 & 1 & 4.17 & -1.43 \\
\hline A $87 / 2108$ & 36.21 & 1 & 3.56 & -1.33 & A $88 / 481$ & 44.30 & 1 & 3.95 & -1.14 \\
\hline A $87 / 2109$ & 36.30 & 1 & 3.84 & -1.23 & A $88 / 480$ & 44.40 & 1 & 3.86 & -1.06 \\
\hline A $87 / 2110$ & 36.40 & 1 & 3.54 & -1.44 & A $88 / 479$ & 44.50 & 1 & 3.74 & -1.14 \\
\hline A $87 / 2111$ & 36.50 & 1 & 3.82 & -1.59 & A $88 / 478$ & 44.60 & 1 & 4.51 & -0.98 \\
\hline A $87 / 2112$ & 36.60 & 1 & 4.05 & -1.55 & A $88 / 524$ & 44.60 & 3 & 2.95 & -0.10 \\
\hline A $87 / 2113$ & 36.70 & i & 4.55 & -1.72 & A $88 / 525$ & 44.60 & 10 & 3.92 & -0.93 \\
\hline A $87 / 2114$ & 36.80 & i & 4.53 & -1.75 & A $88 / 477$ & 44.70 & 1 & 3.71 & -0.96 \\
\hline A $87 / 2115$ & 36.87 & i & 4.33 & -1.31 & A $88 / 476$ & 44.80 & 1 & 3.72 & -1.00 \\
\hline A $87 / 2116$ & 37.00 & 1 & 4.19 & -1.35 & A $88 / 475$ & 44.90 & 1 & 3.96 & -0.88 \\
\hline A $87 / 2117$ & 37.10 & 1,2 & 4.27 & -1.73 & A $88 / 456$ & 45.02 & 1 & 3.96 & -0.91 \\
\hline A $87 / 2118$ & 37.18 & $i$ & 4.09 & -1.43 & A $88 / 474$ & 45.10 & 1 & 3.98 & -1.03 \\
\hline A $87 / 2119$ & 37.28 & 1 & 4.07 & -1.40 & A $88 / 473$ & 45.18 & 1 & 4.01 & -1.00 \\
\hline A $87 / 2120$ & 37.40 & $i$ & 4.14 & -1.53 & A $88 / 472$ & 45.30 & 1 & 4.35 & -1.48 \\
\hline A $87 / 2121$ & 37.50 & 1,2 & 4.20 & -1.68 & A $88 / 470$ & 45,40 & $i$ & 4.05 & -0.92 \\
\hline A $87 / 2122$ & 37.60 & 1 & 4.00 & -1.49 & A $88 / 471$ & 45.40 & 3 & 3.23 & -0.01 \\
\hline A $87 / 2123$ & 37.71 & 1 & 4.24 & -1.34 & A $88 / 469$ & 45.50 & 3 & 3.51 & -0.18 \\
\hline A $87 / 2124$ & 37.80 & 1,2 & 4.13 & -1.47 & A $88 / 467$ & 45.60 & 3 & 3.21 & -0.22 \\
\hline A $87 / 2125$ & 37.90 & 1 & 4.09 & -1.30 & A $88 / 468$ & 45.60 & 2,11 & 3.96 & -1.28 \\
\hline A $87 / 2126$ & 38.00 & 1,2 & 3.86 & -1.39 & A $88 / 466$ & 45.71 & 3 & 3.24 & -0.41 \\
\hline A $87 / 2128$ & 38.20 & 1,2 & 4.30 & -1.50 & A $88 / 465$ & 45.80 & 1,2 & 4.04 & -2.03 \\
\hline A $87 / 2129$ & 38.31 & $1,2,9$ & 4.10 & -0.47 & A $88 / 464$ & 45.90 & 3,5 & 3.75 & -0.68 \\
\hline A $87 / 2130$ & 38.42 & 1 & 4.31 & -1.31 & A $88 / 463$ & 46.00 & 1,2 & 4.68 & -1.55 \\
\hline A $87 / 2131$ & 38.50 & $1,2,9$ & 4.17 & -0.20 & A $88 / 461$ & 46.10 & 1 & 4.58 & -1.48 \\
\hline A $87 / 2132$ & 38.60 & 1,2 & 4.03 & -1.47 & A $88 / 462$ & 46.10 & 3 & 3.85 & -0.57 \\
\hline A $87 / 2133$ & 38.68 & 1 & 3.90 & -1.49 & A $88 / 460$ & 46.20 & 1 & 4.53 & -1.54 \\
\hline A $87 / 2134$ & 38.77 & 1 & 4.02 & -1.19 & A $87 / 2207$ & 46.30 & 1 & 4.38 & -1.50 \\
\hline
\end{tabular}


Table 3 (continued).

\begin{tabular}{|c|c|c|c|c|}
\hline $\begin{array}{c}\text { Sample } \\
\text { code }\end{array}$ & $\begin{array}{l}\text { Depth } \\
\text { (mbsf) }\end{array}$ & Species $^{b}$ & $\delta^{18} \mathrm{O}$ & $\delta^{13} \mathrm{C}$ \\
\hline
\end{tabular}

\begin{tabular}{|c|c|c|c|c|}
\hline $88 / 459$ & 46.30 & 1,2 & 4.43 & -1.97 \\
\hline A $88 / 458$ & 46.40 & 1 & 4.39 & -1.57 \\
\hline A $88 / 457$ & 46.50 & 1 & 4.51 & -1.40 \\
\hline A $88 / 455$ & 46.60 & 1 & 4.38 & -1.35 \\
\hline A $88 / 454$ & 46.68 & 1 & 4.35 & -1.38 \\
\hline A $88 / 453$ & 46.80 & 1 & 3.88 & -1.18 \\
\hline A $88 / 451$ & 46.90 & 1 & 4.25 & -1.35 \\
\hline A $88 / 452$ & 47.00 & $i$ & 4.15 & -1.55 \\
\hline A $88 / 450$ & 47.10 & 1 & 3.96 & -1.20 \\
\hline A $88 / 449$ & 47.21 & 1 & 3.78 & -1.04 \\
\hline A $88 / 448$ & 47.30 & 1 & 3.54 & -0.99 \\
\hline A $88 / 447$ & 47.40 & i & 3.57 & -1.06 \\
\hline A $88 / 446$ & 47.50 & 1 & 3.58 & -1.02 \\
\hline A $88 / 445$ & 47.60 & i & 3.48 & -1.23 \\
\hline A $88 / 444$ & 47.70 & 1,2 & 3.42 & -1.10 \\
\hline A $88 / 443$ & 47.81 & $i$ & 3.46 & -1.31 \\
\hline A $88 / 442$ & 47.90 & 3,5 & 2.54 & -0.73 \\
\hline A $88 / 441$ & 48.00 & 3 & 3.07 & -0.66 \\
\hline A $88 / 440$ & 48.10 & 1 & 3.85 & -1.26 \\
\hline A $88 / 439$ & 48.20 & 1 & 4.07 & -1.71 \\
\hline A $88 / 438$ & 48.30 & 1 & 4.42 & -1.69 \\
\hline A $88 / 437$ & 48.80 & i & 3.99 & -1.76 \\
\hline A $88 / 436$ & 48.90 & 1 & 4.35 & -1.43 \\
\hline A $88 / 435$ & 49.00 & 1 & 4.21 & -1.55 \\
\hline A $88 / 434$ & 49.08 & 1 & 4.00 & -1.65 \\
\hline A $88 / 432$ & 49.19 & 1 & 3.97 & -1.50 \\
\hline A $88 / 433$ & 49.19 & 3 & 3.29 & -0.42 \\
\hline A $88 / 430$ & 49.39 & 1,2 & 3.83 & -1.39 \\
\hline A $88 / 429$ & 49.49 & 2 & 3.59 & -1.79 \\
\hline A $88 / 428$ & 49.59 & 1,2 & 3.86 & -1.19 \\
\hline A $88 / 427$ & 49.66 & 1,2 & 3.97 & -1.64 \\
\hline A $88 / 426$ & 49.80 & 1 & 4.40 & -1.47 \\
\hline A $88 / 425$ & 49.90 & i & 4.35 & -1.53 \\
\hline A $88 / 424$ & 50.00 & 1 & 4.30 & -1.57 \\
\hline A $88 / 423$ & 50.10 & i & 4.24 & -1.69 \\
\hline A $88 / 422$ & 50.21 & 1 & 4.43 & -1.53 \\
\hline A $88 / 420$ & 50.30 & $i$ & 4.40 & -1.56 \\
\hline A $88 / 421$ & 50.30 & 3,5 & 3.58 & -0.63 \\
\hline A $88 / 419$ & 50.40 & 1 & 4.28 & -1.59 \\
\hline A $88 / 418$ & 50.50 & 1 & 4.27 & -1.34 \\
\hline A $88 / 415$ & 50.60 & $i$ & 4.28 & -1.38 \\
\hline A $88 / 414$ & 50.70 & 1 & 4.28 & -1.37 \\
\hline A $88 / 240$ & 50.80 & 3 & 3.19 & -0.63 \\
\hline A $88 / 413$ & 50.80 & 1 & 4.03 & -1.43 \\
\hline A $88 / 412$ & 50.90 & i & 4.07 & -1.21 \\
\hline A $88 / 410$ & 51.00 & 3 & 2.90 & -0.30 \\
\hline A $88 / 411$ & 51.00 & 1 & 4.01 & -1.17 \\
\hline A $88 / 416$ & 51.10 & 3 & 3.08 & -0.14 \\
\hline A $88 / 417$ & 51.10 & 2,8 & 3.79 & -1.48 \\
\hline A $88 / 409$ & 51.17 & $1,2,8$ & 3.80 & -1.66 \\
\hline A $88 / 408$ & 51.30 & $i$ & 4.06 & -1.58 \\
\hline A $88 / 407$ & 51.40 & 1 & 4.09 & -1.51 \\
\hline A $88 / 406$ & 51.50 & 1 & 4.12 & -1.64 \\
\hline A $88 / 405$ & 51.60 & 1 & 4.30 & -1.64 \\
\hline A $88 / 404$ & 51.80 & i & 4.23 & -1.62 \\
\hline A $88 / 403$ & 51.90 & 1 & 4.34 & -1.70 \\
\hline A $88 / 402$ & 52.00 & 1 & 4.32 & -1.63 \\
\hline A $88 / 401$ & 52.10 & 1 & 4.38 & -1.56 \\
\hline A $88 / 400$ & 52.20 & i & 4.23 & -1.55 \\
\hline A $88 / 399$ & 52.30 & 1 & 4.21 & -1.54 \\
\hline A $88 / 398$ & 52.40 & 1 & 4.10 & -1.4 \\
\hline A $88 / 397$ & 52.50 & 1 & 3.74 & -1.26 \\
\hline A $88 / 396$ & 52.60 & $i$ & 3.62 & -1.31 \\
\hline A $88 / 395$ & 52.67 & 1 & 3.44 & -1.2 \\
\hline A $88 / 394$ & 52.80 & 1 & 3.57 & -1.07 \\
\hline A $88 / 393$ & 52.90 & 1 & 3.69 & -1.46 \\
\hline A $88 / 392$ & 53.00 & $i$ & 3.49 & -1.22 \\
\hline A $88 / 391$ & 53.10 & 1 & 3.63 & -1.4 \\
\hline A $88 / 390$ & 53.30 & 1 & 4.14 & \\
\hline A $88 / 389$ & 53.40 & 1 & 4.02 & -1.5 \\
\hline A $88 / 388$ & 53.48 & i & 4.28 & -1.47 \\
\hline A $88 / 387$ & 53.60 & 1 & 4.36 & \\
\hline A $88 / 386$ & 53.70 & 1 & 4.34 & \\
\hline A $88 / 385$ & 53.79 & $i$ & 4.43 & -1.55 \\
\hline A $88 / 384$ & 53.80 & 1 & 3.75 & -1.14 \\
\hline A $88 / 383$ & 53.90 & 1 & 3.73 & \\
\hline A $88 / 382$ & 54.00 & 3 & 2.96 & \\
\hline A $88 / 381$ & 54.10 & $1,2,8$ & 3.65 & -1.16 \\
\hline A $88 / 329$ & 54.20 & 3,5 & 3.11 & -0.49 \\
\hline
\end{tabular}

Table 3 (continued).

\begin{tabular}{|c|c|c|c|c|}
\hline $\begin{array}{l}\text { Sample } \\
\text { code }\end{array}$ & $\begin{array}{l}\text { Depth } \\
\text { (mbsf) }\end{array}$ & Species $^{b}$ & $\delta^{18} \mathrm{O}$ & $\delta^{13} \mathrm{C}$ \\
\hline
\end{tabular}

Table 3 (continued).

\begin{tabular}{|c|c|c|c|c|}
\hline $\begin{array}{l}\text { Sample } \\
\text { code }\end{array}$ & $\begin{array}{l}\text { Depth }^{\mathrm{a}} \\
\text { (mbsf) }\end{array}$ & Species $^{\mathrm{b}}$ & $\delta^{18} \mathrm{O}$ & $\delta^{13} \mathrm{C}$ \\
\hline \multicolumn{5}{|c|}{ Hole 677A (Cont.) } \\
\hline A $88 / 237$ & 61.10 & 1 & 3.97 & -1.28 \\
\hline A $88 / 236$ & 61.20 & 1 & 3.90 & -1.30 \\
\hline A $88 / 234$ & 61.30 & 3 & 2.86 & -0.50 \\
\hline A $88 / 235$ & 61.30 & 1,2 & 3.66 & -1.50 \\
\hline A $88 / 233$ & 61.40 & 3 & 2.69 & -0.62 \\
\hline A $88 / 231$ & 61.50 & 1 & 4.05 & -1.43 \\
\hline A $88 / 232$ & 61.50 & 2 & 3.75 & -1.77 \\
\hline A $88 / 230$ & 61.60 & 1 & 4.32 & -1.42 \\
\hline A $88 / 229$ & 61.70 & 1 & 4.45 & -1.49 \\
\hline A $88 / 227$ & 61.80 & 3,5 & 3.54 & -0.42 \\
\hline A $88 / 228$ & 61.80 & 2 & 4.24 & -1.70 \\
\hline A $88 / 226$ & 61.90 & 1 & 4.26 & -1.48 \\
\hline A $88 / 225$ & 62.00 & 3 & 3.37 & -0.54 \\
\hline A $88 / 224$ & 62.10 & 3 & 3.29 & -0.52 \\
\hline A $88 / 222$ & 62.18 & 1,2 & 3.79 & -1.45 \\
\hline A $88 / 223$ & 62.18 & 3 & 3.08 & -0.18 \\
\hline A $88 / 221$ & 62.30 & 1,2 & 3.79 & -1.30 \\
\hline A $88 / 220$ & 62.40 & 3 & 3.23 & -0.30 \\
\hline A $88 / 219$ & 62.50 & 1 & 3.80 & -1.00 \\
\hline A $88 / 218$ & 62.60 & 1 & 3.61 & -1.12 \\
\hline A $88 / 217$ & 62.70 & 3 & 2.85 & -0.13 \\
\hline A $88 / 215$ & 62.80 & 2 & $c_{4.36}$ & -1.69 \\
\hline A $88 / 216$ & 62.80 & 3 & 2.76 & -0.58 \\
\hline A $88 / 213$ & 62.90 & 1 & 3.81 & -0.86 \\
\hline A $88 / 214$ & 62.90 & 3 & 3.00 & -0.19 \\
\hline A $88 / 212$ & 63.00 & 3,5 & 3.15 & -0.31 \\
\hline A $88 / 211$ & 63.10 & 3 & 3.22 & -0.22 \\
\hline A $88 / 210$ & 63.18 & 3 & 3.25 & -0.54 \\
\hline A $88 / 209$ & 63.30 & 3 & 3.53 & -0.67 \\
\hline A $88 / 207$ & 63.40 & 3 & 3.67 & -0.79 \\
\hline A $88 / 208$ & 63.40 & 6 & 3.85 & -1.21 \\
\hline A $88 / 206$ & 63.50 & 3 & 3.62 & -0.71 \\
\hline A $88 / 205$ & 63.60 & 3 & 3.71 & -0.64 \\
\hline A $88 / 204$ & 63.70 & 3,5 & 3.61 & -0.75 \\
\hline A $88 / 203$ & 63.80 & 3 & 3.64 & -0.65 \\
\hline A $88 / 201$ & 63.90 & 3 & 3.42 & -0.48 \\
\hline A $88 / 202$ & 63.90 & 1,2 & 4.00 & -1.65 \\
\hline A $88 / 199$ & 64.00 & 1 & 4.09 & -1.07 \\
\hline A $88 / 200$ & 64.00 & 11 & 4.36 & -0.66 \\
\hline A $88 / 197$ & 64.10 & 1 & 4.04 & -1.23 \\
\hline A $88 / 198$ & 64.10 & 3 & 3.19 & -0.34 \\
\hline A $88 / 195$ & 64.18 & 3 & 3.15 & -0.42 \\
\hline A $88 / 196$ & 64.18 & 1 & 3.82 & -1.20 \\
\hline A $88 / 194$ & 64.30 & 3 & 3.20 & -0.38 \\
\hline A $88 / 193$ & 64.40 & 3 & 3.14 & -0.44 \\
\hline A $88 / 192$ & 64.50 & 3 & 3.09 & -0.55 \\
\hline A $88 / 191$ & 64.60 & 1 & 4.16 & -1.25 \\
\hline A $88 / 189$ & 64.70 & 1,8 & 4.03 & -1.43 \\
\hline A $88 / 190$ & 64.70 & 3 & 3.19 & -0.60 \\
\hline A $88 / 187$ & 64.80 & 3 & 3.33 & -0.51 \\
\hline A $88 / 188$ & 64.80 & 1,11 & 4.06 & -0.98 \\
\hline A $88 / 185$ & 64.90 & 1,8 & 4.04 & -1.44 \\
\hline A $88 / 186$ & 64.90 & 3 & 3.25 & -0.63 \\
\hline A $88 / 184$ & 65.00 & 1,8 & 4.11 & -1.42 \\
\hline A $88 / 183$ & 65.10 & 1 & 4.39 & -1.36 \\
\hline A $88 / 182$ & 65.20 & 1 & 4.49 & -1.48 \\
\hline A $88 / 181$ & 65.30 & 1 & 4.55 & -1.50 \\
\hline A $88 / 180$ & 65.41 & 1 & 4.36 & -1.30 \\
\hline A $88 / 179$ & 65.50 & 1 & 4.44 & -1.36 \\
\hline A $88 / 177$ & 65.60 & 1 & 4.18 & -1.14 \\
\hline A $88 / 178$ & 65.60 & 3 & 3.42 & -0.67 \\
\hline A $88 / 176$ & 65.68 & 1 & 4.22 & -1.36 \\
\hline A $88 / 175$ & 65.80 & 1 & 4.08 & -1.27 \\
\hline A $88 / 174$ & 65.90 & 1 & 3.88 & -1.37 \\
\hline A $88 / 173$ & 66.00 & 1 & 3.91 & -1.31 \\
\hline A $88 / 172$ & 66.10 & 1 & 4.04 & -1.36 \\
\hline A $88 / 171$ & 66.20 & 1 & 4.17 & -1.45 \\
\hline A $88 / 169$ & 66.31 & 1 & 4.20 & -1.35 \\
\hline A $88 / 170$ & 66.31 & 3 & 3.35 & -0.82 \\
\hline A $88 / 168$ & 66.40 & 1 & 4.00 & -1.41 \\
\hline A $88 / 166$ & 66.50 & 1 & 4.09 & -1.31 \\
\hline A $88 / 167$ & 66.50 & 2 & 4.09 & -1.57 \\
\hline A $88 / 165$ & 66.60 & 1 & 4.06 & -1.44 \\
\hline A $88 / 164$ & 66.70 & 2 & 3.83 & -1.72 \\
\hline A $88 / 163$ & 66.80 & 1 & 4.02 & -1.40 \\
\hline A $88 / 162$ & 66.90 & 1 & 4.00 & -1.56 \\
\hline A $88 / 160$ & 67.10 & 1 & 4.25 & -1.56 \\
\hline A $88 / 159$ & 67.18 & 1 & 4.47 & -1.22 \\
\hline A $88 / 158$ & 67.30 & 1 & 4.46 & -1.07 \\
\hline A $88 / 157$ & 67.40 & 1 & 4.55 & -0.96 \\
\hline
\end{tabular}


Table 3 (continued).

\begin{tabular}{|c|c|c|c|c|}
\hline $\begin{array}{l}\text { Sample } \\
\text { code }\end{array}$ & $\begin{array}{l}\text { Depth }{ }^{\mathrm{a}} \\
\text { (mbsf) }\end{array}$ & Species $^{b}$ & $\delta^{18} \mathrm{O}$ & $\delta^{13} \mathrm{C}$ \\
\hline \multicolumn{5}{|c|}{ Hole 677A (Cont.) } \\
\hline A $88 / 156$ & 67.55 & 1 & 4.09 & -1.31 \\
\hline A $88 / 155$ & 67.75 & 1 & 3.93 & -1.13 \\
\hline A $88 / 154$ & 67.80 & 5 & 2.96 & -0.41 \\
\hline A $88 / 153$ & 67.95 & 1 & 3.88 & -1.11 \\
\hline A $88 / 1040$ & 68.10 & 2,10 & 3.44 & -1.88 \\
\hline A $88 / 1039$ & 68.25 & 3,5 & 2.91 & -0.55 \\
\hline A $88 / 151$ & 68.30 & 3 & $c^{4} 4.82$ & 0.38 \\
\hline A 88/991 & 68.40 & 3 & 2.93 & -0.59 \\
\hline A $88 / 992$ & 68.50 & 1 & 3.85 & -1.40 \\
\hline A $88 / 993$ & 68.50 & 3 & 2.97 & -0.75 \\
\hline A $88 / 994$ & 68.60 & 1 & 3.96 & -1.74 \\
\hline A $88 / 995$ & 68.68 & 1 & 4.03 & -1.57 \\
\hline A $88 / 996$ & 68.68 & 3 & 3.21 & -0.94 \\
\hline A $88 / 997$ & 68.80 & 1 & 4.11 & -1.41 \\
\hline A $88 / 998$ & 68.90 & 1 & 4.15 & -1.54 \\
\hline A $88 / 999$ & 69.00 & 3 & 3.59 & -1.03 \\
\hline A $88 / 1000$ & 69.10 & 1 & 4.21 & -1.77 \\
\hline A $88 / 1001$ & 69.20 & 1 & 4.37 & -1.73 \\
\hline A $88 / 1002$ & 69.30 & 1 & 4.35 & -1.56 \\
\hline A $88 / 1003$ & 69.30 & 3 & 3.50 & -1.06 \\
\hline A $88 / 1004$ & 69.40 & 1 & 4.21 & -1.45 \\
\hline A $88 / 1005$ & 69.50 & 1,2 & 4.25 & -1.59 \\
\hline A $88 / 1007$ & 69.60 & 1 & 4.05 & -1.37 \\
\hline A $88 / 1009$ & 69.70 & 1 & 4.04 & -1.38 \\
\hline A $88 / 1009$ & 69.80 & 1 & 3.65 & -1.57 \\
\hline A $88 / 1010$ & 69.90 & 1 & 3.89 & -1.25 \\
\hline A $88 / 1011$ & 70.00 & 1 & 3.84 & -1.09 \\
\hline A $88 / 1012$ & 70.00 & 3 & 3.00 & -0.69 \\
\hline A $88 / 1013$ & 70.10 & 1 & 3.78 & -1.20 \\
\hline A $88 / 1014$ & 70.18 & $1,2,11$ & 3.93 & -1.15 \\
\hline A $88 / 1015$ & 70.30 & 1,2 & 4.10 & -1.24 \\
\hline A $88 / 1006$ & 70.40 & 5 & 3.24 & -0.64 \\
\hline A $88 / 1016$ & 70.40 & 4 & 4.33 & 1.30 \\
\hline A $88 / 1017$ & 70.50 & 3,5 & 3.08 & -0.63 \\
\hline A $88 / 1018$ & 70.60 & 1 & 3.82 & -1.67 \\
\hline A $88 / 1019$ & 70.70 & 1 & 3.88 & -1.53 \\
\hline A $88 / 1020$ & 70.80 & 1 & 4.03 & -1.62 \\
\hline A $88 / 1021$ & 70.80 & 3 & 3.24 & -0.77 \\
\hline A $88 / 1022$ & 70.90 & 1 & 4.28 & -1.43 \\
\hline A $88 / 1023$ & 70.90 & 3 & 3.07 & -0.96 \\
\hline A $88 / 1024$ & 71.00 & 1 & 4.16 & -1.62 \\
\hline A $88 / 1025$ & 71.10 & 1 & 4.07 & -1.54 \\
\hline A $88 / 1026$ & 71.10 & 3 & 3.29 & -0.92 \\
\hline A $88 / 1027$ & 71.20 & 1,2 & 4.02 & -1.44 \\
\hline A $88 / 1028$ & 71.20 & 5 & 3.40 & -0.77 \\
\hline A $88 / 1029$ & 71.30 & 4 & 4.54 & 1.27 \\
\hline A $88 / 1030$ & 71.30 & 1,11 & 4.09 & -0.76 \\
\hline A $88 / 1031$ & 71.40 & 1,11 & 3.85 & -1.10 \\
\hline A $88 / 1032$ & 71.50 & 3 & 2.96 & -0.75 \\
\hline A $88 / 1033$ & 71.50 & 4 & 4.37 & 1.04 \\
\hline A $88 / 1034$ & 71.60 & 1 & 4.00 & -1.47 \\
\hline A $88 / 1035$ & 71.60 & 3 & 3.18 & -0.86 \\
\hline A $88 / 1036$ & 71.68 & 1 & 3.77 & -1.54 \\
\hline A $88 / 1037$ & 71.80 & 3,5 & 3.14 & -0.50 \\
\hline A $88 / 1038$ & 71.90 & 1 & 4.00 & -1.21 \\
\hline A $88 / 1041$ & 72.00 & 1 & 4.19 & -1.48 \\
\hline A $88 / 1042$ & 72.05 & 3 & 3.31 & -0.42 \\
\hline A $88 / 1043$ & 72.10 & 4 & 4.10 & 0.94 \\
\hline A $88 / 1044$ & 72.20 & 4 & 4.53 & 1.19 \\
\hline A $88 / 1045$ & 72.20 & 3 & 3.09 & -0.49 \\
\hline A $88 / 1046$ & 72.30 & 1 & 4.49 & -0.74 \\
\hline A $88 / 1047$ & 72.40 & 8,11 & 4.30 & -1.39 \\
\hline A $88 / 1048$ & 72.50 & 1,9 & 3.98 & -0.57 \\
\hline A $88 / 1049$ & 72.70 & 1 & 4.19 & -1.39 \\
\hline A $88 / 1051$ & 72.80 & 1 & 4.07 & -1.45 \\
\hline A $88 / 1052$ & 72.89 & $1,2,11$ & 4.12 & -1.22 \\
\hline A $88 / 1053$ & 73.00 & 2 & 3.86 & -1.29 \\
\hline A $88 / 1054$ & 73.10 & 1 & 3.92 & -1.27 \\
\hline A $88 / 1055$ & 73.20 & 1 & 3.79 & -1.44 \\
\hline A $88 / 1056$ & 73.28 & 1,11 & 3.86 & -1.20 \\
\hline A $88 / 1057$ & 73.40 & 3 & 3.02 & -0.69 \\
\hline A $88 / 1058$ & 73.49 & 2,11 & 3.77 & -1.34 \\
\hline A $88 / 1050$ & 73.59 & 3,5 & 3.20 & -0.50 \\
\hline A $88 / 1059$ & 73.68 & 1 & 3.93 & -1.65 \\
\hline A $88 / 1060$ & 73.79 & 3 & 2.87 & -0.83 \\
\hline A $88 / 1061$ & 73.89 & 1 & 3.86 & -1.44 \\
\hline A $88 / 1062$ & 74.00 & 1 & 4.00 & -1.62 \\
\hline A $88 / 1063$ & 74.09 & 1,2 & 4.31 & -1.58 \\
\hline A $88 / 1064$ & 74.20 & 1 & 4.27 & -1.66 \\
\hline A $88 / 1065$ & 74.20 & 2 & 4.15 & -1.84 \\
\hline
\end{tabular}

Table 3 (continued).

\begin{tabular}{ccccc}
\hline $\begin{array}{c}\text { Sample } \\
\text { code }\end{array}$ & $\begin{array}{c}\text { Depth } \\
\text { (mbsf) }\end{array}$ & Species $^{\mathrm{b}}$ & $\delta^{18} \mathrm{O}$ & $\delta^{13} \mathrm{C}$ \\
\hline Hole 677A (Cont.)
\end{tabular}

\begin{tabular}{|c|c|c|c|c|}
\hline A $88 / 1066$ & 74.29 & 2 & 4.32 & -1.73 \\
\hline A $88 / 1067$ & 74.37 & 1 & 4.20 & -1.64 \\
\hline A $88 / 1068$ & 74.48 & 1 & 4.19 & -1.39 \\
\hline A $88 / 1069$ & 74.59 & 1 & 4.30 & -1.27 \\
\hline A $88 / 1070$ & 74.69 & 1 & 4.13 & -1.47 \\
\hline A $88 / 1071$ & 74.78 & 1 & 4.00 & -1.35 \\
\hline A $88 / 1072$ & 74.89 & 1 & 3.93 & -1.54 \\
\hline A $88 / 1073$ & 74.99 & 1 & 3.87 & -1.32 \\
\hline A $88 / 1074$ & 75.09 & 1 & 3.80 & -1.18 \\
\hline A $88 / 1075$ & 75.18 & 1 & 3,96 & -1.65 \\
\hline A $88 / 1076$ & 75.30 & 1 & 3.65 & -1.27 \\
\hline A $88 / 1077$ & 75.40 & 1,2 & 3.52 & -1.41 \\
\hline A $88 / 1078$ & 75.50 & 1,2 & 3.54 & -1.26 \\
\hline A $88 / 1079$ & 75.60 & 3 & 2.69 & -0.75 \\
\hline A $88 / 1080$ & 75.70 & 1 & 3.70 & -1.40 \\
\hline A $88 / 1081$ & 75.80 & 1 & 3.70 & -1.36 \\
\hline A $88 / 1082$ & 75.89 & 1 & 3.95 & -1.50 \\
\hline A $88 / 1083$ & 76.00 & 3 & 3.29 & -0.63 \\
\hline A $88 / 1084$ & 76.00 & 1 & 4.23 & -1.50 \\
\hline A $88 / 1085$ & 76.10 & 1 & 4.26 & -1.64 \\
\hline A $88 / 1086$ & 76.20 & 1 & 4.25 & -1.52 \\
\hline A $88 / 1087$ & 76.27 & 1 & 4.22 & -1.53 \\
\hline A $88 / 1088$ & 76.40 & 3 & 3.21 & -0.88 \\
\hline A $88 / 1089$ & 76.40 & 1,2 & 3.89 & -1.74 \\
\hline A $88 / 1090$ & 76.50 & 1 & 4.23 & -1.37 \\
\hline A $88 / 1091$ & 76.60 & 3 & 3.30 & -0.74 \\
\hline A $88 / 1092$ & 76.70 & 3 & 3.27 & -0.72 \\
\hline A $88 / 1093$ & 76,80 & 1 & 3.77 & -1.40 \\
\hline A $88 / 1094$ & 77.20 & 1 & 3.70 & -1.32 \\
\hline A $88 / 1095$ & 77.30 & 1 & 3.65 & -1.15 \\
\hline A $88 / 1096$ & 77.39 & 1 & 3.63 & -1.08 \\
\hline A $88 / 1097$ & 77.50 & 1 & 3.53 & -1.24 \\
\hline A $88 / 1098$ & 77.60 & 1 & 3.49 & -1.41 \\
\hline A $88 / 1099$ & 77.70 & 1 & 3.42 & -1.47 \\
\hline A $88 / 1100$ & 77.78 & 2 & 3.42 & -1.27 \\
\hline A $88 / 1101$ & 77.78 & 8,11 & 3.64 & -1.67 \\
\hline A $88 / 1102$ & 77.90 & 1 & 3.54 & -1.11 \\
\hline A $88 / 1103$ & 77.90 & 3,5 & 2.63 & -0.25 \\
\hline A $88 / 1104$ & 78.00 & 3,5 & 2.64 & -0.61 \\
\hline A $88 / 1105$ & 78.00 & 2 & 3.45 & -1.30 \\
\hline A $88 / 1106$ & 78.10 & 2 & 3.58 & -1.34 \\
\hline A $88 / 1107$ & 78.18 & 2 & 3.76 & -1.16 \\
\hline A $88 / 1108$ & 78.18 & 3 & 2.77 & -0.40 \\
\hline A $88 / 1109$ & 78.30 & 3 & 2.97 & -0.41 \\
\hline A $88 / 1110$ & 78.30 & 2 & 3.76 & -1.22 \\
\hline A $88 / 1111$ & 78.40 & $1,2,11$ & 3.80 & -0.96 \\
\hline A $88 / 1112$ & 78.50 & 1,11 & 3.88 & -0.73 \\
\hline A $88 / 1113$ & 78.60 & 3,5 & 3.13 & -0.55 \\
\hline A $88 / 1114$ & 78.70 & 3 & 3.20 & -0.34 \\
\hline A $88 / 1115$ & 78.80 & 2,11 & 3.80 & -1.07 \\
\hline A $88 / 1116$ & 78.90 & 3,5 & 3.19 & -0.88 \\
\hline A $88 / 1117$ & 79.00 & 3 & 3.24 & -0.65 \\
\hline A $88 / 1118$ & 79.10 & 3 & 3.40 & -0.43 \\
\hline A $88 / 1119$ & 79.20 & 3 & 3.39 & -0.66 \\
\hline A $88 / 1120$ & 79.30 & 3 & 3.38 & -0.45 \\
\hline A $88 / 1121$ & 79.40 & 3 & 2.99 & -0.89 \\
\hline A $88 / 1122$ & 79.50 & 3 & 3.34 & -0.37 \\
\hline A $88 / 1123$ & 79.65 & 3 & 3.28 & -0.76 \\
\hline A $88 / 1124$ & 79.80 & 3 & 3.31 & -0.52 \\
\hline A $88 / 1125$ & 79.90 & 3 & 3.31 & -0.45 \\
\hline A $88 / 1126$ & 80.01 & 3 & 3.36 & -0.82 \\
\hline A $88 / 1127$ & 80.10 & 3 & 3.35 & -0.47 \\
\hline A $88 / 1128$ & 80.20 & 3 & 3.44 & -0.72 \\
\hline A $88 / 1129$ & 80.20 & 1,11 & 4.25 & -1.10 \\
\hline A $88 / 1130$ & 80.29 & 3 & 3.45 & -0.63 \\
\hline A $88 / 1131$ & 80.39 & 3 & 3.26 & -0.56 \\
\hline A $88 / 1132$ & 80.49 & 3 & 3.23 & -0.53 \\
\hline A $88 / 1133$ & 80.59 & 1,11 & 4.11 & -1.10 \\
\hline A $88 / 1134$ & 80.69 & 1,11 & 4.04 & -1.06 \\
\hline A $88 / 1135$ & 80.79 & 1 & 3.97 & -1.32 \\
\hline A $88 / 1136$ & 80.79 & 3 & 3.34 & -0.50 \\
\hline A $88 / 1137$ & 80.90 & 3 & 3.16 & -0.55 \\
\hline A $88 / 1138$ & 80.90 & 1,8 & 3.81 & -1.46 \\
\hline A $88 / 1139$ & 81.00 & 3 & 3.08 & -0.70 \\
\hline A $88 / 1140$ & 81.10 & 1 & 4.00 & -1.31 \\
\hline A $88 / 1141$ & 81.18 & 3 & 3.16 & -0.29 \\
\hline A $88 / 1142$ & 81.30 & 3 & 3.11 & -0.47 \\
\hline A $88 / 1143$ & 81.41 & 3 & 3.03 & -0.51 \\
\hline A $88 / 1144$ & 81.50 & 3 & 3.08 & -0.43 \\
\hline A $88 / 1145$ & 1.60 & 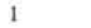 & 3.92 & -1.46 \\
\hline
\end{tabular}

Table 3 (continued).

\begin{tabular}{|c|c|c|c|c|}
\hline $\begin{array}{l}\text { Sample } \\
\text { code }\end{array}$ & $\begin{array}{l}\text { Depth }^{\mathrm{a}} \\
\text { (mbsf) }\end{array}$ & Species $^{\mathrm{b}}$ & $\delta^{18} \mathrm{O}$ & $\delta^{13} \mathrm{C}$ \\
\hline \multicolumn{5}{|c|}{ Hole 677A (Cont.) } \\
\hline A $88 / 1146$ & 82.30 & 8,11 & 4.27 & -1.80 \\
\hline A $88 / 1147$ & 82.50 & 8,11 & 4.06 & -2.47 \\
\hline A $88 / 1148$ & 82.60 & 1 & 3.90 & -1.75 \\
\hline A $88 / 1149$ & 82.60 & 8,11 & 4.20 & -2.21 \\
\hline A $88 / 1150$ & 82.70 & 1 & 4.07 & -1.59 \\
\hline A $88 / 1261$ & 82.90 & 1 & 4.18 & -1.63 \\
\hline A $88 / 1262$ & 83.00 & 1 & 4.39 & -1.48 \\
\hline A $88 / 1263$ & 83.10 & 1 & 4.23 & -1.55 \\
\hline A $88 / 1264$ & 83.10 & 3 & 3.62 & -0.92 \\
\hline A $88 / 1265$ & 83.18 & 1 & 4.45 & -1.53 \\
\hline A $88 / 1266$ & 83.30 & 1 & 4.49 & -1.59 \\
\hline A $88 / 1267$ & 83.40 & 1 & 4.78 & -1.46 \\
\hline A $88 / 1495$ & 83.40 & 3 & 3.61 & -0.89 \\
\hline A $88 / 1496$ & 83.40 & 8,11 & 4.49 & -2.49 \\
\hline A $88 / 1268$ & 83.50 & 1 & 4.44 & -1.81 \\
\hline A $88 / 1269$ & 83.60 & 1 & 4.49 & -1.56 \\
\hline A $88 / 1270$ & 83.70 & 1 & 4.41 & -1.83 \\
\hline A $88 / 1271$ & 83.80 & 1 & 4.40 & -1.71 \\
\hline A $88 / 1272$ & 83.89 & 1 & 4.24 & -1.68 \\
\hline A $88 / 1273$ & 83.89 & 3 & 3.37 & -1.15 \\
\hline A $88 / 1274$ & 84.00 & 1 & 4.34 & -1.55 \\
\hline A $88 / 1275$ & 84.10 & 1 & 4.25 & -1.66 \\
\hline A $88 / 1276$ & 84.20 & 1 & 4.20 & -1.66 \\
\hline A $88 / 1277$ & 84.29 & 1,2 & 4.18 & -1.63 \\
\hline A $88 / 1278$ & 84.40 & 1,2 & 4.45 & -1.26 \\
\hline A $88 / 1279$ & 84.50 & 1 & 4.23 & -1.63 \\
\hline A $88 / 1280$ & 84.60 & 1 & 4.32 & -1.48 \\
\hline A $88 / 1281$ & 84.68 & 1 & 4.10 & -1.43 \\
\hline A $88 / 1282$ & 84.80 & 1 & 3.97 & -1.49 \\
\hline A $88 / 1283$ & 84.89 & 1 & 3.95 & -1.56 \\
\hline A $88 / 1284$ & 85.00 & 1,9 & 3.95 & -1.25 \\
\hline A $88 / 1285$ & 85.10 & 1 & 3.80 & -1.54 \\
\hline A $88 / 1286$ & 85.20 & 1 & 3.89 & -1.59 \\
\hline A $88 / 1287$ & 85.30 & 1 & 3.76 & -1.60 \\
\hline A $88 / 1288$ & 85.39 & 1 & 3.77 & -1.61 \\
\hline A $88 / 1289$ & 85.50 & 1 & 3.83 & -1.57 \\
\hline A $88 / 1290$ & 85.60 & 1 & 3.71 & -1.45 \\
\hline A $88 / 1291$ & 85.70 & 1 & 3.80 & -1.44 \\
\hline A $88 / 1292$ & 85.79 & 1,2 & 3.69 & -1.68 \\
\hline A $88 / 1293$ & 85.90 & 1 & 3.93 & -1.36 \\
\hline A $88 / 1294$ & 86.00 & 3 & 2.96 & -0.39 \\
\hline A $88 / 1295$ & 86.10 & 2 & 3.71 & -1.58 \\
\hline A $88 / 1296$ & 86.18 & 2 & 3.66 & -1.50 \\
\hline A $88 / 1297$ & 86.30 & 1 & 4.38 & -1.19 \\
\hline A $88 / 1298$ & 86.40 & 4 & 4.58 & 1.15 \\
\hline A $88 / 1299$ & 86.50 & 2 & 4.01 & -1.67 \\
\hline A $88 / 1315$ & 86.60 & 1,2 & 4.24 & -1.49 \\
\hline A $88 / 1300$ & 86.70 & 1 & 3.70 & -1.33 \\
\hline A $88 / 1301$ & 86.80 & 1 & 3.59 & -1.26 \\
\hline A $88 / 1302$ & 86.89 & 2 & 3.38 & -1.86 \\
\hline A $88 / 1303$ & 87.00 & 2,8 & 3.47 & -1.54 \\
\hline A $88 / 1304$ & 87.10 & 2 & 3.48 & -1.90 \\
\hline A $88 / 1305$ & 87.20 & 1,2 & 3.65 & -1.79 \\
\hline A $88 / 1306$ & 87.29 & 1 & 4.37 & -1.84 \\
\hline A $88 / 1307$ & 87.39 & 1 & 4.44 & -1.76 \\
\hline A $88 / 1308$ & 87.50 & 1 & 4.54 & -1.83 \\
\hline A $88 / 1309$ & 87.60 & 1 & 4.44 & -1.97 \\
\hline A $88 / 1310$ & 87.68 & 1 & 4.47 & -1.87 \\
\hline A $88 / 1311$ & 87.80 & 1 & 4.30 & -1.81 \\
\hline A $88 / 1312$ & 87.90 & 1 & 4.36 & -1.68 \\
\hline A $88 / 1313$ & 88.00 & 1 & 4.26 & -1.82 \\
\hline A $88 / 1314$ & 88.10 & 1.2 & 4.24 & -1.63 \\
\hline A $88 / 1316$ & 88.20 & 1 & 4.09 & -1.61 \\
\hline A $88 / 1317$ & 88.30 & 1 & 4.00 & -1.59 \\
\hline A $88 / 1318$ & 88.39 & 1 & 3.94 & -1.45 \\
\hline A $88 / 1319$ & 88.50 & 1 & 3.82 & -1.55 \\
\hline A $88 / 1320$ & 88.60 & 1 & 3.68 & -1.47 \\
\hline A $88 / 1321$ & 88.70 & 3 & 3.00 & -0.61 \\
\hline A $88 / 1322$ & 88.70 & 1 & 3.76 & -1.52 \\
\hline A $88 / 1323$ & 88.79 & 1,8 & 3.60 & -1.29 \\
\hline A $88 / 1324$ & 88.90 & 3 & 3.03 & -0.45 \\
\hline A $88 / 1325$ & 89.00 & 3.5 & 3.02 & -0.65 \\
\hline A $88 / 1326$ & 89.10 & 3 & 3.05 & -0.49 \\
\hline A $88 / 1327$ & 89.10 & 1,2 & 3.86 & -1.57 \\
\hline A $88 / 1328$ & 89.18 & 1 & 3.92 & -1.83 \\
\hline A $88 / 1329$ & 89.30 & 1 & 4.16 & -1.60 \\
\hline A $88 / 1330$ & 89.40 & 1 & 4.13 & -1.55 \\
\hline A $88 / 1331$ & 89.50 & 1 & 4.07 & -1.62 \\
\hline A $88 / 1332$ & 89.60 & 1,2 & 3.99 & -1.92 \\
\hline A $88 / 1333$ & 89.70 & 12 & 4.15 & -2.19 \\
\hline
\end{tabular}


Table 3 (continued).

\begin{tabular}{|c|c|c|c|c|}
\hline $\begin{array}{l}\text { Sample } \\
\text { code }\end{array}$ & $\begin{array}{l}\text { Depth }^{\mathrm{a}} \\
\text { (mbsf) }\end{array}$ & Species $^{b}$ & $\delta^{18} \mathrm{O}$ & $\delta^{13} \mathrm{C}$ \\
\hline \multicolumn{5}{|c|}{ Hole 677A (Cont.) } \\
\hline A $88 / 1334$ & 89.80 & 12 & 4.14 & -2.18 \\
\hline A $88 / 1335$ & 89.89 & 12 & 4.06 & -2.51 \\
\hline A $88 / 1336$ & 90.00 & 12 & 4.22 & -2.04 \\
\hline A $88 / 1337$ & 90.10 & 2,8 & 4.04 & -1.91 \\
\hline A $88 / 1338$ & 90.20 & 12 & 3.93 & -2.27 \\
\hline A $88 / 1340$ & 90.29 & 12 & 3.99 & -2.30 \\
\hline A $88 / 1341$ & 90.40 & 3 & 2.75 & -0.84 \\
\hline A $88 / 1342$ & 90.50 & 1 & 3.95 & -1.57 \\
\hline A $88 / 1343$ & 90.60 & 1 & 3.74 & -1.46 \\
\hline A $88 / 1354$ & 90.67 & 1 & 3.74 & -1.67 \\
\hline A $88 / 1345$ & 90.80 & 3 & 2.95 & -0.47 \\
\hline A $88 / 1346$ & 90.90 & 3 & 2.68 & -0.72 \\
\hline A $88 / 1347$ & 91.00 & 2 & 3.62 & -1.81 \\
\hline A $88 / 1497$ & 91.10 & 2 & 3.67 & -1.69 \\
\hline A $88 / 1498$ & 91.10 & 8,11 & 3.60 & -3.00 \\
\hline A $88 / 1349$ & 91.20 & 3 & 2.64 & -0.64 \\
\hline A $88 / 1350$ & 91.30 & 1,2 & 4.13 & -1.82 \\
\hline A $88 / 1351$ & 91.39 & 3 & 3.26 & -0.98 \\
\hline A $88 / 1352$ & 91.50 & 1 & 4.24 & -1.74 \\
\hline A $88 / 1353$ & 91.60 & 1 & 4.24 & -1.51 \\
\hline A $88 / 1353$ & 91.70 & 1 & 4.10 & -1.57 \\
\hline A $88 / 1355$ & 91.70 & 2 & 4.11 & -2.08 \\
\hline A $88 / 1356$ & 91.80 & 1 & 4.27 & -1.74 \\
\hline A $88 / 1357$ & 91.90 & 1 & 4.07 & -1.69 \\
\hline A $88 / 1358$ & 92.00 & 1 & 3.92 & -1.84 \\
\hline A $88 / 1359$ & 92.10 & 2 & 4.13 & -1.86 \\
\hline A $88 / 1360$ & 92.20 & 2 & 4.17 & -1.85 \\
\hline A $88 / 1361$ & 92.30 & 3 & 3.08 & -0.62 \\
\hline A $88 / 1362$ & 92.40 & 3 & 2.83 & -0.33 \\
\hline A $88 / 1363$ & 92.40 & 1,2 & 3.57 & -1.71 \\
\hline A $88 / 1364$ & 92.50 & 3 & 2.92 & -0.48 \\
\hline A $88 / 1365$ & 92.60 & 2 & 3.48 & -2.21 \\
\hline A $88 / 1366$ & 92.68 & 3 & 2.92 & -0.54 \\
\hline A $88 / 1367$ & 92.80 & 3 & 2.84 & -0.55 \\
\hline A $88 / 1368$ & 92.90 & 3 & 2.88 & -0.58 \\
\hline A $88 / 1369$ & 92.90 & 1 & 3.80 & -1.55 \\
\hline A $88 / 1370$ & 93.00 & 3 & 2.64 & -0.95 \\
\hline A $88 / 1500$ & 93.00 & 8,11 & 3.90 & -2.39 \\
\hline A $88 / 1371$ & 93.10 & 1 & 3.90 & -1.43 \\
\hline A $88 / 1372$ & 93.20 & 1 & 3.81 & -1.49 \\
\hline A $88 / 1373$ & 93.30 & 1 & 3.96 & -1.52 \\
\hline A $88 / 1374$ & 93.30 & 3 & 3.06 & -0.56 \\
\hline A $88 / 1375$ & 93.40 & 1 & 4.03 & -1.63 \\
\hline A $88 / 1377$ & 93.50 & 3 & 2.67 & -1.00 \\
\hline A $88 / 1499$ & 93.50 & 8,11 & 3.95 & -2.64 \\
\hline A $88 / 1376$ & 93.60 & 1,2 & 3.72 & -1.98 \\
\hline A $88 / 1378$ & 93.70 & 3 & 2.93 & -0.69 \\
\hline A $88 / 1379$ & 93.80 & $1,2,8$ & 3.79 & -1.75 \\
\hline A $88 / 1380$ & 93.91 & 1 & 3.83 & -1.73 \\
\hline A $88 / 1381$ & 93.91 & 3 & 3.07 & -0.84 \\
\hline A $88 / 1382$ & 94.00 & 3,5 & 3.02 & -0.83 \\
\hline A $88 / 1383$ & 94.10 & 3,5 & 3.00 & -0.86 \\
\hline A $88 / 1384$ & 94.18 & $1,2,8$ & 3.44 & -1.88 \\
\hline A $88 / 1385$ & 94.30 & 3 & 2.90 & -0.50 \\
\hline A $88 / 1386$ & 94.40 & 3,5 & 2.80 & -0.50 \\
\hline A $88 / 1387$ & 94.50 & 9 & 3.49 & -0.26 \\
\hline A $88 / 1388$ & 94.60 & 3 & 2.69 & -0.31 \\
\hline A $88 / 1389$ & 94.60 & 12 & 3.59 & -2.57 \\
\hline A $88 / 1390$ & 94.70 & 3,5 & 2.57 & -0.92 \\
\hline A $88 / 1391$ & 94.80 & 3,5 & 3.34 & -0.64 \\
\hline A $88 / 1394$ & 94.90 & $1,2,10$ & 3.80 & -1.75 \\
\hline A $88 / 1392$ & 95.00 & 3,5 & 3.23 & -0.82 \\
\hline A $88 / 1393$ & 95.10 & 2, 10 & 4.05 & -1.57 \\
\hline A $88 / 1395$ & 95.20 & 3,5 & 3.12 & -0.69 \\
\hline A $88 / 1396$ & 95.30 & 1,2 & 3.98 & -1.77 \\
\hline A $88 / 1397$ & 95.30 & 3 & 3.09 & -0.67 \\
\hline A $88 / 1398$ & 95.41 & 2,10 & 3.58 & -1.92 \\
\hline A $88 / 1399$ & 95.50 & 2 & 3.74 & -1.69 \\
\hline A $88 / 1400$ & 95.60 & 10 & 3.93 & -1.38 \\
\hline A $88 / 1401$ & 95.68 & $1,2,12$ & 3.99 & -1.96 \\
\hline A $88 / 1402$ & 95.80 & 8,11 & 3.96 & -2.11 \\
\hline A $88 / 1403$ & 95.90 & 8,11 & 3.83 & -2.44 \\
\hline A $88 / 1404$ & 96.00 & 8,11 & 3.80 & -2.35 \\
\hline A $88 / 1405$ & 96.10 & $3,5,6$ & 3.15 & -0.89 \\
\hline A $88 / 1406$ & 96.20 & 8,11 & 3.71 & -2.44 \\
\hline A $88 / 1407$ & 96.30 & 3,6 & 2.95 & -0.60 \\
\hline A $88 / 1408$ & 96.30 & 12 & 3.90 & -2.04 \\
\hline A $88 / 1409$ & 96.40 & 5 & 3.19 & -0.56 \\
\hline A $88 / 1410$ & 96.50 & 12 & 3.96 & -2.30 \\
\hline A $88 / 1411$ & 96.60 & 3 & 2.89 & -0.64 \\
\hline
\end{tabular}

Table 3 (continued).

\begin{tabular}{lllll}
\hline $\begin{array}{c}\text { Sample } \\
\text { code }\end{array}$ & $\begin{array}{c}\text { Depth } \\
\text { (mbsf) }\end{array}$ & Species $^{\mathrm{b}}$ & $\delta^{18} \mathrm{O}$ & $\delta^{13} \mathrm{C}$ \\
\hline Hole 677A (Cont.)
\end{tabular}

Table 3 (continued).

\begin{tabular}{|c|c|c|c|c|c|c|c|c|c|}
\hline $\begin{array}{l}\text { Sample } \\
\text { code }\end{array}$ & $\begin{array}{l}\text { Depth } \\
\text { (mbsf) }\end{array}$ & Species $^{b}$ & $\delta^{18} \mathrm{O}$ & $\delta^{13} \mathrm{C}$ & $\begin{array}{l}\text { Sample } \\
\text { code }\end{array}$ & $\begin{array}{l}\text { Depth }^{\mathrm{a}} \\
\text { (mbsf) }\end{array}$ & Species $^{b}$ & $\delta^{18} \mathrm{O}$ & $\delta^{13} \mathrm{C}$ \\
\hline \multicolumn{5}{|c|}{ Hole 677A (Cont.) } & \multicolumn{5}{|c|}{ Hole 677A (Cont.) } \\
\hline A $88 / 1412$ & 96.60 & 12 & 4.00 & -2.44 & A $88 / 1492$ & 104.10 & 10,12 & 3.90 & -2.01 \\
\hline A $88 / 1413$ & 96.70 & 3 & 3.15 & -0.40 & A $88 / 1493$ & 104.20 & 3 & 3.17 & -0.92 \\
\hline A $88 / 1414$ & 96.80 & 8,11 & 3.94 & -1.11 & A $88 / 1494$ & 104.30 & 3 & 3.03 & -1.08 \\
\hline A $88 / 1415$ & 96.91 & 3,5 & 3.20 & -0.41 & A $88 / 1618$ & 105.00 & 2,13 & 3.44 & -1.86 \\
\hline A $88 / 1416$ & 97.00 & 3,5 & 3.15 & -0.10 & A $88 / 1619$ & 105.10 & 2,8 & 3.47 & -2.19 \\
\hline A $88 / 1417$ & 97.10 & 3 & 3.07 & -0.69 & A $88 / 1620$ & 105.18 & 9,12 & 3.52 & -1.49 \\
\hline A $88 / 1418$ & 97.18 & $2,10,11$ & 3.81 & -1.40 & A $88 / 1641$ & 105.30 & $3,5,6$ & 2.67 & -0.56 \\
\hline A $88 / 1419$ & 97.30 & 3 & 2.95 & -0.25 & A $88 / 1643$ & 105.40 & 8,11 & 3.64 & -2.61 \\
\hline A $88 / 1420$ & 97.40 & 8 & 3.68 & -0.79 & A $88 / 1644$ & 105.50 & 2,8 & 3.34 & -1.53 \\
\hline A $88 / 1421$ & 97.50 & 8 & 3.40 & -0.89 & A $88 / 1645$ & 105.60 & 2,8 & 3.83 & -2.09 \\
\hline A $88 / 1422$ & 97.60 & 8 & 3.69 & -0.91 & A $88 / 1646$ & 105.70 & 2 & 3.73 & -1.95 \\
\hline A $88 / 1423$ & 97.70 & 8 & 3.50 & -0.94 & A $88 / 1647$ & 105.80 & $1,2,11$ & 4.12 & -1.82 \\
\hline A $88 / 1424$ & 97.80 & 8 & 3.63 & -0.97 & A $88 / 1648$ & 105.80 & 3 & 3.09 & -1.09 \\
\hline A $88 / 1425$ & 97.80 & 3 & 2.83 & -0.37 & A $88 / 1649$ & 105.90 & 3 & 3.29 & -0.77 \\
\hline A $88 / 1426$ & 97.90 & 8 & 3.63 & -0.98 & A $88 / 1650$ & 105.90 & 2 & 4.08 & -1.95 \\
\hline A $88 / 1427$ & 98.00 & 8 & 3.69 & -1.22 & A $88 / 1651$ & 106.00 & 3 & 3.29 & -0.92 \\
\hline A $88 / 1428$ & 98.10 & 8 & 3.83 & -1.14 & A $88 / 1652$ & 106.10 & 3 & 3.22 & -0.84 \\
\hline A $88 / 1429$ & 98.20 & 8 & 4.04 & -1.20 & A $88 / 1653$ & 106.20 & 3,5 & 3.22 & -0.66 \\
\hline A $88 / 1430$ & 98.20 & 3 & 3.13 & -0.41 & A $88 / 1654$ & 106.20 & 1,2 & 4.09 & -1.99 \\
\hline A $88 / 1431$ & 98.30 & 1 & 4.38 & -1.38 & A $88 / 1655$ & 106.30 & 3 & 3.11 & -0.68 \\
\hline A $88 / 1432$ & 98.41 & 1 & 4.49 & -1.47 & A $88 / 1656$ & 106.37 & 12 & 4.11 & -2.44 \\
\hline A $88 / 1433$ & 98.50 & 1 & 4.47 & -1.45 & A $88 / 1657$ & 106.50 & 3 & 3.10 & -0.24 \\
\hline A $88 / 1434$ & 98.61 & 1 & 4.39 & -1.62 & A $88 / 1658$ & 106.60 & 1,2 & 3.62 & -1.39 \\
\hline A $88 / 1435$ & 98.61 & 3 & 3.65 & -0.65 & A $88 / 1659$ & 106.68 & 3 & 2.88 & -0.26 \\
\hline A $88 / 1436$ & 98.68 & 1 & 4.53 & -1.51 & A $88 / 1660$ & 106.79 & 3 & 3.08 & -0.57 \\
\hline A $88 / 1437$ & 98.80 & 2 & 4.27 & -2.18 & & & & & \\
\hline
\end{tabular}

Hole $677 \mathrm{~B}$

$\begin{array}{lllll}\text { A } 88 / 1437 & 98.80 & 2 & 4.27 & -2.18 \\ \text { A } 88 / 1438 & 98.90 & 3 & 3.49 & -0.80\end{array}$

$\begin{array}{lllll}\text { A } 88 / 1438 & 98.90 & 3 & 3.49 & -0.80 \\ \text { A } 88 / 1439 & 99.00 & 1 & 4.39 & -1.56\end{array}$

$\begin{array}{lllll}\text { A } 88 / 1439 & 99.00 & 1 & 4.39 & -1.56 \\ \text { A } 88 / 1440 & 99.00 & 3 & 3.43 & -0.84\end{array}$

$\begin{array}{lllll}\text { A } 88 / 1440 & 99.00 & 3 & 3.43 & -0.84 \\ \text { A } 88 / 1441 & 99.10 & 3 & 3.54 & -1.31 \\ \text { A } 88 / 1442 & 99.20 & 3 & 3.22 & -0.76\end{array}$

$\begin{array}{lllll}\text { A } 88 / 1442 & 99.20 & 3 & 3.22 & -0.76 \\ \text { A } 88 / 1443 & 99.30 & 3,5 & 3.14 & -0.90\end{array}$

$\begin{array}{lllll}\text { A } 88 / 1444 & 99.40 & 1,8 & 4.17 & -2.37\end{array}$

$\begin{array}{lllll}\text { A } 88 / 1445 & 99.50 & 3,5 & 3.31 & -0.65\end{array}$

$\begin{array}{lllll}\text { A } 88 / 1446 & 99.60 & 3.5 & 3.37 & -0.73\end{array}$

\begin{tabular}{lllll} 
A $88 / 1447$ & 99.70 & 2 & 3.37 & -0.73 \\
A $88 / 1448$ & 99.80 & 11 & 3.56 & -2.24 \\
\hline
\end{tabular}

$\begin{array}{lllll}\text { A } 88 / 1448 & 99.80 & 11 & 3.54 & -0.93 \\ \text { A } 88 / 1449 & 99.91 & 1,2,11 & 3.66 & -1.16\end{array}$

$\begin{array}{lrlrr}\text { A } 88 / 1449 & 99.91 & 1,2,11 & 3.66 & -1.16 \\ \text { A } 88 / 1450 & 100.00 & 2,11,12 & 3.52 & -1.40\end{array}$

$\begin{array}{lllll}\text { A } 88 / 1451 & 100.11 & 11,12 & 3.49 & -1.78\end{array}$

$\begin{array}{lllll}\text { A } 88 / 1452 & 100.18 & 9,11 & 3.52 & -0.68\end{array}$

$\begin{array}{lllll}\text { A } 88 / 1453 & 100.30 & 12 & 3.57 & -2.45\end{array}$

$\begin{array}{lllll}\text { A } 88 / 1454 & 100.40 & 2,8 & 3.58 & -1.91 \\ \text { A } 88 / 1455 & 100.50 & 2 & 3.32 & -1.83\end{array}$

$\begin{array}{lllll}\text { A } 88 / 1456 & 100.60 & 3 & 2.80 & -0.30\end{array}$

$\begin{array}{lllll}\text { A } 88 / 1457 & 100.70 & 2,8,10 & 3.48 & -1.78\end{array}$

$\begin{array}{lllll}\text { A } 88 / 1458 & 100.80 & 3 & 2.90 & -0.53\end{array}$

$\begin{array}{lllll}\text { A } 88 / 1459 & 100.90 & 3 & 3.28 & -0.56\end{array}$

$\begin{array}{lllll}\text { A } 88 / 1460 & 101.00 & 2 & 3.88 & -2.06 \\ \text { A } 88 / 1461 & 101.10 & 3 & 3.54 & -0.71\end{array}$

$\begin{array}{lllll}\text { A } 88 / 1462 & 101.20 & 1 & 4.45 & -1.79\end{array}$

$\begin{array}{lllll}\text { A } 88 / 1463 & 101.30 & 1 & 4.56 & -1.71\end{array}$

$\begin{array}{lllll}\text { A } 88 / 1464 & 101.31 & 2,8 & 3.56 & -2.01\end{array}$

$\begin{array}{lllll}\text { A } 88 / 1465 & 101.40 & 2,10,11 & 3.64 & -1.80\end{array}$

$\begin{array}{lllll}\text { A } 88 / 1466 & 101.50 & 1,2,8 & 3.72 & -1.62\end{array}$

$\begin{array}{lllll}\text { A } 88 / 1467 & 101.60 & 1 & 4.22 & -2.00 \\ \text { A } 88 / 1468 & 101.70 & 1 & 4.49 & -1.86\end{array}$

$\begin{array}{lllll}\text { A } 88 / 1468 & 101.70 & 1 & 4.49 & -1.86 \\ \text { A } 88 / 1469 & 101.70 & 3 & 3.47 & -1.04\end{array}$

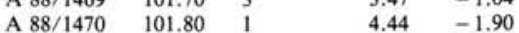

$\begin{array}{lllll}\text { A } 88 / 1471 & 101.91 & 1 & 4.39 & -1.92\end{array}$

A $88 / 1472 \quad 102.02 \quad 1 \quad 4.41 \quad-1.89$

$\begin{array}{lllll}\text { A } 88 / 1473 & 102.10 & 1 & 4.44 & -1.75\end{array}$

$\begin{array}{lllll}\text { A } 88 / 1474 & 102.18 & 1 & 4.40 & -1.85 \\ \text { A } 88 / 1475 & 102.30 & 1 & 4.36 & -1.82\end{array}$

$\begin{array}{lllll}\text { A } 88 / 1476 & 102.40 & 3 & 3.30 & -1.82\end{array}$

$\begin{array}{lllll}\text { A } 88 / 1477 & 102.50 & 3.5 & 3.29 & -0.95\end{array}$

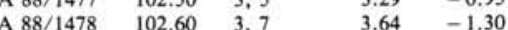

$\begin{array}{lllll}\text { A } 88 / 1479 & 102.70 & 12 & 4.23 & -1.99\end{array}$

$\begin{array}{lllll}\text { A } 88 / 1480 & 102.80 & 12 & 4.21 & -1.99\end{array}$

$\begin{array}{lllll}\text { A } 88 / 1481 & 103.00 & 1,2 & 3.61 & -1.80 \\ \text { A } 88 / 1482 & 103.10 & 1 & 4.53 & -1.89\end{array}$

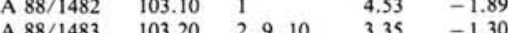

$\begin{array}{lllll}\text { A } 88 / 1483 & 103.20 & 2,9,10 & 3.35 & -1.30 \\ \text { A } 88 / 1484 & 103.30 & 5,6.7 & 2.79 & -0.58\end{array}$

$\begin{array}{lllll}\text { A } 88 / 1485 & 103.38 & 3,6 & 2.63 & -0.24\end{array}$

$\begin{array}{lllll}\text { A } 88 / 1486 & 103.50 & 2,10,12 & 3.44 & -1.89\end{array}$

$\begin{array}{lllll}\text { A } 88 / 1487 & 103.60 & 10.12 & 3.53 & -1.89\end{array}$

$\begin{array}{lllll}\text { A } 88 / 1488 & 103.68 & 13 & 3.63 & -0.70\end{array}$

$\begin{array}{lllll}\text { A } 88 / 1489 & 103.80 & 3 & 3.08 & -0.48\end{array}$

$\begin{array}{lllll}\text { A } 88 / 1490 & 103.90 & 5 & 3.20 & -0.79 \\ \text { A } 88 / 1491 & 104.00 & 2,9,10 & 3.73 & -1.20\end{array}$

\begin{tabular}{llllr} 
A $88 / 707$ & 5.48 & 1 & 4.28 & -1.11 \\
A 88/706 & 5.60 & 1 & 4.88 & -1.21 \\
A 88/705 & 5.70 & 1 & 4.76 & -1.17 \\
A 88/704 & 5.80 & 1 & 5.02 & -1.27 \\
A 88/703 & 5.90 & 1 & 5.10 & -1.32 \\
A 88/702 & 6.00 & 1 & 5.08 & -1.40 \\
A 88/701 & 6.10 & 5 & 4.26 & -0.81 \\
A 88/771 & 6.20 & 1,2 & 4.84 & -1.48 \\
A 88/772 & 6.20 & 4 & 5.46 & 1.18 \\
A 88/660 & 6.30 & 1,2 & 4.89 & -1.02 \\
A 88/659 & 6.40 & 1,2 & 4.89 & -1.48 \\
A 88/658 & 6.50 & 1 & 4.87 & -1.40 \\
A 88/657 & 6.60 & 1 & 4.95 & -1.43 \\
A 88/656 & 6.70 & 1 & 4.78 & -1.56 \\
A 88/655 & 6.80 & 1,2 & 4.66 & -1.62 \\
A 88/654 & 6.90 & 1 & 4.92 & -1.36 \\
A 88/653 & 6.98 & 1 & 4.82 & -1.51 \\
A 88/652 & 7.10 & 1 & 4.72 & -1.50 \\
A 88/651 & 7.20 & 1 & 4.69 & -1.45 \\
A 88/650 & 7.30 & 1 & 4.60 & -1.36 \\
A 88/634 & 14.60 & 1,2 & 4.68 & -1.64 \\
A 88/635 & 14.70 & 1,2 & 4.45 & -1.88 \\
A 88/636 & 14.80 & 1 & 4.41 & -1.40 \\
A 88/637 & 14.90 & 1 & 4.36 & -1.36 \\
A 88/638 & 15.00 & 1 & 4.34 & -1.28 \\
A 888/639 & 15.10 & 1 & 4.39 & -1.28 \\
A 88/640 & 15.20 & 1 & 4.39 & -1.31 \\
A 88/641 & 15.30 & 1 & 4.25 & -1.23 \\
A 88/642 & 15.40 & 1 & 4.27 & -1.21 \\
A 88/643 & 15.50 & 1 & 4.21 & -1.28 \\
A 88/644 & 15.60 & 1 & 4.09 & -1.06 \\
A 88/645 & 15.70 & 1 & 4.00 & -1.02 \\
A 88/646 & 15.78 & 1,2 & 3.81 & -1.12 \\
A 88/647 & 15.90 & 1 & 3.59 & -0.93 \\
A 88/648 & 16.00 & 1 & 3.81 & -0.77 \\
A 88/649 & 16.08 & 1 & 3.41 & -1.06 \\
A 88/708 & 24.50 & 1,2 & 4.12 & -1.39 \\
A 88/709 & 24.60 & 1 & 4.00 & -1.10 \\
A 88/710 & 24.70 & 1 & 4.00 & -1.11 \\
\hline 8 & & & & \\
\hline & & & & \\
\hline
\end{tabular}

a Depth from barrel sheets (Shipboard Scientific Party, 1988).

$\mathrm{b}_{1}=$ Uvigerina spp.; $2=$ Oridorsalis spp.; $3=$ Cibici doides wuellerstorfi; $4=$ Hoeglundina elegans; $5=$ Cibicidoides spp.; $6=$ Laticarinina pauperata; $7=$ Melonis spp.; 8 = Globocassidulina subglobosa; $9=$ Pyrgo spp.; 10 = Gyroidina spp.; 11 = Spaeroidina bulloides; $12=$ Globobulimina subglobosa; $13=S t i-$ lostomella sp.

${ }^{\mathrm{c}}$ Unreliable measurement or downhole contamination. 

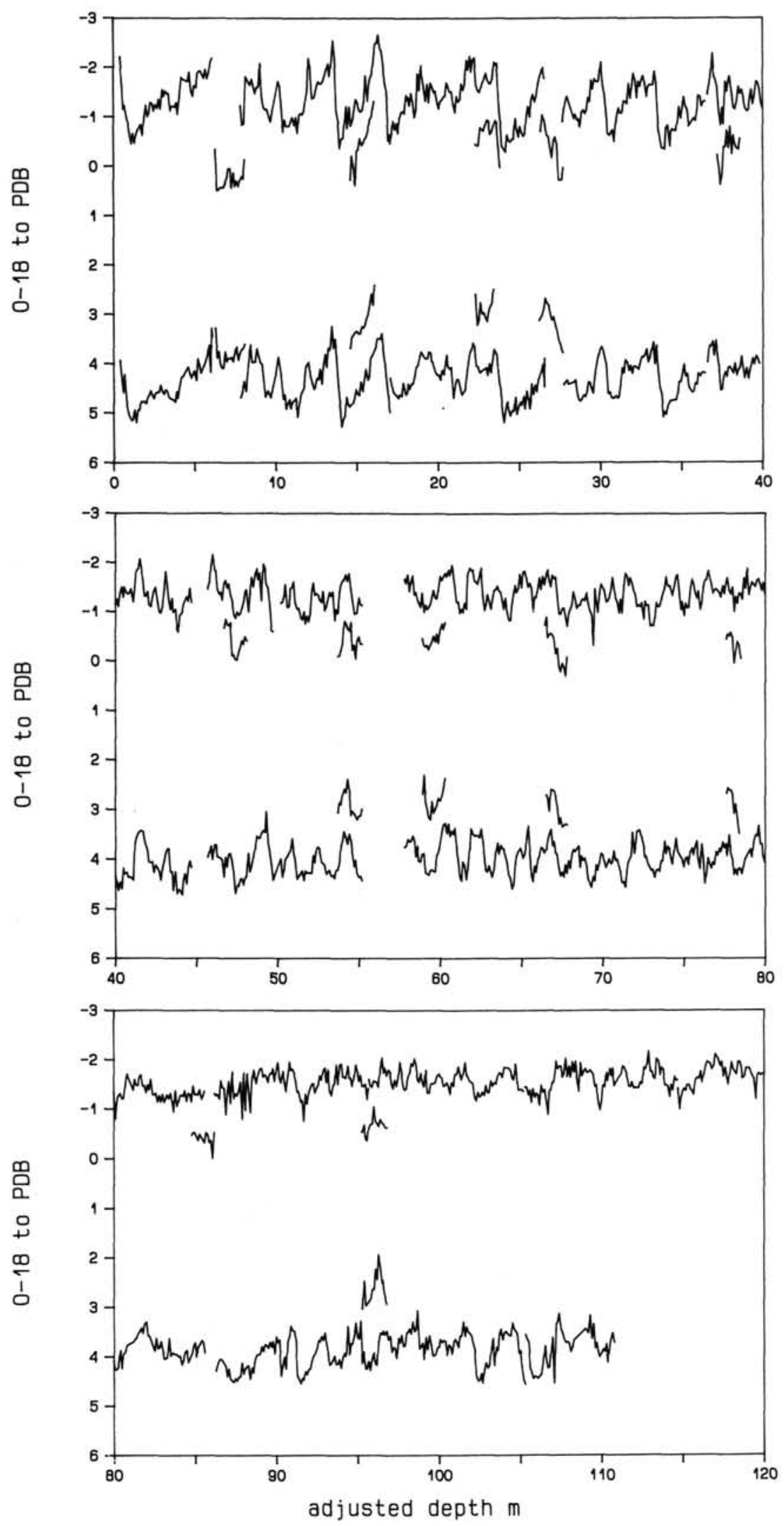

Figure 1. Oxygen isotope records for Globigerinoides ruber (upper track) and for benthic species adjusted to Uvigerina (lower track) in Hole 677A and (offset for clarity) in parts of Hole $677 \mathrm{~B}$, plotted for adjusted sub-bottom depths from Table 4. 
Table 4. Control points to generate combined depth sections.

\begin{tabular}{|c|c|c|c|}
\hline $\begin{array}{l}\text { Core, section, } \\
\text { interval }(\mathrm{cm})\end{array}$ & $\begin{array}{l}\text { Depth }^{a} \\
\text { (mbsf) }\end{array}$ & $\begin{array}{c}\text { Adjusted } \\
\text { depth from } \\
\text { Alexandrovich and } \\
\text { Hays (this volume) } \\
\text { (mbsf) }\end{array}$ & $\begin{array}{l}\text { Adjusted } \\
\text { depth from } \\
\text { this work } \\
\text { (mbsf) }\end{array}$ \\
\hline \multicolumn{4}{|l|}{ 111-677A- } \\
\hline $1 \mathrm{H}-1,40-42$ & 0.40 & 0.40 & 0.40 \\
\hline $1 \mathrm{H}-5,10-12$ & 6.10 & 6.10 & 6.10 \\
\hline $2 \mathrm{H}-1,29-41$ & 6.59 & 7.84 & 7.84 \\
\hline $2 \mathrm{H}-7,58-60$ & 15.78 & 17.03 & 17.03 \\
\hline $3 \mathrm{H}-1,10-12$ & 15.80 & 17.05 & 17.05 \\
\hline $3 \mathrm{H}-7,59-61$ & 25.29 & 26.54 & 26.54 \\
\hline $4 \mathrm{H}-1,8-10$ & 25.28 & 26.53 & 27.63 \\
\hline $4 \mathrm{H}-6,140-142$ & 34.10 & 35.35 & 36.45 \\
\hline $5 \mathrm{H}-1,10-12$ & 35.80 & 35.40 & 36.50 \\
\hline $5 \mathrm{H}-7,30-32$ & 44.00 & 43.60 & 44.70 \\
\hline $6 \mathrm{H}-1,10-12$ & 44.30 & 44.60 & 45.70 \\
\hline $6 \mathrm{H}-7,60-62$ & 53.79 & 54.09 & 55.19 \\
\hline $7 \mathrm{H}-1,10-12$ & 53.80 & 55.00 & 57.80 \\
\hline $7 \mathrm{H}-7,48-50$ & 63.18 & 64.38 & 67.18 \\
\hline $8 \mathrm{H}-1,10-12$ & 63.30 & 64.50 & 67.30 \\
\hline $8 \mathrm{H}-7,30-32$ & 72.50 & 73.70 & 76.50 \\
\hline $9 \mathrm{H}-1,0-2$ & 72.70 & 73.90 & 76.70 \\
\hline $9 \mathrm{H}-6,140-142$ & 81.60 & 82.80 & 85.60 \\
\hline $10 \mathrm{H}-1,0-2$ & 82.20 & 83.40 & 86.20 \\
\hline $10 \mathrm{H}-7,50-52$ & 91.70 & 92.90 & 95.70 \\
\hline $11 \mathrm{H}-1,0-2$ & 91.72 & 92.92 & 95.72 \\
\hline $11 \mathrm{H}-7,60-62$ & 101.30 & 102.50 & 105.30 \\
\hline $12 \mathrm{H}-1,0-2$ & 101.20 & 102.40 & 105.20 \\
\hline $12 \mathrm{H}-7,60-62$ & 110.80 & 112.00 & 114.80 \\
\hline $13 \mathrm{H}-1,1-3$ & 110.71 & 111.91 & 114.71 \\
\hline $13 \mathrm{H}-7,50-52$ & 120.20 & 121.40 & 124.20 \\
\hline $14 \mathrm{H}-1,10-12$ & 120.30 & 121.50 & 124.30 \\
\hline \multicolumn{4}{|l|}{ 111-677B- } \\
\hline $1 \mathrm{H}-4,98-100$ & 5.48 & 5.98 & 6.28 \\
\hline $1 \mathrm{H}-5,130-132$ & 7.30 & 7.80 & 8.10 \\
\hline $2 \mathrm{H}-5,100-102$ & 14.60 & 15.10 & 14.60 \\
\hline $2 \mathrm{H}-6,98-100$ & 16.08 & 16.58 & 16.08 \\
\hline $3 \mathrm{H}-3,50-52$ & 20.60 & 22.60 & 22.30 \\
\hline $3 \mathrm{H}-6,140-142$ & 26.00 & 28.00 & 27.70 \\
\hline $4 \mathrm{H}-6,0-2$ & 34.10 & 36.10 & 37.20 \\
\hline $4 \mathrm{H}-6,140-142$ & 35.50 & 37.50 & 38.60 \\
\hline $5 \mathrm{H}-6,0-2$ & 43.60 & 45.60 & 46.70 \\
\hline $5 \mathrm{H}-6,140-142$ & 45.00 & 47.00 & 48.10 \\
\hline $6 \mathrm{H}-2,80-82$ & 47.90 & 50.80 & 53.70 \\
\hline $6 \mathrm{H}-6,140-142$ & 54.90 & 57.80 & 60.70 \\
\hline $7 \mathrm{H}-6,1-3$ & 62.61 & 63.21 & 66.41 \\
\hline $7 \mathrm{H}-6,140-142$ & 64.00 & 64.60 & 67.80 \\
\hline $8 \mathrm{H}-6,1-3$ & 72.11 & 73.21 & 77.61 \\
\hline $8 \mathrm{H}-6,90-92$ & 73.00 & 74.10 & 78.50 \\
\hline $9 \mathrm{H}-6,0-2$ & 81.60 & 81.50 & 84.80 \\
\hline $9 \mathrm{H}-6,140-142$ & 83.00 & 82.90 & 86.20 \\
\hline $10 \mathrm{H}-6,0-2$ & 91.10 & 92.00 & 95.30 \\
\hline \multirow[t]{2}{*}{$10 \mathrm{H}-6,150-152$} & 92.60 & 93.50 & 96.80 \\
\hline & & $\begin{array}{c}\text { Adjusted depth } \\
\text { (mbsf) }\end{array}$ & \\
\hline
\end{tabular}

69-504-

$\begin{array}{lll}1-1,10 & 10.1 & 7.8 \\ 1-3,100 & 14.0 & 11.7 \\ 2-1,10 & 14.5 & 11.55 \\ 2-3,80 & 18.2 & 15.25 \\ 3-1,10 & 18.9 & 16.2 \\ 3-3,120 & 23.0 & 20.3 \\ 4-1,10 & 23.3 & 20.8 \\ 4-3,110 & 27.3 & 24.8 \\ 5-1,40 & 28.0 & 26.44 \\ 5-3,40 & 31.0 & 29.44 \\ 6-1,30 & 32.3 & 31.2 \\ 6-3,120 & 36.2 & 35.1 \\ 7-1,10 & 36.5 & 36.3 \\ 7-3,150 & 40.8 & 40.0 \\ 8-1,20 & 41.0 & 39.3 \\ 8-3,120 & 45.0 & 43.9\end{array}$

Table 4 (continued).

\begin{tabular}{llll}
\hline $\begin{array}{c}\text { Core, section, } \\
\text { interval (cm) }\end{array}$ & $\begin{array}{c}\text { Depth } \\
\text { (mbs) }\end{array}$ & $\begin{array}{c}\text { Adjusted depth } \\
\text { (mbsf) }\end{array}$ & $\begin{array}{c}\text { Adjusted } \\
\text { depth from } \\
\text { this work } \\
\text { (mbsf) }\end{array}$ \\
\hline 69-504- (Cont.) & & & \\
& & & \\
$9-1,60$ & 45.8 & 44.6 & \\
$9-3,120$ & 49.4 & 48.2 & \\
$10-1,100$ & 50.6 & 49.0 & \\
$10-3,70$ & 53.3 & 51.7 & \\
$11-1,20$ & 54.2 & 54.3 & \\
$11-3,110$ & 58.2 & 58.3 & \\
$12-1,10$ & 58.5 & 59.2 & \\
$12-3,120$ & 62.6 & 63.3 & \\
$13-1,10$ & 62.9 & 64.2 & \\
$13-3,80$ & 66.6 & 67.9 & \\
$14-1,90$ & 68.1 & 69.0 & 72.4 \\
$14-2,130$ & 71.5 & 73.7 & \\
$15-1,10$ & 71.7 & 82.0 & \\
$15-3,110$ & 80.0 & & \\
\hline
\end{tabular}

a Depth from barrel sheets (Shipboard Scientific Party, 1988).

Details of the patching are discussed in terms of successive cores in Hole 677A. Between Cores 111-677A-1H and 111$677 \mathrm{~A}-2 \mathrm{H}$ we have not yet analyzed sufficient material from Core 111-677B-1H to establish the exact overlap. Between Cores 111677A-2H and 111-677A-3H the only overlap is in Core 69-504$3 \mathrm{H}$. Between 111-677A-3H and 111-677A-4H the complete overlap is again only contained in $69-504-5 \mathrm{H}$ where we find the continuous transition from stage 17 to stage 18. Between Cores 111-677 A-4H and 111-677A-5H we adopt the estimate of Alexandrovich and Hays (this volume); we noted pipe rust in the top several samples from Core 111-677A-5H and agree with their judgement that this represents recored material. Between Cores $111-677 \mathrm{~A}-5 \mathrm{H}$ and $111-677 \mathrm{~A}-6 \mathrm{H}$ the situation is clear, particularly in view of the data from Hole 504. The relationship of Cores $111-677 \mathrm{~A}-6 \mathrm{H}$ and $111-677 \mathrm{~A}-7 \mathrm{H}$ is problematic. Alexandrovich and Hays (this volume) concluded that there is no material missing at this break, but were then forced to conclude that Cores 111-677B-6H and 111-677B-7H overlap by about $2 \mathrm{~m}$ although they observed no evidence for disturbance in the top of Core 111-677B-7H. We have tentatively reached a different conclusion: that there is a 2-m gap between Cores $111-677 \mathrm{~A}-6 \mathrm{H}$ and $111-677 \mathrm{~A}-7 \mathrm{H}$. This can only be confirmed by further work in Hole 677B, but corroboration comes from the record of Core 69-504-11 H, which does not correlate well with either the lower part of Core 111-677A-6H or with the upper part of Core 111677A-7H. Likewise, the overlaps between Cores 111-677A-7H through $111-677 \mathrm{~A}-10 \mathrm{H}$ can be confirmed only by further analyses in Hole 677B.

\section{Correlations}

Below oxygen isotope stage 22 few features of the oxygen isotope record are familiar. An important preliminary to developing a standard stratigraphy for the oxygen isotope record was to obtain key biostratigraphic information. On Figure 2 the biostratigraphic datum levels that establish the basis for extending the stratigraphy of the sequence are indicated, with their depths and published age estimates listed in Table 5. The published age estimates for four of these events are most closely linked with the $1.66 \mathrm{Ma}$ age (as assigned by Berggren et al., 1985) of the top of the Olduvai Chron, thus establishing that the stratigraphic position of this magnetic reversal boundary would be at about $73 \mathrm{~m}$ (adjusted sub-bottom depth) if it were recognizable in Hole 677A. Ruddiman et al. (1986) placed this boundary in stage 64 in their scheme. Comparison of their data and that of 

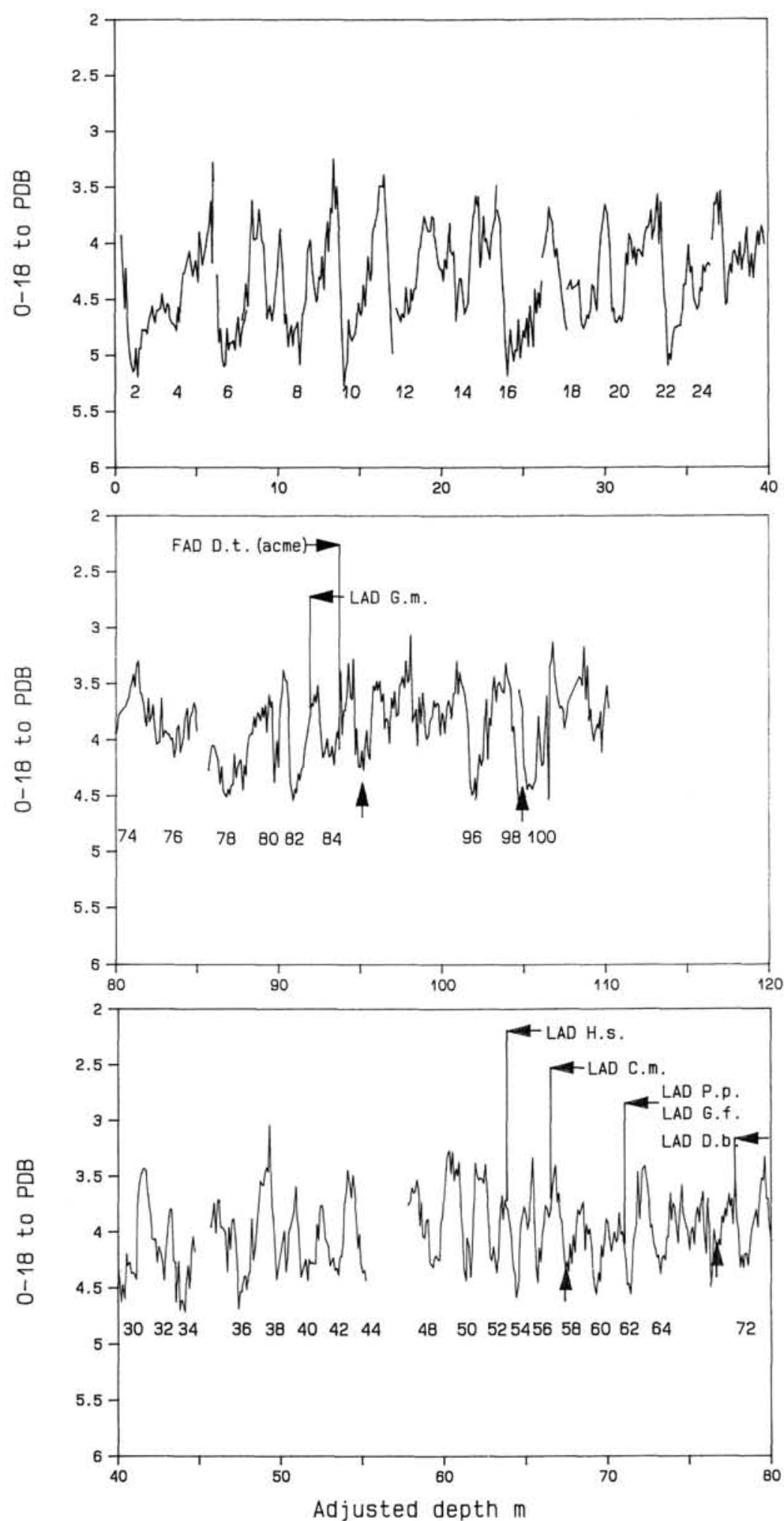

Figure 2. Oxygen isotope records for benthic species (adjusted to Uvigerina) plotted for adjusted sub-bottom depths from Table 4. Data from Hole 677A with patches from Cores 111-677B-1H and 111-677B-3H for the intercore gaps in Hole 677A at about 7 and $27 \mathrm{mbsf}$. Biostratigraphic levels from Table 5: LAD H.s. = last-appearance datum Helicosphaera sellii; LAD C.m. = LAD Calcidiscus macintyrei; LAD G.f. = LAD Globigerinoides fistulosus; LAD P.p. = LAD Pterocanium prismatium; LAD D.b. = LAD Discoaster brouweri; FAD D.t. (acme) $=$ base of Discoaster triradiatus acme; LAD G.m. = LAD Globorotalia miocenica. For clarity, only the even-numbered isotope stage numbers are labeled where clearly identified. Arrows pointing up indicate positions of intercore gaps where sediment is possibly missing. 
Table 5. Selected biostratigraphic datums in Hole 677A.

\begin{tabular}{lllllc}
\hline & \multicolumn{2}{c}{ Depth } & & \multirow{2}{*}{ Age } \\
\cline { 2 - 3 } \cline { 5 - 6 } \multicolumn{1}{c}{ Datum $^{\mathrm{a}}$} & $(\mathrm{mbsf})$ & Reference $^{\mathrm{b}}$ & & $(\mathrm{Ma})$ & Reference $^{\mathrm{b}}$ \\
\hline LAD Helicosphaera sellii & 59.8 & 1 & 1.37 & 3 \\
LAD Calcidiscus macintyrei & 62.5 & 1 & 1.47 & 3 \\
LAD Globigerinoides fistulosus & 66.8 & 2 & 1.6 & 4 \\
LAD Pterocanium prismatium & 66.75 & 5 & 1.6 & 4 \\
LAD Discoaster brouweri & 73.9 & 6 & 1.88 & 3 \\
FAD Discoaster triradiatus acme & 90.5 & 6 & 2.03 & 3 \\
LAD Globorotalia miocenica & 88.0 & 2 & 2.0 & 3 \\
\hline
\end{tabular}

${ }^{a}$ FAD $=$ first-appearance datum; LAD = last-appearance datum.

$\mathrm{b}_{1}=\mathrm{J}$. Backman (pers. comm., 1988); 2 = N.J.S. (this work); $3=$ Backman and Shackleton (1983); 4 = Berggren et al. (1980); $5=$ Alexandrovich (this volume); 6 = A. Chepstow-Lusty (pers, comm., 1988).

Hole 677A shows excellent agreement only if it assumed either that stages 45 and 46 are missing at a coring gap between Core $111-677 \mathrm{~A}-6 \mathrm{H}$ and $111-677 \mathrm{~A}-7 \mathrm{H}$ or that stages 56 and 57 are missing in a coring gap between Cores 111-677A-7H and 111$677 \mathrm{~A}-8 \mathrm{H}$. (It should be remarked that although the record of DSDP Site 607 is slightly inferior to that of Hole 677A in terms of sampling density, its validity is strongly supported by the accompanying $\mathrm{CaCO}_{3}$ data from both Site 607 and nearby Site 609; Ruddiman et al., 1986.) As discussed previously, we have tentatively concluded that the gap is between Cores 111-677A$6 \mathrm{H}$ and $111-677 \mathrm{~A}-7 \mathrm{H}$, and the isotope stages shown on Figure 2 are identified on this basis.

Working in the section of Site 607 below stage 64, Raymo et al. (in press) recovered an oxygen isotope record that is closely comparable with that shown here for benthic species in Hole $677 \mathrm{~A}$. The oxygen isotope stage numbering scheme used here was developed to be consistent with that for Site 607. Important features of the Site 607 record are as follows (Raymo et al., in press):

1. The base of the Olduvai and the last-appearance datum of Discoaster brouweri are located in stage 72.

2. Stages 78 and 82 represent relatively intense glacials, whereas stage 80 is a short, less intense glacial event. The base of the acme of the triradiate form of $D$. brouweri is found in stage 82 .

3. Stages 96,98 , and 100 represent intense glacials, with the first major glacial ice-rafting recognized off Britain at DSDP Site 552 in stage 100 (Shackleton et al., 1984).

4. The Gauss/Matuyama boundary is found in stage 104 .

Backman and Shackleton (1983) inferred that there was a hiatus in Site 504 spanning the interval 1.8-2.0 Ma, based on the absence of most of the interval in which the Discoaster population is characterized by the presence of up to $20 \%$ of the triradiate form Discoaster triradiatus. However, A. Chepstow-Lusty (pers. comm., 1988) showed that despite the surprisingly low abundance of Discoaster spp., both the interval with relatively abundant triradiate specimens of $D$. brouweri starting at about $92 \mathrm{~m}$ (adjusted sub-bottom depth) and the last-appearance datum of D. brouweri at about $77.5 \mathrm{~m}$ (adjusted sub-bottom depth) can be determined in Hole 677A. Thus, there is not a hiatus in this part of the column at either Site 677 or Hole 504. This enables us to identify stages 72 to 82 and stages 96 to 100 without difficulty. Definitive correlations for stages 65 to 70 and for stages 83 to 95 require first that we establish continuity between Cores 111-677A-8H and 111-677A-9H and between Cores $111-677 \mathrm{~A}-10 \mathrm{H}$ and $111-677 \mathrm{~A}-11 \mathrm{H}$.

Shackleton and Opdyke (1976) did not attempt to number stages below stage 23 , which was renumbered to stage 25 by
Ruddiman et al. (1986), because it did not appear at that time that an extension could be devised that would be both generally applicable and demonstrably reliable in a stratigraphic sense. This philosophy has appeared justifiable in that although van Donk (1976), Grazzini et al. (1983), and recently Williams et al. (1988) used extended numbering schemes, none of these has found general favor, nor did any of these workers attempt to use a previously proposed scheme. Ruddiman et al. (1986) demonstrated convincingly that the oxygen isotope record they obtained in Site 607 and the percent calcium carbonate records that they obtained from Sites 607 and 609 were both extensively dominated by 41,000 -yr cycles. They proposed a numbering scheme that they firmly linked to the underlying 41,000 -yr cycle. The earlier stages numbered here following Raymo et al. (in press) in Site 607 are also apparently associated with 41,000-yr cycles and have a characteristic wavelength in Site 677 sediments of about $1.5 \mathrm{~m}$ (although in both Sites 677 and 607 stage 78 appears too brief and too close to stage 80 to represent one of a regular series of $41,000-y r$ cycles). It is evident that these numbered oxygen isotope stages do have validity as a means of stratigraphic correlation between those sequences with adequate stratigraphic resolution for the stages to be identified.

The record of Hole $677 \mathrm{~A}$ has a significantly higher stratigraphic resolution than that of Site 607 , which should enable us to make a more reliable estimate of the extent of response to the precession cycle; this is a necessary preliminary to any meaningful modeling of the origin of the strikingly different character of the early Pleistocene record as compared with the last million years. In addition, the availability of two independent oxygen isotope records for planktonic and benthic foraminifers provides the opportunity for exploring the reliability of the record.

\section{Carbon Isotope Records}

Figure 3 shows the planktonic and benthic carbon isotope data on the same adjusted sub-bottom depth scale as Figure 2. Carbon isotope variability develops in a similar manner to oxygen isotope variability, with a poorly characterized record in the lower section, a very strong $41,000-\mathrm{yr}$ cyclic response to tilt variations in the middle section, and a record dominated by 100,000 -yr variability in the upper section. The Globigerinoides ruber ${ }^{13} \mathrm{C}$ record is noisy and probably not suitable for serious work on variations in atmospheric $\mathrm{CO}_{2}$ concentration. However, preliminary investigation shows that (1) throughout the record there is a general association between more negative $\delta^{13} \mathrm{C}$ values in benthic foraminifers and more positive $\delta^{18} \mathrm{O}$ values, as observed by Shackleton (1977) among others for the late Pleistocene; (2) the range of variation in $\delta^{13} \mathrm{C}$ is greater in $G$. ruber (planktonic) than in benthic species; and (3) this relationship implies that $\Delta \delta^{13} \mathrm{C}$ (the vertical ${ }^{13} \mathrm{C}$ gradient in the water column monitored as the isoiopic difference between planktonic and benthic specimens) was greater in interglacial times. This last observation would at first sight imply lower atmospheric carbon dioxide concentration in interglacials according to the model of Broecker (1982), contrary to observations for the late Pleistocene (Barnola et al., 1988; Shackleton and Pisias, 1985). However, particularly in the middle section where the cycles are clear, $\Delta \delta^{13} \mathrm{C}$ maxima (implying atmospheric $\mathrm{pCO}_{2}$ minima) may actually tend to fall on the interglacial/glacial transitions, which could be interpreted as extending the observation of Shackleton and Pisias (1985) that for the past $350,000 \mathrm{yr}$ changes in atmospheric carbon dioxide concentration may have been part of the forcing of climatic change. This question must be addressed with much more care before taking this as a firm conclusion. However, it is clear from these data that important variability in the ocean carbon system at orbital frequencies was a feature of the Pliocene-Pleistocene and not only of the most recent major glacial cycles. 

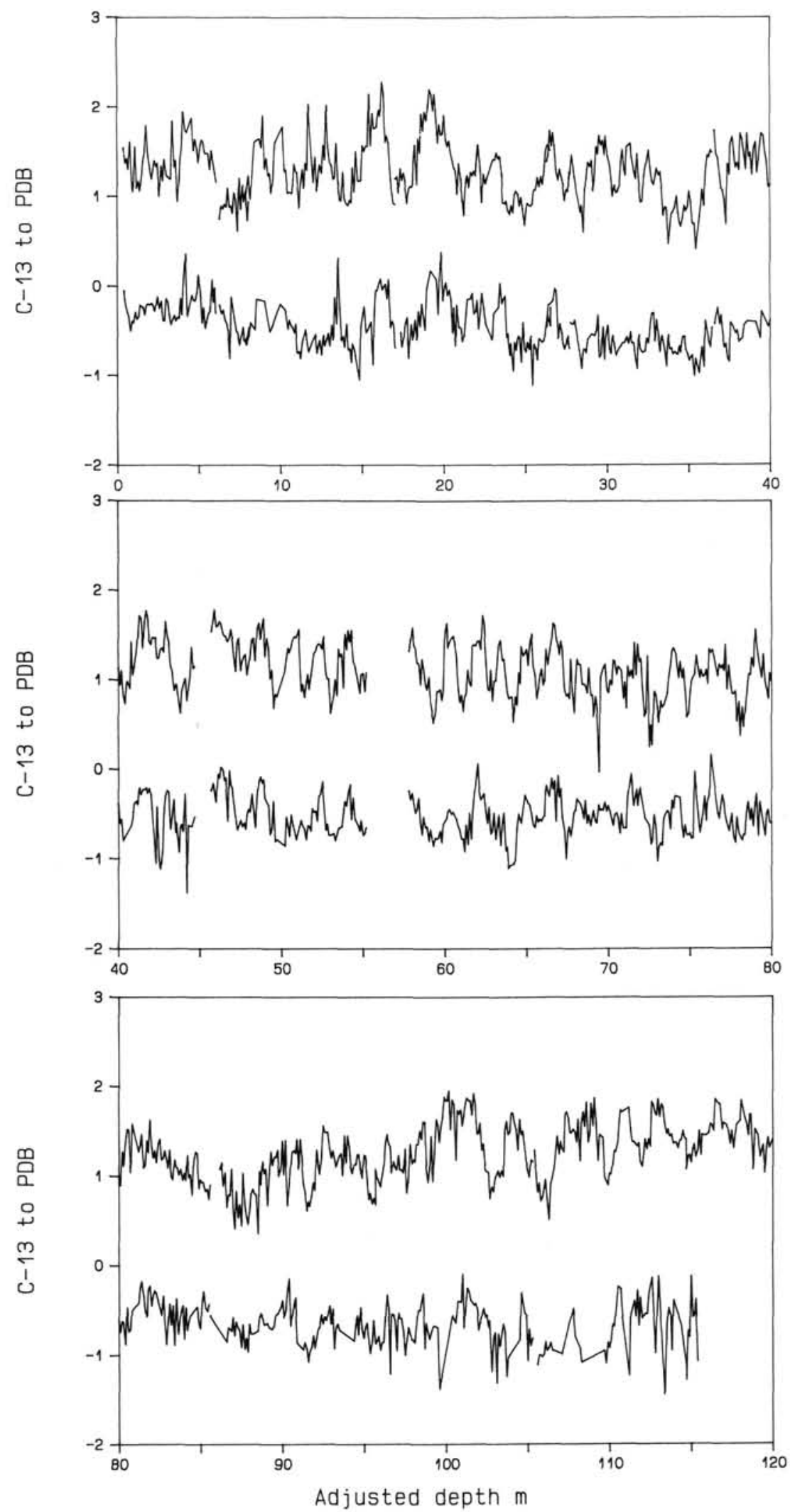

Figure 3. Carbon isotope records for Globigerinoides ruber (upper track) and benthic species adjusted to Cibicidoides (lower track) plotted for adjusted sub-bottom depth from Table 4. No data are plotted at levels for which uncalibrated benthic species or mixtures of species were analyzed. 


\section{CONCLUSIONS}

The planktonic and benthic oxygen isotope records of the entire Pleistocene at ODP Site 677 are significantly superior to any other Pacific data sets and will provide an excellent basis for an astronomically calibrated chronology for this interval. The oxygen isotope record is demonstrated for the first time to be of major value for stratigraphic correlation beyond the past million years; the isotope stages back to stage 104 that were documented by Ruddiman et al. (1986) and by Raymo et al. (in press) for the Atlantic are clearly recognizable in the Pacific.

\section{ACKNOWLEDGMENTS}

We thank the scientific crew of ODP Leg 111 for devoting the time to coring the sediment section at Site 677 when their sights were firmly set on the underlying basalts, as well as for the efforts that they gave in taking samples for this investigation. Julie Cartlidge has cheerfully undertaken the task of sieving all the samples as well as of picking the majority of the $G$. ruber for isotopic analysis. We would almost certainly have been unable to derive a correct correlation had Maureen Raymo not generously shared her unpublished data and interpretation of DSDP Site 607 material. This study was supported by NERC Grant GR3/3606.

\section{REFERENCES}

Backman, J., and Shackleton, N. J., 1983. Quantitative biochronology of Pliocene and early Pliocene calcareous nannofossils from the Atlantic, Indian and Pacific oceans. Marine Micropaleontol., 8:141170.

Barnola, J. M., Raynaud, D., Korotkevich, Y. S., and Lorius, C., 1987. Vostok ice core provides 160,000-year record of atmospheric $\mathrm{CO}_{2}$. Nature, 329:408-413.

Berggren, W. A., Burckle, L. H., Cita, M. B., Cooke, H.B.S., Funnell, B. M., Gartner, S., Hays, J. D., Kennett, J. P., Opdyke, N. D., Pastouret, L., Shackleton, N. J., and Takayanagi, Y., 1980. Towards a Quaternary timescale. Quat. Res. (N.Y.), 13:277-302.

Berggren, W. A., Kent, D. V., and Flynn, J. J., 1985. Jurassic to Paleogene: part 2. Paleogene chronology and chronostratigraphy. In Snelling, N. J. (Ed.), The Chronology of the Geological Record: Mem. Geol. Soc. (London), 10:141-186.

Broecker, W. S., 1982. Glacial to interglacial changes in ocean chemistry. Prog. Oceanogr., 11:151-197.

van Donk, J., 1976. An ${ }^{18} \mathrm{O}$ record of the Atlantic Ocean for the entire Pleistocene. In Cline, R. M., and Hays, J. D. (Eds.), Investigation of Late Quaternary Paleoclimatology: Geol. Soc. Am. Mem., 145: $147-164$.

Emiliani, C., 1955. Pleistocene temperatures. J. Geol., 63:538-578.

Epstein, S., Buchsbaum, S. R., Lowenstam, H. A., and Urey, H., 1953. Revised carbonate-water isotopic temperature scale. Geol. Soc. Am. Bull., 64:1315-1326.

Grazzini, C. V., Grably, M., Pujol, C., and Duprat, J., 1983. Oxygen isotope stratigraphy and paleoclimatology of southwestern Atlantic Quaternary sediments (Rio Grande Rise) at DSDP Site 517. In Barker, P. F., Carlson, R. L., Johnson, D. A., et al., Init. Repts. DSDP, 72: Washington (U.S. Govt. Printing Office), 871-884.

Prell, W. L., Gardner, J. V., et al., 1982. Leg 68: introduction, explanatory notes, and conventions. In Prell, W. L., Gardner, J. V., et al.,
Init. Repts. DSDP, 68: Washington (U.S. Govt. Printing Office), 513.

Raymo, M., Ruddiman, W. F., Backman, J., Clement, B. M., and Martinson, D. G., in press. Late Pliocene variation in Northern Hemisphere ice sheets and North Atlantic Deep Water circulation. Paleoceanography.

Ruddiman, W. F., Raymo, M., and McIntyre, A., 1986. Matuyama 41,000-years cycles: North Atlantic Ocean and Northern Hemisphere ice sheets. Earth Planet. Sci. Lett., 80:117-129.

Ruddiman, W. F., Raymo, M. E., Martinson, D. G., Clement, B. M., and Backman, J., in press. Pleistocene evolution of Northern Hemisphere climate. Paleoceanography.

Shackleton, N. J., 1977. Carbon-13 in Uvigerina: tropical rainforest history and the equatorial Pacific carbonate dissolution cycles. In Andersen, N. R. and Malahoff, A. (Eds.), The Fate of Fossil Fuel $\mathrm{CO}_{2}$ in the Oceans: New York (Plenum), 401-427.

Shackleton, N. J., and Hall, M. A., 1983. Stable isotope record of Hole 504 sediments: high-resolution record of the Pleistocene. In Cann, J. R., Langseth, M. G., Honnorez, J., Von Herzen, R. P., White, S. M., et al., Init. Repts. DSDP, 69: Washington (U.S. Govt. Printing Office), 431-441.

Shackleton, N. J., and Opdyke, N. D., 1973. Oxygen isotope and palaeomagnetic stratigraphy of equatorial Pacific Core V28-238: oxygen isotope temperatures and ice volumes on a $10^{5}$ year and $10^{6}$ year scale. Quat. Res. (N.Y.), 3:39-55.

1976. Oxygen isotope and palaeomagnetic stratigraphy of Pacific Core V28-239, late Pliocene to latest Pleistocene. In Cline, R. M., and Hays, J. D. (Eds.), Investigation of Late Quaternary Paleoceanography and Paleoclimatology: Mem. Geol. Soc. Am., 146: 449-464.

Shackleton, N. J., and Pisias, N. G., 1985. Atmospheric carbon dioxide, orbital forcing and climate. In Sundquist, E. D., and Broecker, W. S. (Eds.), The Carbon Cycle and Atmospheric $\mathrm{CO}_{2}:$ Natural Variations Archean to Present: Washington (Am. Geophys. Union), 303-317.

Shackleton, N. J., Backman, J., Zimmerman, H., Kent, D. V., Hall, M. A., Roberts, D. G., Schnitker, D., Baldauf, J. G., Despraires, A., Homrighausen, K., Huddlestun, P., Keene, J. B., Kaltenback, A. J., Krumsiek, K.A.O., Morton, A. C., Murray, J. W., and WestbergSmith, J., 1984. Oxygen isotope calibration of the onset of ice-rafting and history of glaciation in the North Atlantic region. Nature, 307:620-623.

Shipboard Scientific Parties of Leg 68 (Site 501), Leg 69, and Leg 70, 1983. Sites 501 and 504: sediments and ocean crust in an area of high heat flow on the southern flank of the Costa Rica Rift. In Cann, J. R., Langseth, M. G., Honnorez, J., Von Herzen, R. P., White, S. M., et al., Init. Repts. DSDP, 69: Washington (U.S. Govt. Printing Office), 31-173.

Shipboard Scientific Party, 1988. Sites 677 and 678. In Becker, K., Sakai, H., et al., Proc. ODP, Init. Repts., 111: College Station, TX (Ocean Drilling Program), 253-346.

Williams, D. F., Thunell, R. C., Tappa, E., Rio, D., and Raffi, I., 1988. Chronology of the Pleistocene oxygen isotope record: $0-1.8$ m.y. B.P. Palaeogeogr., Palaeoclimatol., Palaeoecol., 64:221-240.

Date of initial receipt: 14 November 1988

Date of acceptance: 2 June 1989

Ms 111B-150

Note: Analysis of additional samples from Hole 677B received in August 1989 shows that some of the depth adjustments in Table 4 are incorrect. The adjusted depth in Core 111-677 A-7H should be as proposed by Alexandrovich and Hays (this volume) and not as illustrated in Figures 1 through 3. Consequently, cold stages labeled 48 through 56 on Figure 2 should be stages 46 through 54 . 